\title{
Multirecycling of Plutonium from LMFBR Blanket in Standard PWRs Loaded with MOX Fuel
}

Sonat Sen Gilles Youinou

February 2013

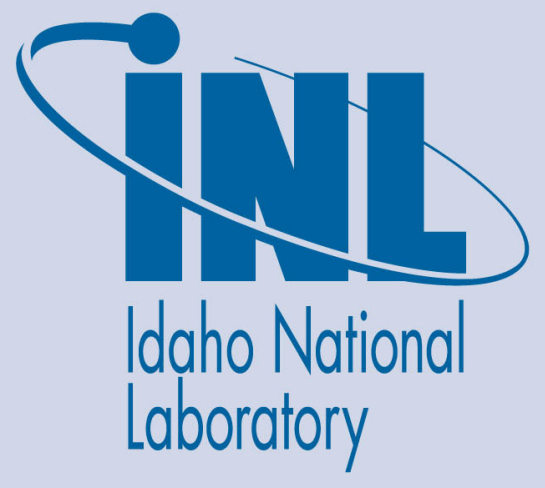

The INL is a U.S. Department of Energy National Laboratory operated by Battelle Energy Alliance 
INL/EXT-13-28448

FCRD-FCT-2013-000104

\title{
Multirecycling of Plutonium from LMFBR Blanket in Standard PWRs Loaded with MOX Fuel
}

\author{
Sonat Sen \\ Gilles Youinou
}

February 2013

\author{
Idaho National Laboratory \\ Fuel Cycle Research and Development \\ Idaho Falls, Idaho 83415
}

http://www.inl.gov

Prepared for the

U.S. Department of Energy

Office of Nuclear Energy

Under DOE Idaho Operations Office

Contract DE-AC07-05ID14517 


\section{DISCLAIMER}

This information was prepared as an account of work sponsored by an agency of the U.S. Government. Neither the U.S. Government nor any agency thereof, nor any of their employees, makes any warranty, expressed or implied, or assumes any legal liability or responsibility for the accuracy, completeness, or usefulness, of any information, apparatus, product, or process disclosed, or represents that its use would not infringe privately owned rights. References herein to any specific commercial product, process, or service by trade name, trade mark, manufacturer, or otherwise, does not necessarily constitute or imply its endorsement, recommendation, or favoring by the U.S. Government or any agency thereof. The views and opinions of authors expressed herein do not necessarily state or reflect those of the U.S. Government or any agency thereof. 
Multirecycling of Plutonium from LMFBR Blanket in Standard PWRS Loaded with MOX Fuel

February 2013 
Multirecycling of Plutonium from LMFBR Blanket in Standard PWRS Loaded with MOX Fuel 


\section{Multirecycling of Plutonium from LMFBR Blanket in Standard PWRS Loaded with MOX Fuel}

February 2013

\section{SUMMARY}

It is now well-known that, from a physics standpoint, $\mathrm{Pu}$, or even TRU (i.e. Pu+M.A.), originating from LEU fuel irradiated in PWRs can be multirecycled also in PWRs using MOX fuel. However, the degradation of the isotopic composition during irradiation necessitates using enriched $\mathrm{U}$ in conjunction with the MOX fuel either homogeneously or heterogeneously to maintain the $\mathrm{Pu}$ (or TRU) content at a level allowing safe operation of the reactor, i.e. below about $10 \%$.

The study is related to another possible utilization of the excess $\mathrm{Pu}$ produced in the blanket of a LMFBR, namely in a PWR(MOX). In this case the more Pu is bred in the LMFBR, the more PWR(MOX) it can sustain. The important difference between the Pu coming from the blanket of a LMFBR and that coming from a PWR(LEU) is its isotopic composition. The first one contains about $95 \%$ of fissile isotopes whereas the second one contains only about $65 \%$ of fissile isotopes. As it will be shown later, this difference allows the PWR fed by Pu from the LMFBR blanket to operate with natural $U$ instead of enriched $U$ when it is fed by Pu from PWR(LEU). 
Multirecycling of Plutonium from LMFBR Blanket in Standard PWRS Loaded with MOX Fuel 


\section{CONTENTS}

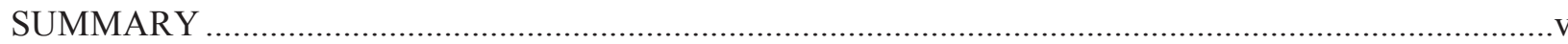

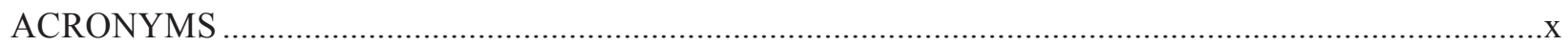

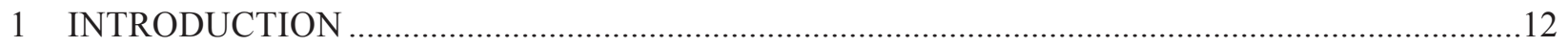

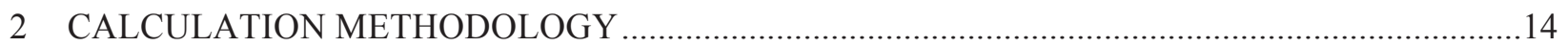

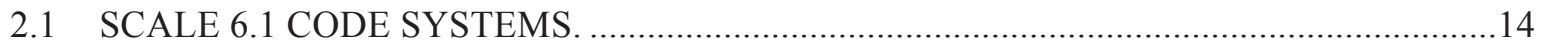

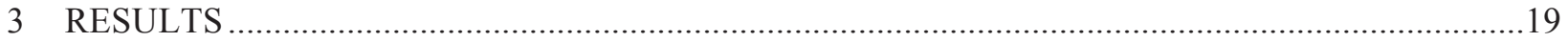

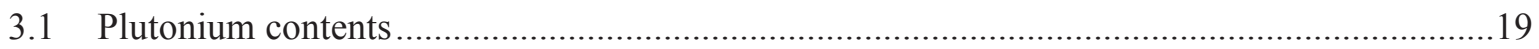

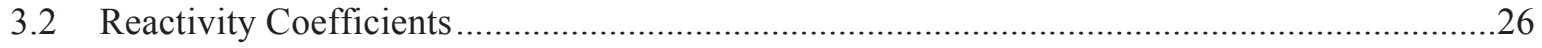

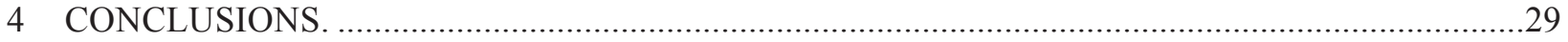

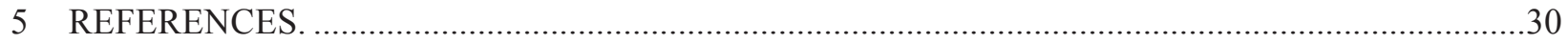

Appendix 1: Detailed isotopic mass fraction input (after 2-yrs fuel aging) ............................................31

Appendix 2: Detailed isotopic mass fraction output (discharged burn-up $50 \mathrm{GWd}$ / tHM)......................41

Appendix 3: Mid-cycle 1-group cross sections of most important actinides..........................................55

\section{FIGURES}

Figure 2-1: TRITON model (one fourth of a standard 17x17 fuel assembly ….................................... 15

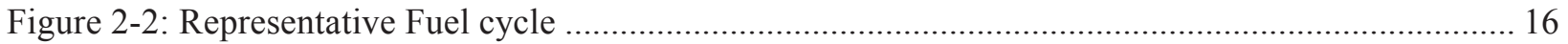

Figure 2-3: K-infinity trends vs. burnup showing the intersection point at $33.33 \mathrm{GWd} / \mathrm{tHM} \ldots \ldots \ldots \ldots \ldots \ldots \ldots . . . . .18$

Figure 3-1: $\mathrm{U}$ and Pu percentages in PWR MOX-NU fuel assemblies per cycle.................................... 20

Figure 3-2: Weight percent of Pu isotopes in PWR MOX-NU assemblies per cycle ............................. 20

Figure 3-3: Isotopic composition of Pu in PWR MOX-NU assemblies per cycle (BOL only)................. 21

Figure 3-4: Isotopic composition of Pu in PWR MOX-NU assemblies per cycle (BOL and EOL) .......... 22

Figure 3-5: Weight percentages of Pu in PWR MOX-NU assemblies per cycle .................................... 22

Figure 3-6: The origin of Pu in PWR MOX-NU assemblies per cycle ................................................. 23

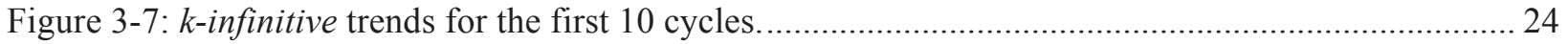

Figure 3-8: Fuel Temperature coefficient for the 15th Cycle …........................................................... 27

Figure 3-9: Moderator Temperature coefficient for the 15th Cycle ....................................................... 27

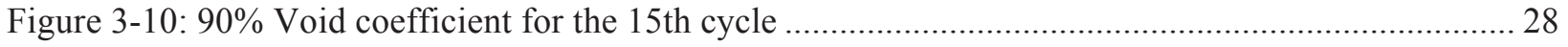




\section{TABLES}

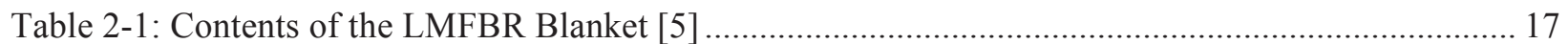

Table 3-1: Pu and U percentages in the fresh fuel per cycle................................................................. 19

Table 3-2: Mass Fraction input and output (summary) ....................................................................... 25

Table 3-3: Reactivity Coefficients calculated for the $15^{\text {th }}$ cycle ............................................................ 26 
Multirecycling of Plutonium from LMFBR Blanket in Standard PWRS Loaded with MOX Fuel

February 2013 


\section{ACRONYMS}

BOL Beginning of Life

EOL End of Life

GWd/tHM Gigawatt-days per tonne of initial heavy metal

INL Idaho National Laboratory

LEU Low-Enriched Uranium

LMFBR Liquid Metal Fast Breeder Reactor

MA Minor Actinide

MOX Mixed Oxide Fuel

MOX-NU Mixed Oxide Fuel with Natural Uranium Support

MOX-UE Mixed Oxide Fuel with Enriched Uranium Support

PWR Pressurized Water Reactor

TRU Transuranics 
Multirecycling of Plutonium from LMFBR Blanket in Standard PWRS Loaded with MOX Fuel

February 2013 


\section{MULTIRECYCLING OF PLUTONIUM FROM LMFBR BLANKET IN STANDARD PWRS LOADED WITH MOX FUEL}

\section{INTRODUCTION}

It is now well-known that, from a physics standpoint, $\mathrm{Pu}$, or even TRU (i.e. $\mathrm{Pu}+\mathrm{M}$.A.), originating from LEU fuel irradiated in PWRs can be multirecycled also in PWRs using MOX fuel. However, the degradation of the isotopic composition during irradiation necessitates using enriched $U$ in conjunction with the MOX fuel either homogeneously (MOX-UE approach [1]) or heterogeneously (CORAIL approach [2]) to maintain the Pu (or TRU) content at a level allowing safe operation of the reactor, i.e. below about $10 \%$.

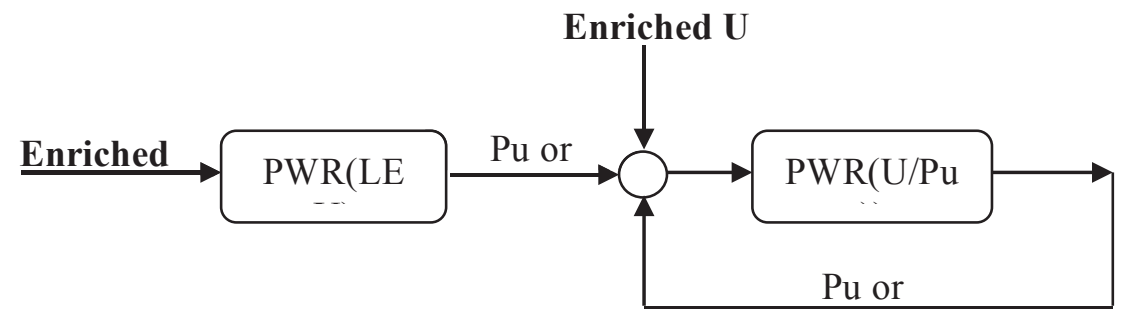

Figure 1-1. Schematic of the multirecycling of Pu or TRU originating from PWR(LEU) in PWR(MOX) using enriched U

It is also well-known that a LMFBR using $\mathrm{U}$ blankets can produce more $\mathrm{Pu}$ than it actually needs and that this $\mathrm{Pu}$ can be used to start another LMFBR. The more Pu it breeds, the less time it takes to have enough $\mathrm{Pu}$ to start a second LMFBR, i.e. the shorter the doubling time.

The results presented in this report are related to another possible utilization of the excess $\mathrm{Pu}$ produced in the blanket of a LMFBR, namely in a PWR(MOX). In this case the more Pu is bred in the LMFBR, the more PWR(MOX) it can sustain. The important difference between the Pu coming from the blanket of a LMFBR and that coming from a PWR(LEU) is its isotopic composition. The first one contains about $95 \%$ of fissile isotopes whereas the second one contains only about $65 \%$ of fissile isotopes. As it will be shown later, this difference allows the PWR fed by $\mathrm{Pu}$ from the LMFBR blanket to operate with natural $U$ instead of enriched $U$ when it is fed by $\mathrm{Pu}$ from PWR(LEU). 
Multirecycling of Plutonium from LMFBR Blanket in Standard PWRS Loaded with MOX Fuel

February 2013

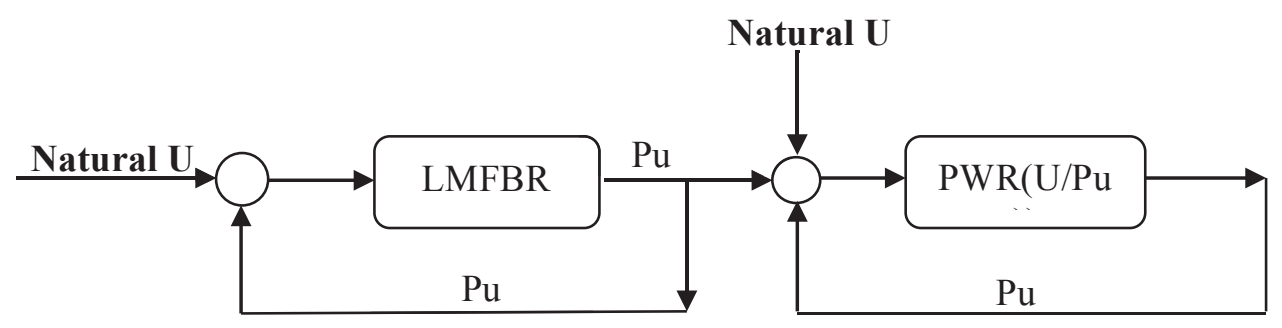

Figure 1-2. Schematic of the multirecycling of the excess Pu originating from LMFBR in PWR(MOX) using natural U 


\section{CALCULATION METHODOLOGY}

The calculations needed by this study have been performed through the code SCALE 6.1/TRITON. A small overview on the main capabilities and models used is reported in the following paragraphs.

\subsection{SCALE 6.1 CODE SYSTEMS.}

The reactor physics calculations have been performed by the lattice physics capabilities of the SCALE 6.1 code systems. The calculation flow consists of the use of several modules mutually coupled. The discrete-ordinates code NEWT (New ESC-based Weighting Transport code) coupled to the depletion code ORIGEN [3] via the TRITON control module [4]. Using the discrete-ordinates approximation to the transport equation on an arbitrary grid, together with a 238-group neutron cross-section library based on ENDF/B-VII, NEWT provides a robust and rigorous deterministic solution for non-orthogonal configurations. The differencing scheme employed by NEWT, the Extended Step Characteristic Approximation, allows a computational two-dimensional mesh based on arbitrary polygons. Such a mesh can be used to closely approximate curved or irregular surfaces to provide the capability to model problems that were formerly difficult or impractical to model directly with discrete-ordinates methods.

The TRITON control module performs the task of coordination of data transfer between various physics codes available within SCALE 6.1 and of invoking those codes in the proper sequence for a desired type of calculation. The high-fidelity nature of the NEWT solution in estimating angular flux distributions combined with the rigor of the ORIGEN depletion solver gives TRITON the capability to perform precise burnup-dependent physics calculations with few implicit approximations, and limited primarily by the accuracy of nuclide cross-sectional data. Such rigor may be necessary to capture the unique attributes of MOX-NU fuel behavior as well as that of advanced, highly heterogeneous fuel assembly designs being deployed in currentgeneration reactors. Cross-sectional self-shielding is carried out by BONAMI for unresolvedrange resonance data; the resolved resonance processor module CENTRM performs a 1-D discrete-ordinates code that uses point-wise cross-section data to produce a set of continuousenergy fluxes at discrete spatial intervals for each unit cell. Following a CENTRM calculation, the code PMC uses the resulting flux to collapse the point-wise continuous-energy cross sections into multi-group cross sections for each nuclide in each material in a unit (e.g., pin cell). The result is a multi-group library in which point cross-sectional data are weighted using the explicit point-wise spectrum representative of the nuclides present in a pin cell. Effects from overlapping resonances, fissile material in the fuel and surrounding moderator, anisotropic scattering, and inelastic level scattering are explicitly handled by this approach.

For the physics calculations carried out during this study, a TRITON model of one fourth standard (17x17) fuel assembly has been used (Figure 2-1). All the MOX-NU rods have the same Uranium and Plutonium content. The $0.5 \mathrm{~mm}$ water gap at the periphery is explicitly represented. The model uses three different burn-up zones to take into account the different local moderating 
ratios: 1 for the corner rods (green), 1 for the other rods located at the periphery (cyan) and 1 for the internal rods (red).

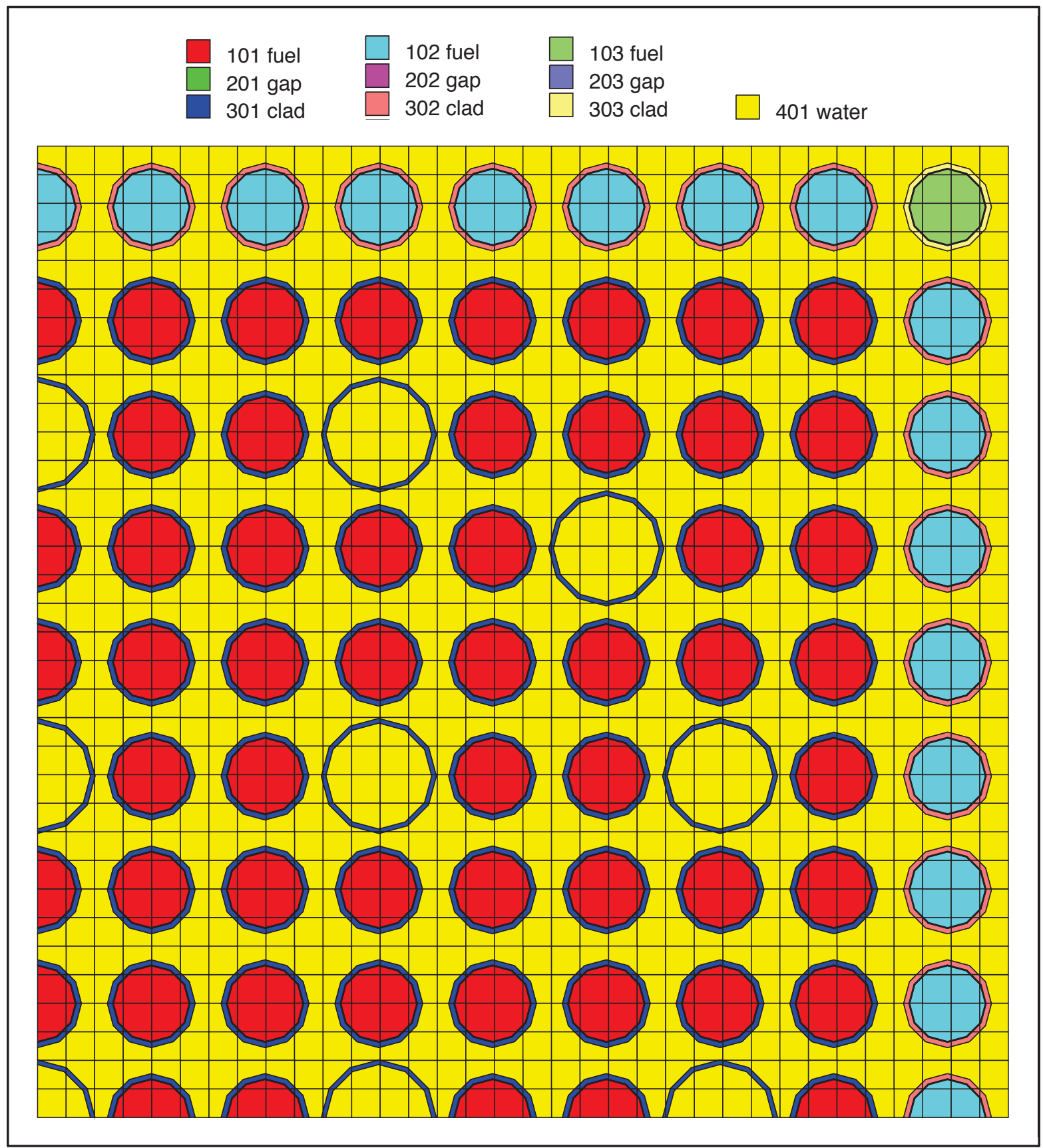

Figure 2-1: TRITON model (one fourth of a standard 17x17 fuel assembly

The calculations carried out to simulate the fuel cycle represented on the Figure 2-2. After each cycle, the amount of fissile Pu initially loaded that disappeared by either fission, capture or decay is replaced with $\mathrm{Pu}$ coming from LMFBR blanket with the amount required to achieve target burnup. The percentage of $\mathrm{Pu}$ coming from LMFBR blanket used in the manufacturing process decreases after the first cycle and reaches to an equilibrium. 


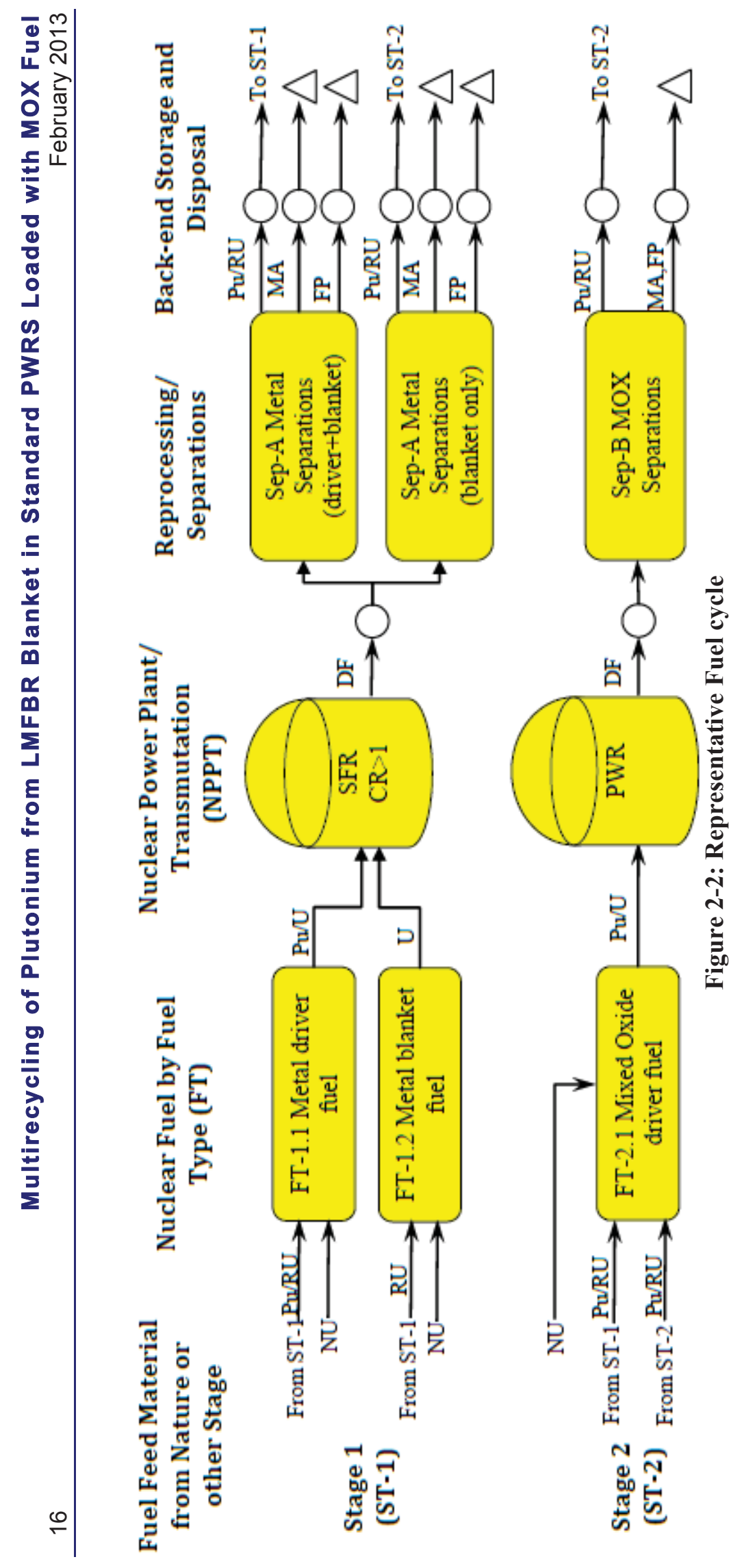


The $\mathrm{Pu}$ content in the MOX-NU fuel assemblies has been tuned to maintain the same average discharge burn-up and a constant cycle length. The calculations have been run considering a 3batch core with an average discharge burn-up of 50.0 GWd/tHM and a fuel power density of 35.36 MW/tHM. The model takes in account 2 year fuel aging (time needed by the fabrication and transport operations) and 5 years of cooling time (time needed in order to reduce the decay heat generation to levels compatible with the separation operations). The burn-up level above specified is reached after $\sim 1414$ days of irradiation. One cycle then corresponds to 2 years + $50 \mathrm{GWd} /$ tHM (1414 days) + 5 years cooling time $\cong 10.9$ years.

The necessary $P u$ enrichment are determined with a methodology that is standard for this kind of application: the k-infinity of the assembly at the average end of cycle burn-up (33.33 $\mathrm{GWd} / \mathrm{tHM}$ ), without soluble boron, is equal to that of the reference $4.2 \% \mathrm{UOX}$ assembly calculated using the same code (SCALE 6.1), methods (SN), nuclear data (238 group library based on ENDF/B - VII), etc., i.e. $\mathbf{k - i n f}=\mathbf{1 . 0 2 9 6 4 3}$ in our case. Figure 2-3, below, shows the kinfinity trends for the first 10 cycles as a function of burnup in $\mathrm{GWd} / \mathrm{tHM}$. The vertical line in Figure 2-3 corresponds to $33.33 \mathrm{GWd} / \mathrm{tHM}$, where k-infinity for all the cases is equal to each other.

MOX-NU density of $10 \mathrm{~g} / \mathrm{cm}^{3}$ is used throughout the analysis.

The isotopic percentages of the Pu coming from the LMFBR blanket are given in Table 2-1.

Table 2-1: Contents of the LMFBR Blanket [5]

\begin{tabular}{|l|r|}
\hline Isotope & Percentage \\
\hline $\mathrm{Pu}-238$ & $0.08 \%$ \\
\hline $\mathrm{Pu}-239$ & $94.29 \%$ \\
\hline $\mathrm{Pu}-240$ & $5.31 \%$ \\
\hline $\mathrm{Pu}-241$ & $0.24 \%$ \\
\hline $\mathrm{Pu}-242$ & $0.09 \%$ \\
\hline
\end{tabular}




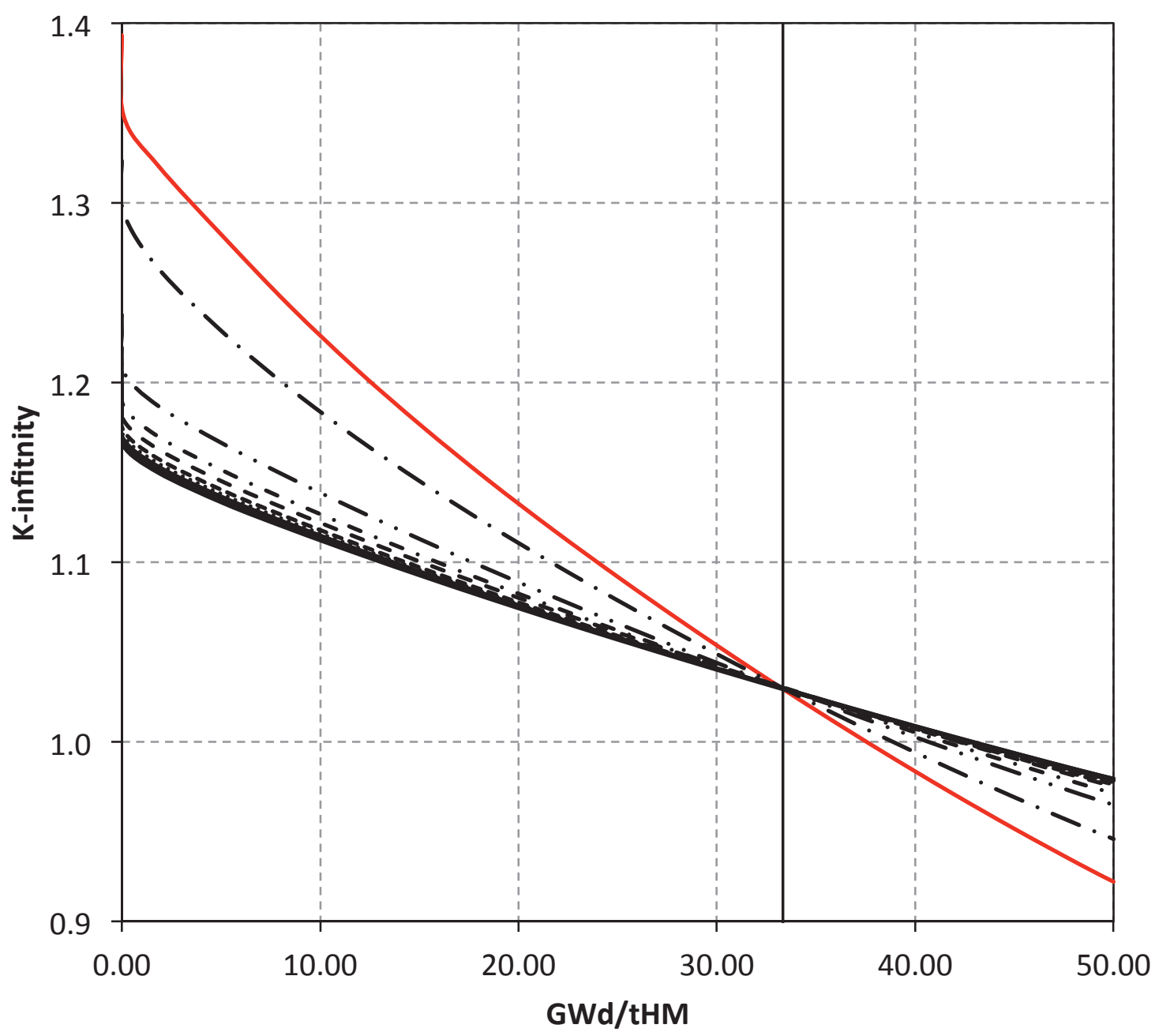

REFERENCE UOX (4.21\% U235)

- - Case E2 (Cycle 2: 5.77\% Pu, 94.23\% U-nat)

- - - Case E2 (Cycle 4: 7.37\% Pu, 92.63\% U-nat)

........ Case E2 (Cycle 6: 8.12\% Pu, 91.88\% U-nat)

- Case E2 (Cycle 8: 8.58\% Pu, 91.42\% U-nat)

— Case E2 (Cycle 10: 8.82\% Pu, 91.18\% U-nat)
—- - Case E2 (Cycle 1: 4.21\% Pu, 95.79\% U-nat)

- - Case E2 (Cycle 3: 6.71\% Pu, 93.29\% U-nat)

- - Case E2 (Cycle 5: 7.79\% Pu, 92.21\% U-nat)

Case E2 (Cycle 7: 8.38\% Pu, 91.62\% U-nat)

Case E2 (Cycle 9: 8.72\% Pu, 91.28\% U-nat)

Figure 2-3: K-infinity trends vs. burnup showing the intersection point at 33.33 GWd/tHM. 


\section{RESULTS}

\subsection{Plutonium contents}

The plutonium content considered for the first cycle is coming from the LMFBR blanket. Since the isotopic composition of the LMFBR blanket is highly fissile $(\sim 95 \%)$, the required amount of $\mathrm{Pu}$ for the first cycle is slightly over $4 \%$ by weight. The amount of $\mathrm{Pu}$ is steadily increased in the PWR MOX-NU assemblies after each cycle, since the fissile content of the $\mathrm{Pu}$ is reduced after each cycle and more $\mathrm{Pu}-240, \mathrm{Pu}-241$ and $\mathrm{Pu}-242$ is produced in each cycle. The amount of $\mathrm{Pu}$ reaches to an equilibrium roughly after $10^{\text {th }}$ cycle.

Table 3-1 and Figure 3-1 below show the calculated $P u$ content in the fuel assemblies necessary to maintain the same average burn-up at discharge of $50 \mathrm{GWd} / \mathrm{tHM}$ as a function of cycle number. Figure 3-2 shows the weight percent of each $\mathrm{Pu}$ isotope at the BOL for each cycle whereas Figure 3-3 shows the $\mathrm{Pu}$ isotopic compositions at BOL for each cycle. Figure 3-4 shows the isotopic composition $\mathrm{Pu}$ at $\mathrm{BOL}$ and EOL for each cycle. For the first cycle the destruction of $\mathrm{Pu}-239$ is about $50 \%$ of its initial value, after reaching the equilibrium the destruction of $\mathrm{Pu}-239$ per cycle reduces to about $30 \%$ of its initial value. Figure 3-5 shows the weight percentages of $\mathrm{Pu}$ at the BOL and EOL per cycle, it also shows the weight percentage of the Pu from LMFBR blanket, which is required to be mixed with the recycled $\mathrm{Pu}$ from the previous cycle to achieve the target burnup. Figure 3-6 shows the percentage of the $\mathrm{Pu}$ according to their origins (i.e. either from previous cycle or LMFBR blanket) that is loaded to the PWR MOX-NU assemblies per cycle.

Table 3-1: Pu and $U$ percentages in the fresh fuel per cycle

\begin{tabular}{|c|r|r|}
\hline Cycle & $\boldsymbol{P u}$ & $\mathbf{U}$ \\
\hline 1 & $4.21 \%$ & $95.79 \%$ \\
\hline 2 & $5.77 \%$ & $94.23 \%$ \\
\hline 3 & $6.71 \%$ & $93.29 \%$ \\
\hline 4 & $7.37 \%$ & $92.63 \%$ \\
\hline 5 & $7.79 \%$ & $92.21 \%$ \\
\hline 6 & $8.12 \%$ & $91.88 \%$ \\
\hline 7 & $8.38 \%$ & $91.62 \%$ \\
\hline 8 & $8.58 \%$ & $91.42 \%$ \\
\hline 9 & $8.72 \%$ & $91.28 \%$ \\
\hline 10 & $8.82 \%$ & $91.18 \%$ \\
\hline 11 & $8.90 \%$ & $91.10 \%$ \\
\hline 12 & $8.97 \%$ & $91.03 \%$ \\
\hline 13 & $9.03 \%$ & $90.97 \%$ \\
\hline 14 & $9.08 \%$ & $90.92 \%$ \\
\hline 15 & $9.13 \%$ & $90.87 \%$ \\
\hline
\end{tabular}




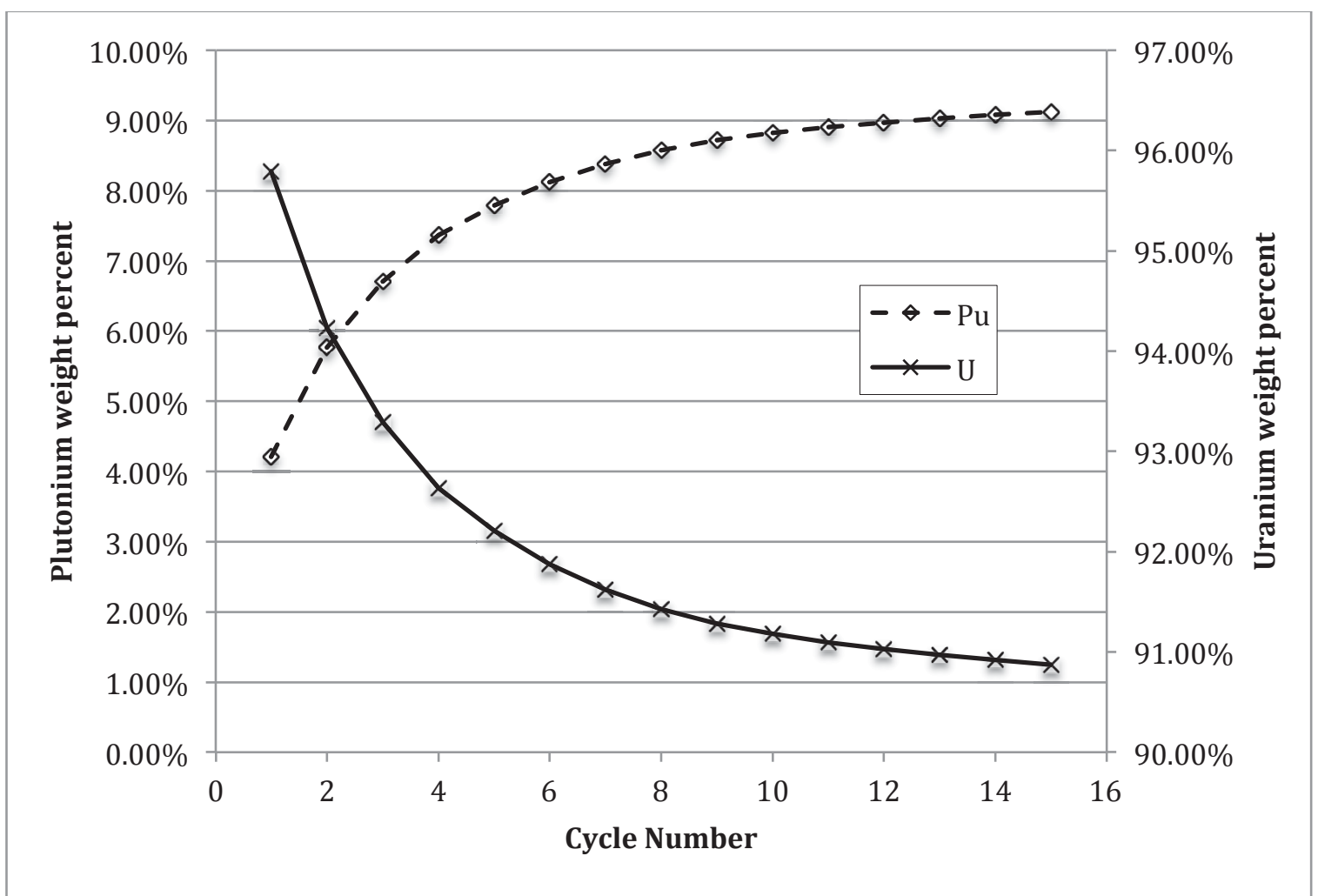

Figure 3-1: $\mathrm{U}$ and Pu percentages in PWR MOX-NU fuel assemblies per cycle.

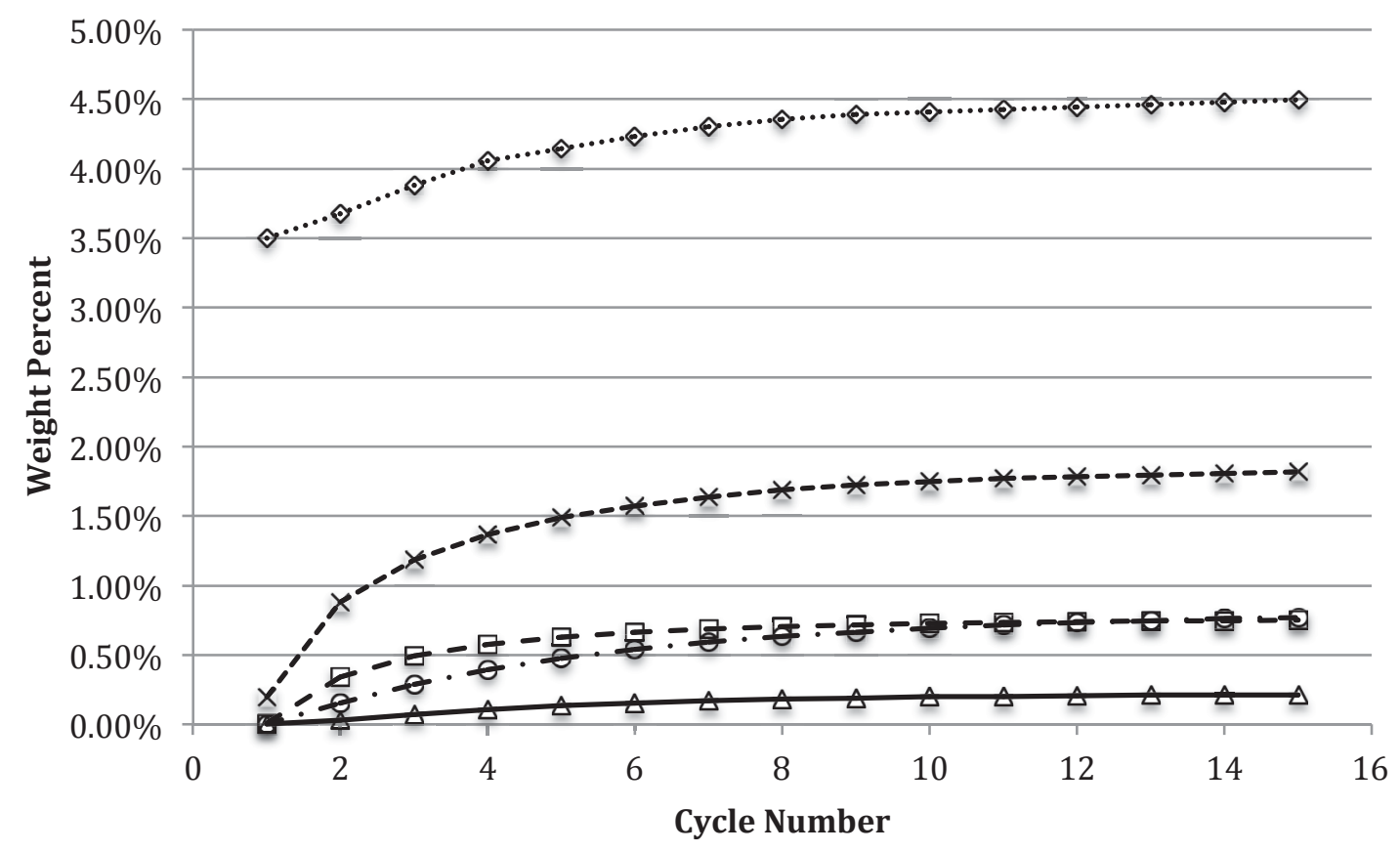

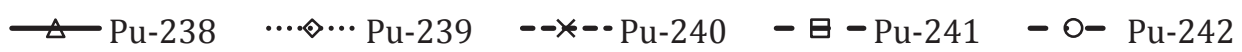

Figure 3-2: Weight percent of Pu isotopes in PWR MOX-NU assemblies per cycle 
Multirecycling of Plutonium from LMFBR Blanket in Standard PWRS Loaded with MOX Fuel

February 2013

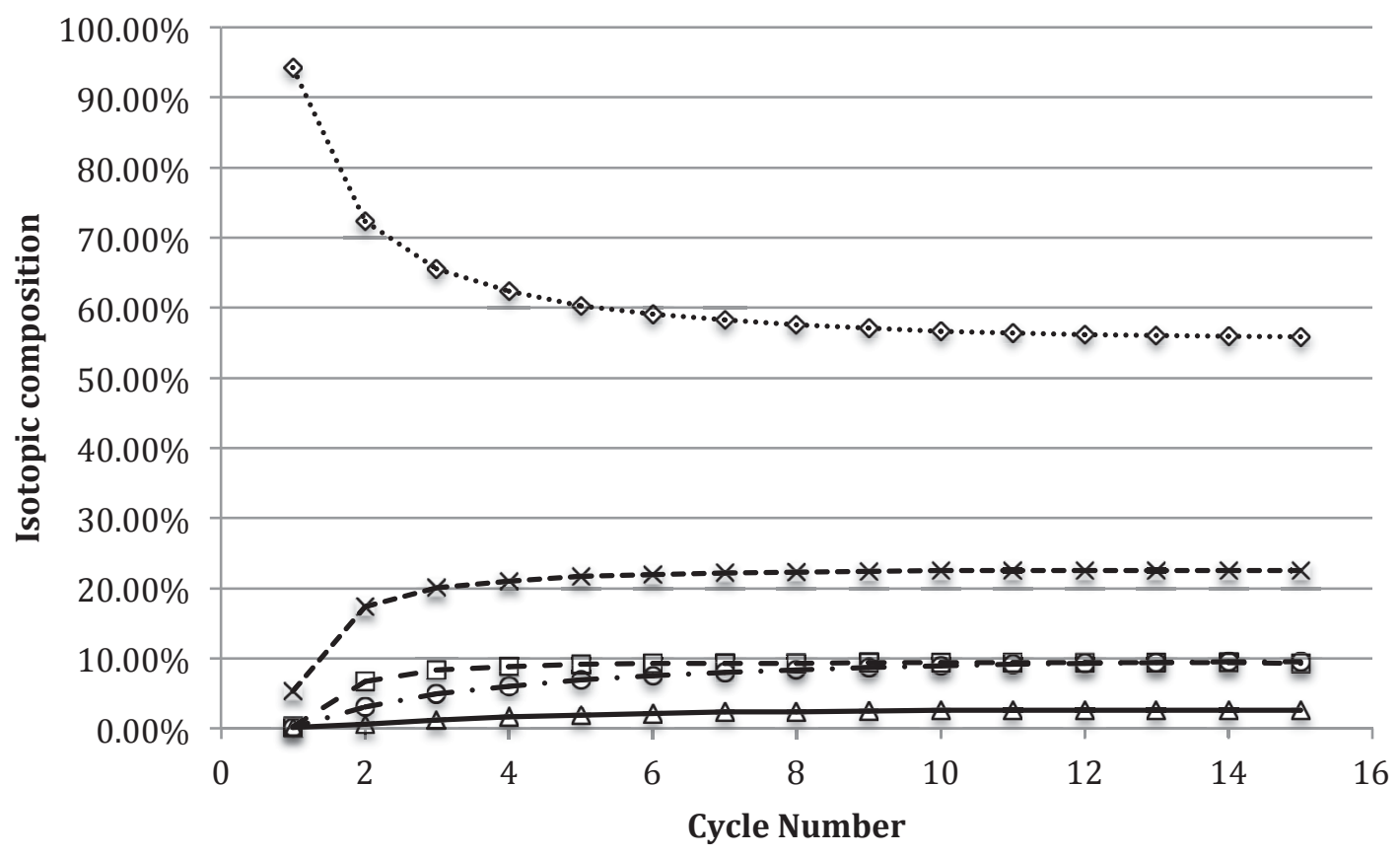

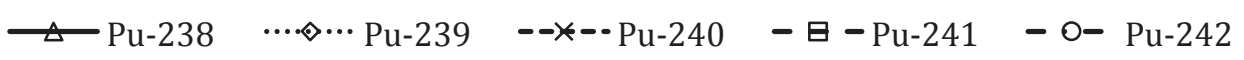

Figure 3-3: Isotopic composition of Pu in PWR MOX-NU assemblies per cycle (BOL only) 


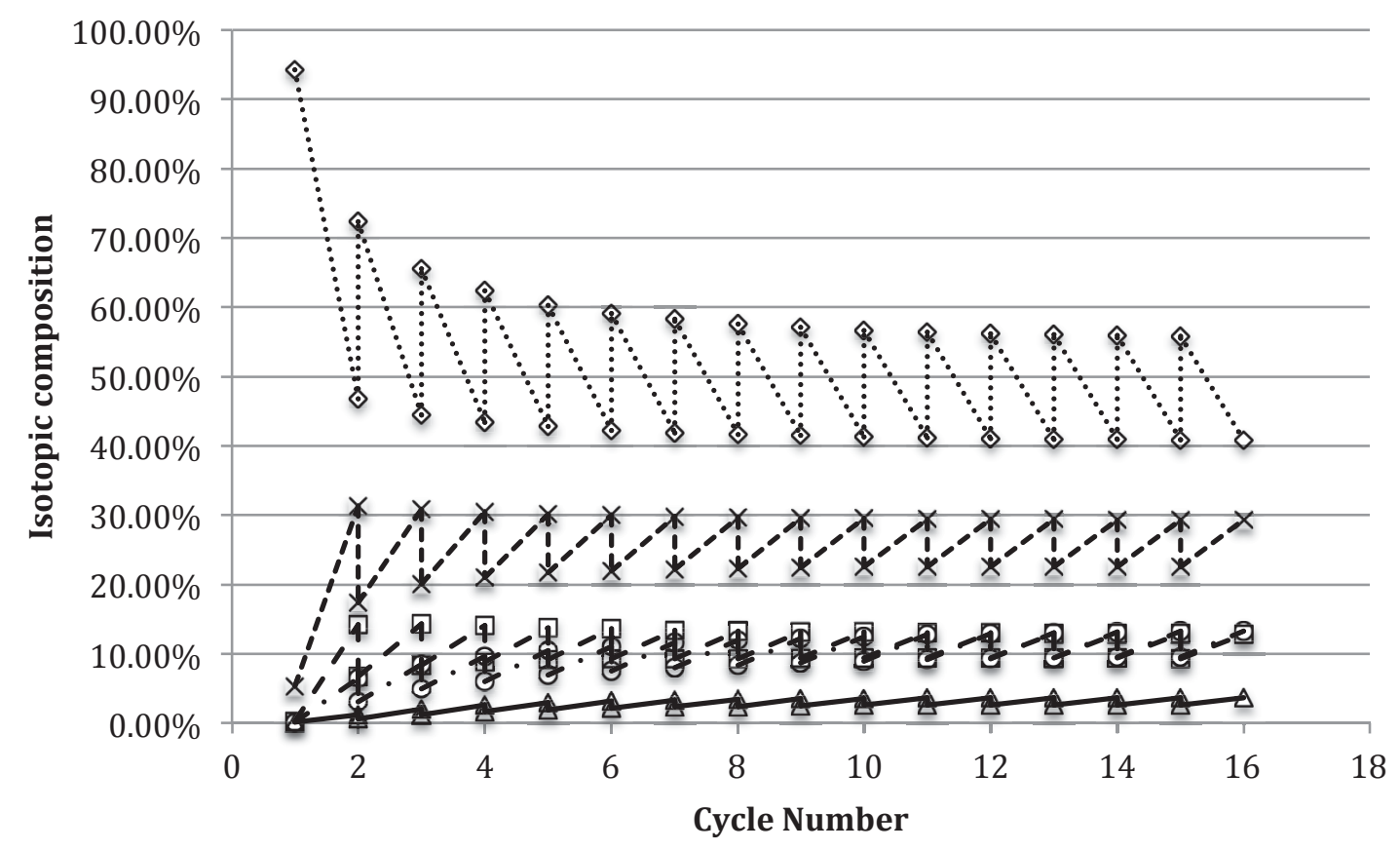

$\triangle \mathrm{Pu}-238 \quad \cdots \otimes \cdots \mathrm{Pu}-239 \quad--\rtimes--\mathrm{Pu}-240 \quad-\quad$ - $-\mathrm{Pu}-241 \quad-\mathrm{O}-\mathrm{Pu}-242$

Figure 3-4: Isotopic composition of Pu in PWR MOX-NU assemblies per cycle (BOL and EOL)

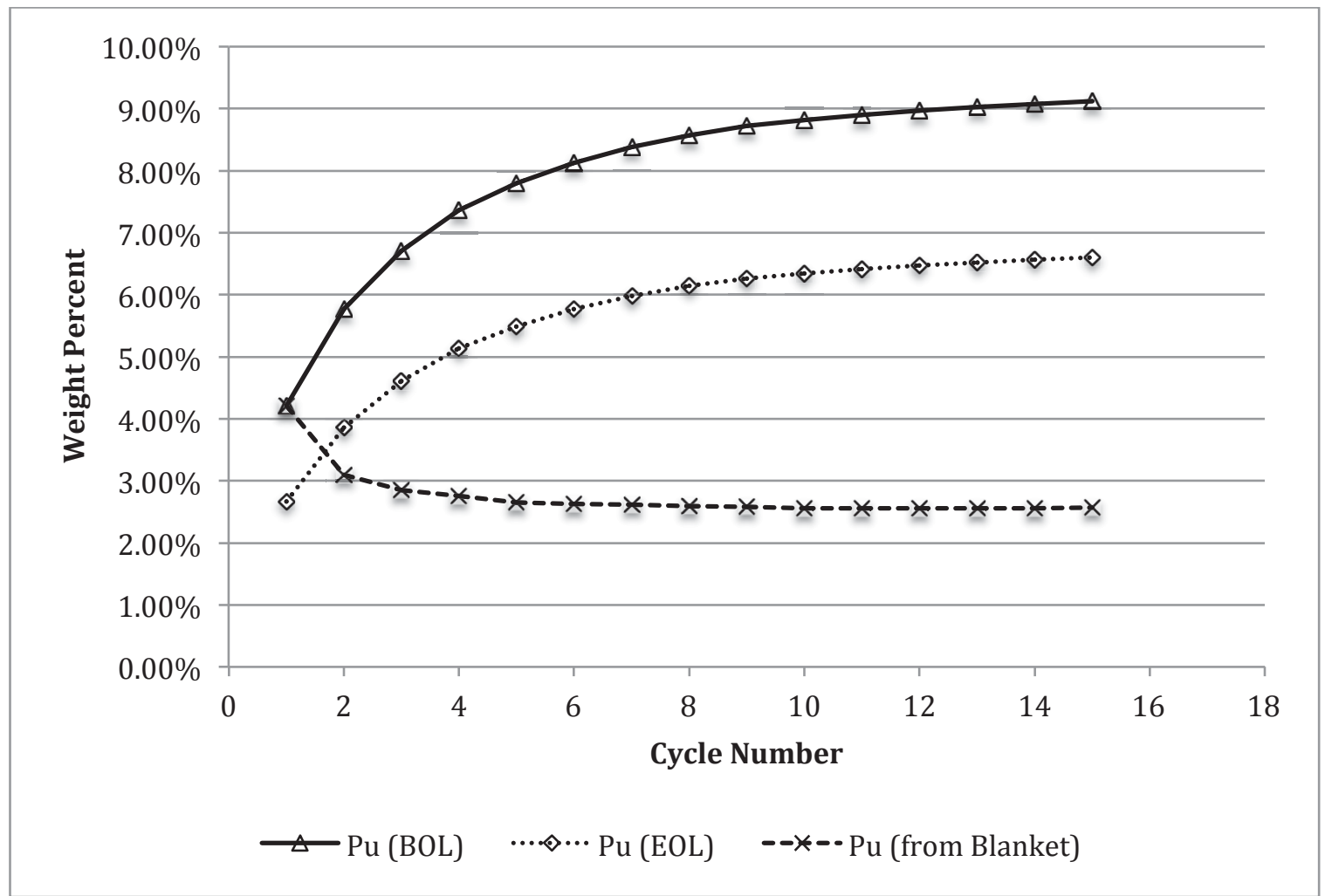

Figure 3-5: Weight percentages of Pu in PWR MOX-NU assemblies per cycle 
Multirecycling of Plutonium from LMFBR Blanket in Standard PWRS Loaded with MOX Fuel

February 2013

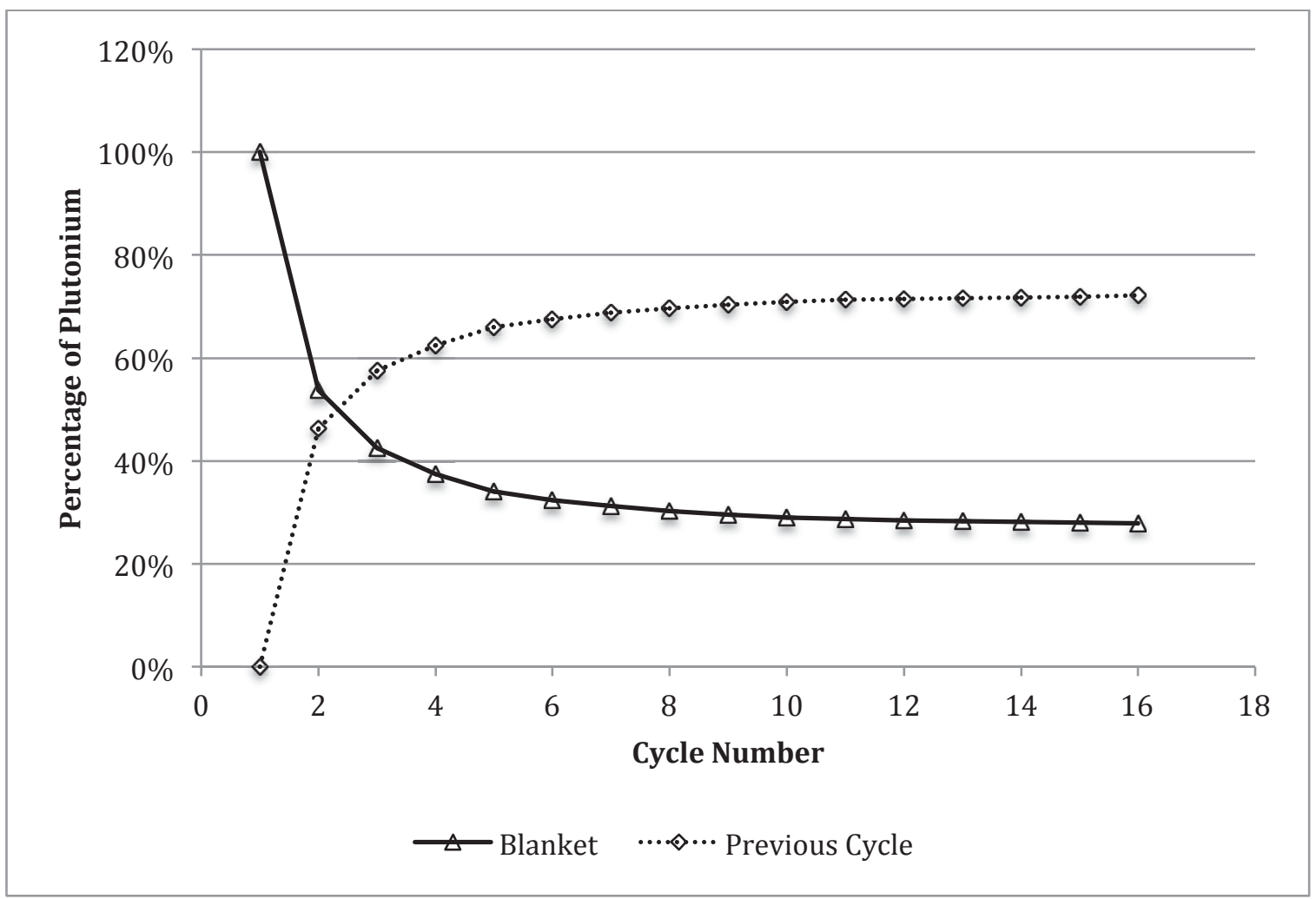

Figure 3-6: The origin of Pu in PWR MOX-NU assemblies per cycle 
Figure 3-7 shows the infinite multiplication factor trends for each cycle step (initial fuel aging, burning) against the $50 \mathrm{GWd} / \mathrm{tHM} \mathrm{UOX}(4.2 \%)$ reference case.

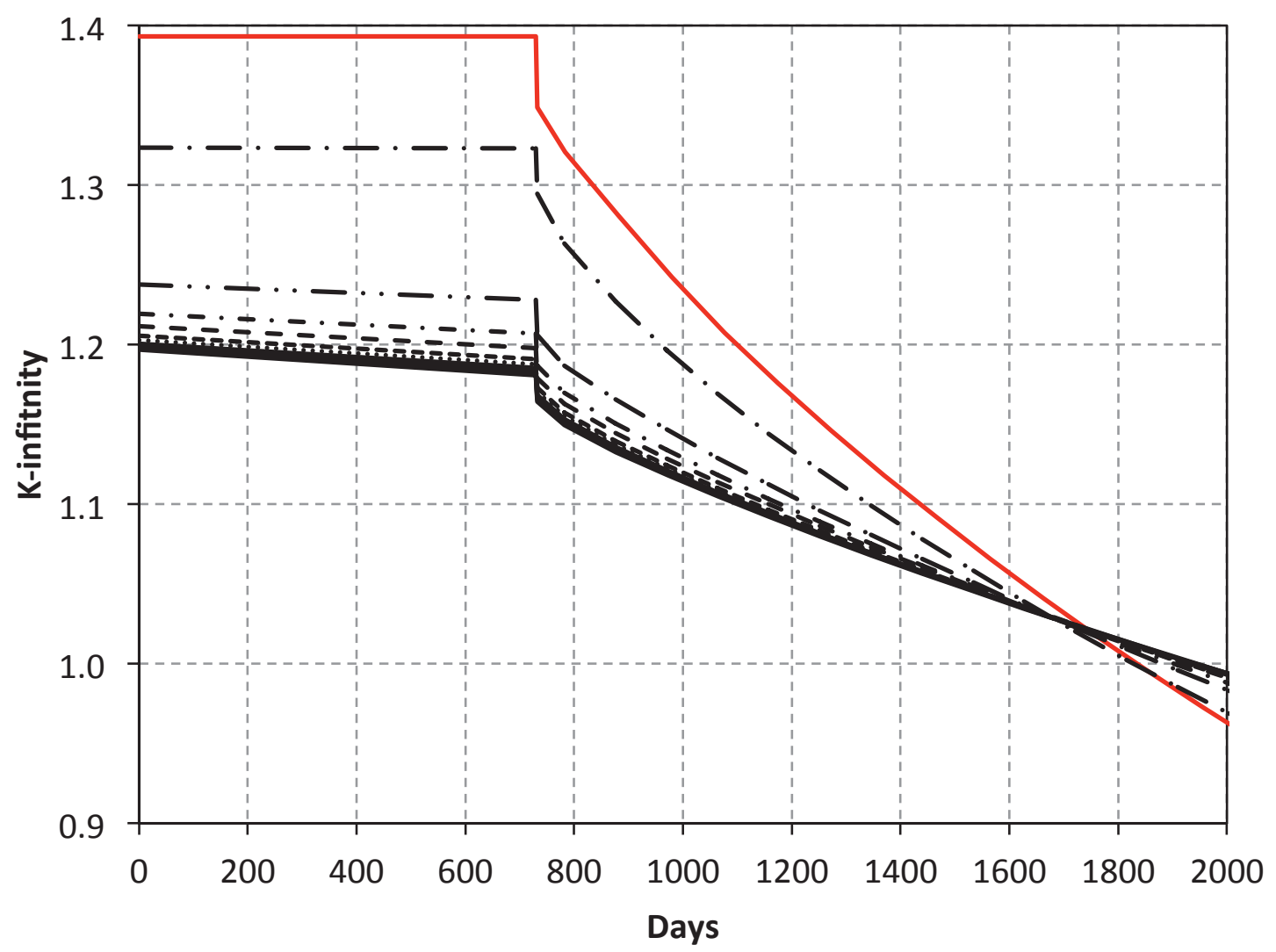

- REFERENCE UOX (4.21 \% U235)
— - C Case E2 (Cycle 2: 5.77\% Pu, 94.23\% U-nat)
— - Case E2 (Cycle 4: 7.37\% Pu, 92.63\% U-nat)
…... Case E2 (Cycle 6: 8.12\% Pu, 91.88\% U-nat)
— Case E2 (Cycle 8: 8.58\% Pu, 91.42\% U-nat)
Case E2 (Cycle 10: 8.82\% Pu, 91.18\% U-nat)

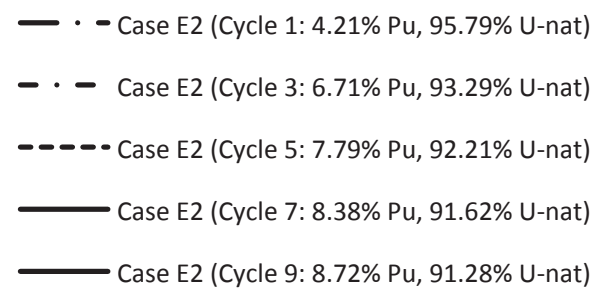

Figure 3-7: $k$-infinitive trends for the first 10 cycles.

Table 3-2 summarizes the isotopic input, after 2 years aging, and output compositions, at the average discharged burn-up of $50 \mathrm{GWd} / \mathrm{tHM}$, for the four cases taken in exam. 


\section{Multirecycling of Plutonium from LMFBR Blanket in Standard PWRS Loaded with}

MOX Fuel

February 2013

Table 3-2: Mass Fraction input and output (summary).

\begin{tabular}{|c|c|c|c|c|c|c|c|c|}
\hline \multicolumn{9}{|c|}{ Isotopic input ( 2 yrs aging) and output mass vectors } \\
\hline \multirow{2}{*}{ Isotope } & \multicolumn{2}{|c|}{$1^{\text {st }}$ Cycle } & \multicolumn{2}{|c|}{$2^{\text {nd }}$ Cycle } & \multicolumn{2}{|c|}{$5^{\text {th }}$ Cycle } & \multicolumn{2}{|c|}{$10^{\text {th }}$ Cycle } \\
\hline & \multicolumn{2}{|c|}{$\mathrm{kg} / \mathrm{tIH} M$} & \multicolumn{2}{|c|}{$\mathrm{kg} / \mathrm{tIH}$} & \multicolumn{2}{|c|}{$\mathrm{kg} / \mathrm{tIH}$} & \multicolumn{2}{|c|}{$\mathrm{kg} / \mathrm{tIHM}$} \\
\hline IDs & Input & Output & Input & Output & Input & Output & nput & Output \\
\hline U232 & $0.000 \mathrm{E}+00$ & $0.000 \mathrm{E}+00$ & $.000 \mathrm{E}+00$ & $0.000 \mathrm{E}+00$ & $0.000 \mathrm{E}+00$ & $0.000 \mathrm{E}+00$ & $0.000 \mathrm{E}+00$ & $0.000 \mathrm{E}+00$ \\
\hline U233 & $5.196 \mathrm{E}-09$ & $1.210 \mathrm{E}-03$ & $1.264 \mathrm{E}-07$ & $1.585 \mathrm{E}-03$ & $2.327 \mathrm{E}-07$ & $2.472 \mathrm{E}-03$ & $2.692 \mathrm{E}-07$ & $3.003 \mathrm{E}-03$ \\
\hline U234 & $5.221 \mathrm{E}+01$ & $3.011 \mathrm{E}+01$ & $5.594 \mathrm{E}+01$ & $4.152 \mathrm{E}+01$ & $7.316 \mathrm{E}+01$ & $7.660 \mathrm{E}+01$ & $8.382 \mathrm{E}+01$ & $9.801 \mathrm{E}+01$ \\
\hline$U 235$ & $6.812 \mathrm{E}+03$ & $2.385 \mathrm{E}+03$ & $6.701 \mathrm{E}+03$ & $2.850 \mathrm{E}+03$ & $6.557 \mathrm{E}+03$ & $3.216 \mathrm{E}+03$ & $6.484 \mathrm{E}+03$ & $3.335 \mathrm{E}+03$ \\
\hline$U 236$ & 646E-01 & $8.478 \mathrm{E}+02$ & $2.078 \mathrm{E}+00$ & $7.933 \mathrm{E}+02$ & $3.510 \mathrm{E}+00$ & $7.386 \mathrm{E}+02$ & $4.123 \mathrm{E}+00$ & $7.161 \mathrm{E}+02$ \\
\hline U238 & $9.508 \mathrm{E}+05$ & $9.167 \mathrm{E}+05$ & $9.354 \mathrm{E}+05$ & $9.026 \mathrm{E}+05$ & $9.153 \mathrm{E}+05$ & $8.839 \mathrm{E}+05$ & $9.051 \mathrm{E}+05$ & $8.743 \mathrm{E}+05$ \\
\hline$N p 237$ & $528 \mathrm{E}-02$ & $2.410 \mathrm{E}+02$ & $5.776 \mathrm{E}-01$ & $2.429 \mathrm{E}+02$ & $1.069 \mathrm{E}+00$ & $2.418 \mathrm{E}+02$ & $1.238 \mathrm{E}+00$ & $2.407 \mathrm{E}+02$ \\
\hline Pu238 & $3.148 \mathrm{E}+01$ & $2.388 \mathrm{E}+02$ & $3.200 \mathrm{E}+02$ & $6.670 \mathrm{E}+02$ & $1.493 \mathrm{E}+03$ & $1.620 \mathrm{E}+03$ & $2.210 \mathrm{E}+03$ & $2.172 \mathrm{E}+03$ \\
\hline Pu239 & $3.968 \mathrm{E}+04$ & $1.241 \mathrm{E}+04$ & $4.169 \mathrm{E}+04$ & $1.706 \mathrm{E}+04$ & $4.699 \mathrm{E}+04$ & $2.310 \mathrm{E}+04$ & $4.999 \mathrm{E}+04$ & $2.604 \mathrm{E}+04$ \\
\hline Pu240 & $2.233 \mathrm{E}+03$ & $8.333 \mathrm{E}+03$ & $1.000 \mathrm{E}+04$ & $1.181 \mathrm{E}+04$ & $1.690 \mathrm{E}+04$ & $1.629 \mathrm{E}+04$ & $1.985 \mathrm{E}+04$ & $1.852 \mathrm{E}+04$ \\
\hline Pu241 & $9.255 \mathrm{E}+01$ & $4.816 \mathrm{E}+03$ & $3.498 \mathrm{E}+03$ & $7.035 \mathrm{E}+03$ & $6.475 \mathrm{E}+03$ & $9.499 \mathrm{E}+03$ & $7.495 \mathrm{E}+03$ & $1.055 \mathrm{E}+04$ \\
\hline Pu242 & $3.652 \mathrm{E}+01$ & $1.738 \mathrm{E}+03$ & $1.765 \mathrm{E}+03$ & $3.254 \mathrm{E}+03$ & $5.369 \mathrm{E}+03$ & $6.084 \mathrm{E}+03$ & $7.849 \mathrm{E}+03$ & $8.085 \mathrm{E}+03$ \\
\hline$A m 241$ & $9.405 \mathrm{E}+00$ & $2.689 \mathrm{E}+02$ & $3.555 \mathrm{E}+02$ & $5.740 \mathrm{E}+02$ & $6.581 \mathrm{E}+02$ & $9.670 \mathrm{E}+02$ & $7.618 \mathrm{E}+02$ & $1.146 \mathrm{E}+03$ \\
\hline$A m 242 m$ & 14E-06 & $4.628 \mathrm{E}+00$ & $4.457 \mathrm{E}-05$ & $1.151 \mathrm{E}+01$ & $7.145 \mathrm{E}-05$ & $2.169 \mathrm{E}+01$ & $7.800 \mathrm{E}-05$ & $2.666 \mathrm{E}+01$ \\
\hline$A m 243$ & $1.186 \mathrm{E}-06$ & $4.558 \mathrm{E}+02$ & $2.969 \mathrm{E}-06$ & $1.053 \mathrm{E}+03$ & $4.786 \mathrm{E}-06$ & $1.755 \mathrm{E}+03$ & $5.541 \mathrm{E}-06$ & $2.097 \mathrm{E}+03$ \\
\hline $\mathrm{Cm} 242$ & $5.848 \mathrm{E}-08$ & $7.484 \mathrm{E}+01$ & $2.209 \mathrm{E}-07$ & $1.379 \mathrm{E}+02$ & $3.225 \mathrm{E}-07$ & $1.876 \mathrm{E}+02$ & $3.467 \mathrm{E}-07$ & $2.031 \mathrm{E}+02$ \\
\hline$C m 243$ & 31E-06 & $2.290 \mathrm{E}+00$ & $1.081 \mathrm{E}-06$ & $5.762 \mathrm{E}+00$ & $1.081 \mathrm{E}-06$ & $8.452 \mathrm{E}+00$ & $1.081 \mathrm{E}-06$ & $9.248 \mathrm{E}+00$ \\
\hline $\mathrm{Cm} 244$ & $1.051 \mathrm{E}-06$ & $1.981 \mathrm{E}+02$ & $1.051 \mathrm{E}-06$ & $6.460 \mathrm{E}+02$ & $1.051 \mathrm{E}-06$ & $1.032 \mathrm{E}+03$ & $1.051 \mathrm{E}-06$ & $1.182 \mathrm{E}+03$ \\
\hline$C m 245$ & $1.134 \mathrm{E}-06$ & $1.880 \mathrm{E}+01$ & $1.134 \mathrm{E}-06$ & $8.193 \mathrm{E}+01$ & $1.134 \mathrm{E}-06$ & $1.347 \mathrm{E}+02$ & 1.134E-06 & $1.535 \mathrm{E}+02$ \\
\hline $\mathrm{Cm} 246$ & $1.134 \mathrm{E}-06$ & $1.623 \mathrm{E}+00$ & $1.134 \mathrm{E}-06$ & $7.381 \mathrm{E}+00$ & $1.134 \mathrm{E}-06$ & $9.519 \mathrm{E}+00$ & $1.134 \mathrm{E}-06$ & $9.834 \mathrm{E}+00$ \\
\hline Cm247 & E-06 & $2.720 \mathrm{E}-02$ & E-06 & 39E-01 & -06 & $1.983 \mathrm{E}-01$ & E-06 & E-01 \\
\hline $\mathrm{Cm} 248$ & $1.134 \mathrm{E}-06$ & $2.018 \mathrm{E}-03$ & $1.134 \mathrm{E}-06$ & $1.248 \mathrm{E}-02$ & $1.134 \mathrm{E}-06$ & $1.428 \mathrm{E}-02$ & $1.134 \mathrm{E}-06$ & $1.393 \mathrm{E}-02$ \\
\hline$B k 249$ & $0.000 \mathrm{E}+00$ & $0.000 \mathrm{E}+00$ & $0.000 \mathrm{E}+00$ & $0.000 \mathrm{E}+00$ & $0.000 \mathrm{E}+00$ & $0.000 \mathrm{E}+00$ & $0.000 \mathrm{E}+00$ & $0.000 \mathrm{E}+00$ \\
\hline$C f 249$ & $0.000 \mathrm{E}+00$ & $0.000 \mathrm{E}+00$ & $0.000 \mathrm{E}+00$ & $0.000 \mathrm{E}+00$ & $0.000 \mathrm{E}+00$ & $0.000 \mathrm{E}+00$ & $0.000 \mathrm{E}+00$ & $0.000 \mathrm{E}+00$ \\
\hline & & & (3) & tad & It a s mp & & & \\
\hline IDs & $\%$ & $\%$ & $\%$ & $\%$ & $\%$ & 10 & $\%$ & $\%$ \\
\hline$I$ & $95.77 \%$ & $92.00 \%$ & $94.22 \%$ & $90.63 \%$ & $92.19 \%$ & $88.79 \%$ & $91.17 \%$ & $87.84 \%$ \\
\hline$N p$ & $0.00 \%$ & $0.02 \%$ & $0.00 \%$ & $0.02 \%$ & $0.00 \%$ & $0.02 \%$ & $0.00 \%$ & $0.02 \%$ \\
\hline$P u$ & $4.21 \%$ & $2.75 \%$ & $5.73 \%$ & $3.98 \%$ & $7.72 \%$ & $5.66 \%$ & $8.74 \%$ & $6.54 \%$ \\
\hline$A m$ & $0.00 \%$ & $0.07 \%$ & $0.04 \%$ & $0.16 \%$ & $0.07 \%$ & $0.27 \%$ & $0.08 \%$ & $0.33 \%$ \\
\hline
\end{tabular}




\begin{tabular}{|c|c|c|c|c|c|c|c|c|}
\hline$C m$ & $0.00 \%$ & $0.03 \%$ & $0.00 \%$ & $0.09 \%$ & $0.00 \%$ & $0.14 \%$ & $0.00 \%$ & $0.16 \%$ \\
\hline$B k$ & $0.00 \%$ & $0.00 \%$ & $0.00 \%$ & $0.00 \%$ & $0.00 \%$ & $0.00 \%$ & $0.00 \%$ & $0.00 \%$ \\
\hline$C f$ & $0.00 \%$ & $0.00 \%$ & $0.00 \%$ & $0.00 \%$ & $0.00 \%$ & $0.00 \%$ & $0.00 \%$ & $0.00 \%$ \\
\hline$F P$ & $0.00 \%$ & $5.11 \%$ & $0.00 \%$ & $5.11 \%$ & $0.00 \%$ & $5.10 \%$ & $0.00 \%$ & $5.10 \%$ \\
\hline
\end{tabular}

\subsection{Reactivity Coefficients}

The reactivity coefficients are very important for reactor safety and should be negative to ensure negative feedback. This section presents the results of a reactivity coefficient analysis of the MOX-NU fueled PWR. The fuel and moderator temperature, and void coefficients are looked at. For MOX fueled PWRs the void coefficient tends to become less negative when the total plutonium content is increased. In the conventional PWR lattice changes sign from negative to positiove at a total plutonium content of between $10 \%$ and $12 \%$ depending on its isotopic composition [6]. Since the Pu content at the equilibrium has not been reached to these levels, only the results is shown here and no attempt to make the void coefficient more negative has been done. For a more detailed analysis on the void coefficient issue refer [1]. Only the reactivity coefficients for the $15^{\text {th }}$ cycle will be presented here since the Pu content is the highest for this cycle. Table 3-3 shows the reactivity coefficients at the BOL (after 2 years of aging).

Table 3-3: Reactivity Coefficients calculated for the $15^{\text {th }}$ cycle.

\begin{tabular}{|l|r|}
\hline Coefficient & Value \\
\hline Fuel Temperature $\left(\mathrm{pcm} /{ }^{\circ} \mathrm{C}\right)$ & -2.669 \\
\hline Moderator Temperature $\left(\mathrm{pcm} /{ }^{\circ} \mathrm{C}\right)$ & -41.069 \\
\hline Void $(90 \%)(\mathrm{pcm})$ & -13143.513 \\
\hline
\end{tabular}




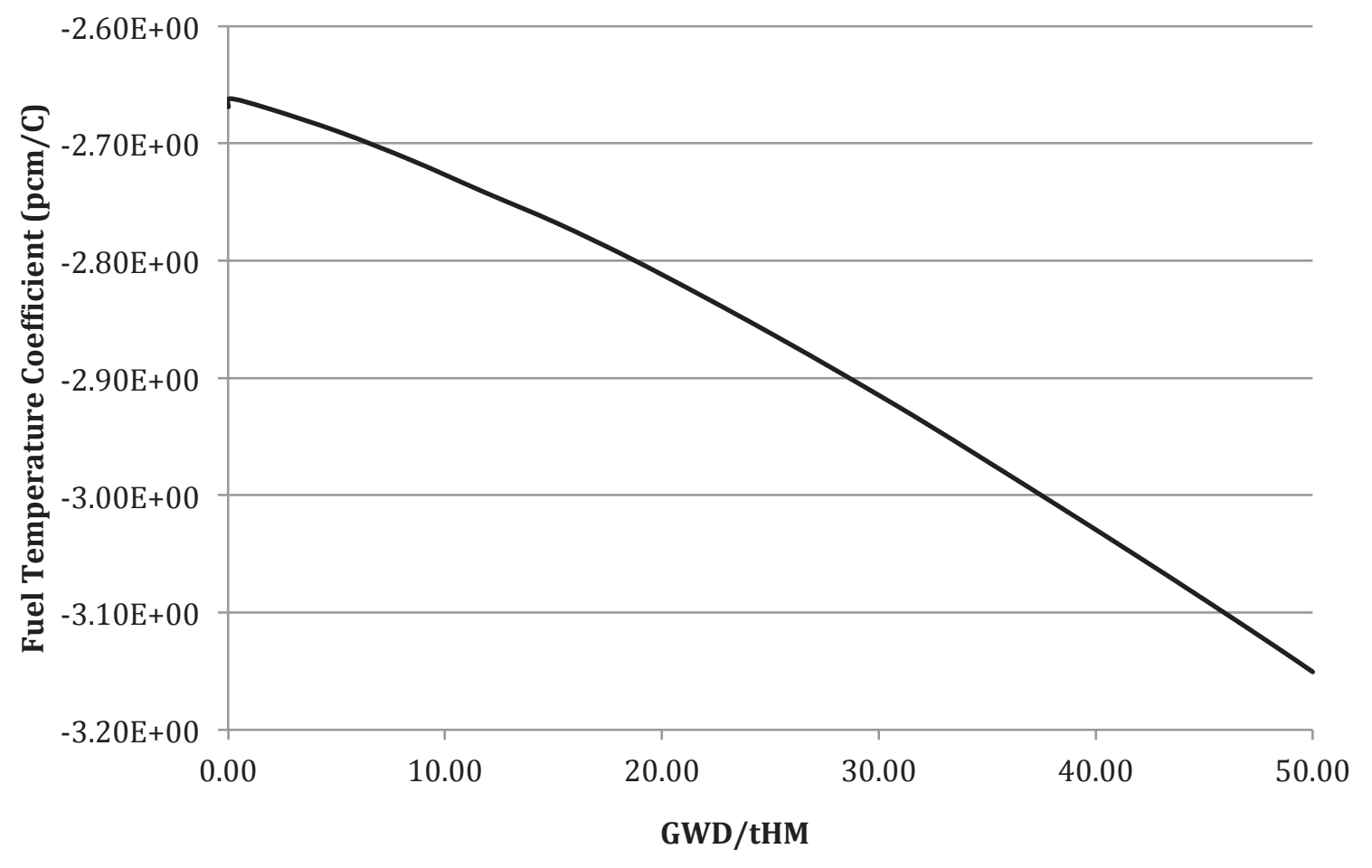

Figure 3-8: Fuel Temperature coefficient for the 15th Cycle

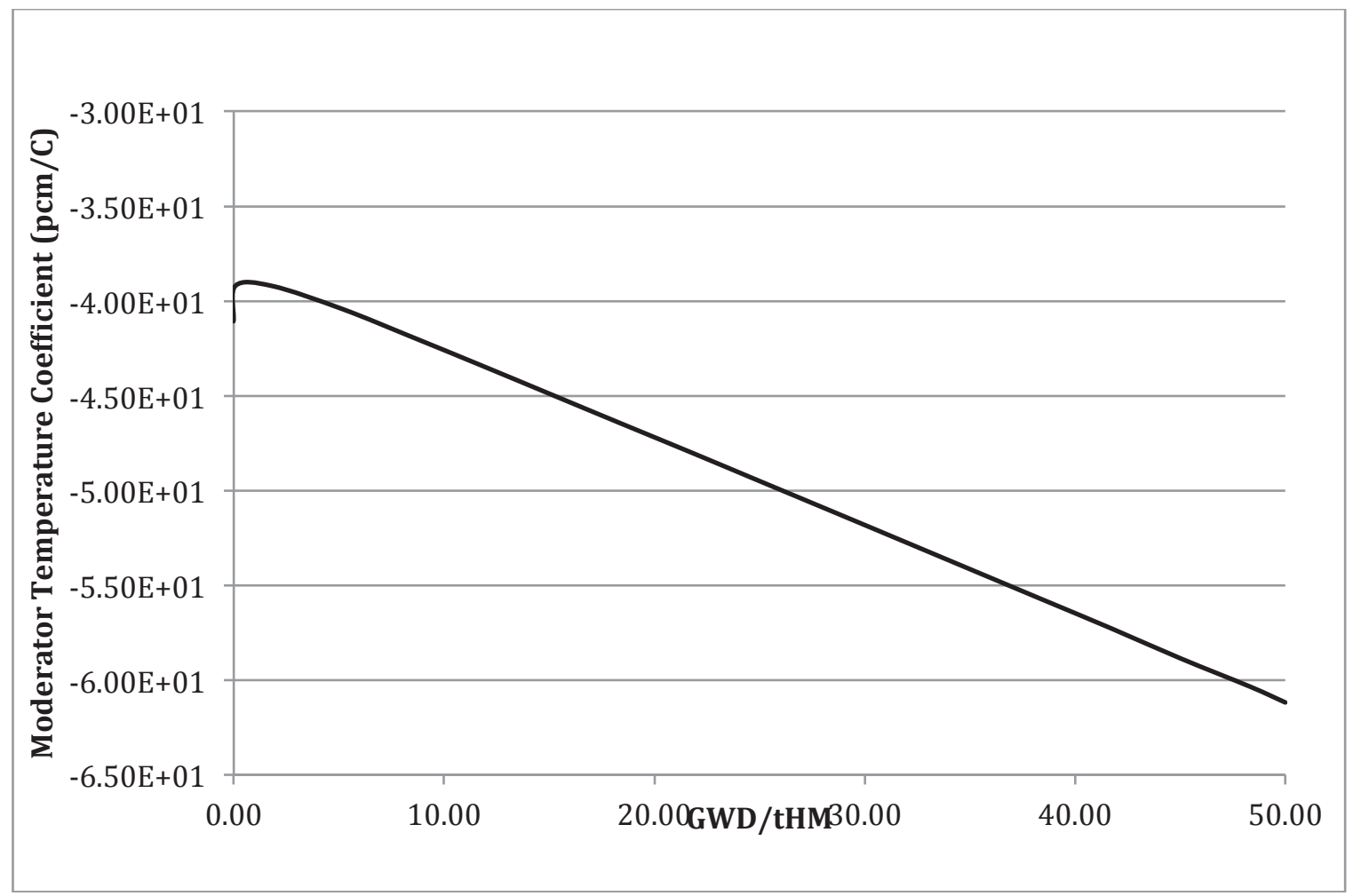

Figure 3-9: Moderator Temperature coefficient for the 15th Cycle 


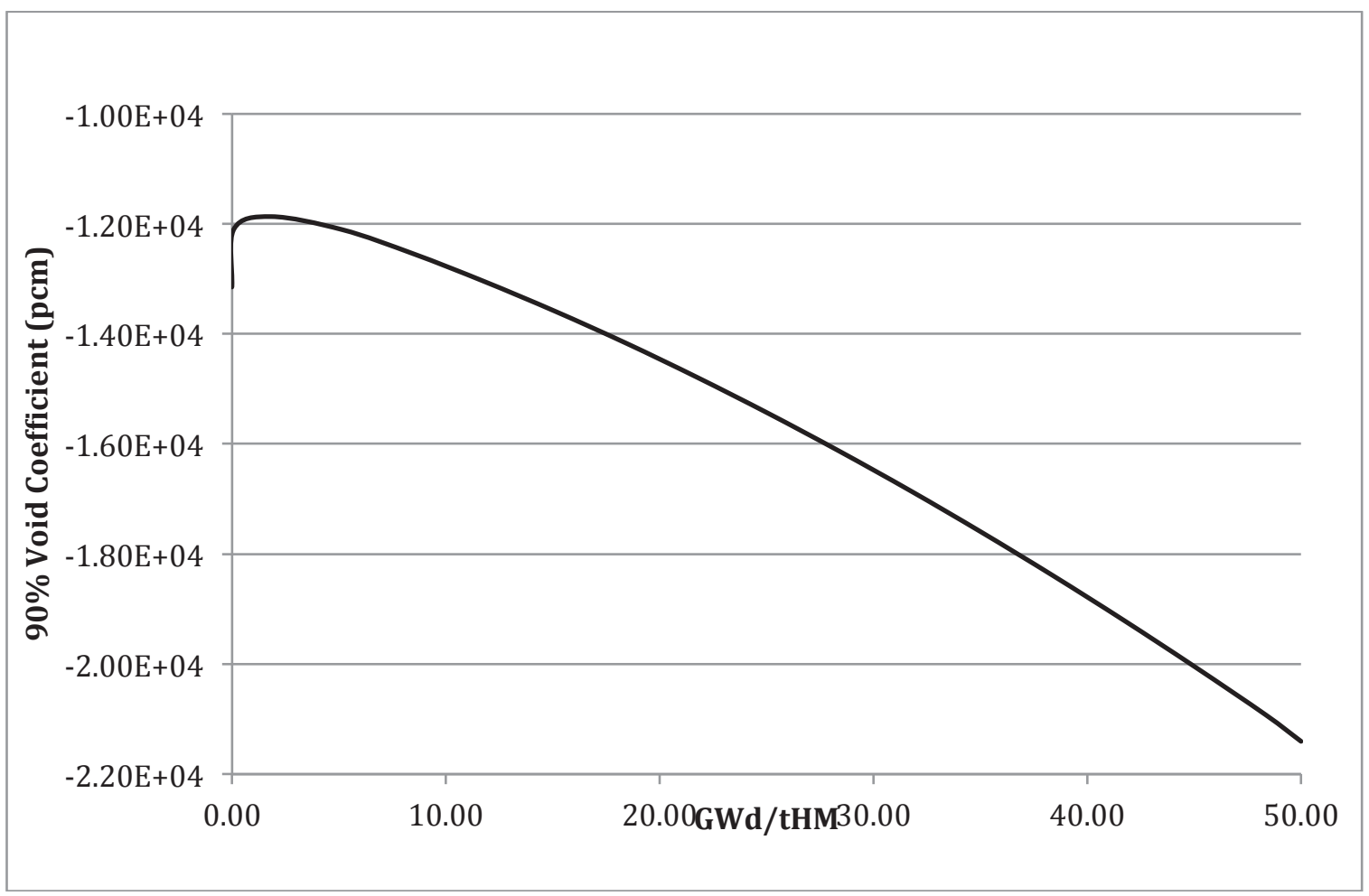

Figure 3-10: 90\% Void coefficient for the 15th cycle 


\section{CONCLUSIONS.}

This report presents the results of a neutronics analysis in which the option of a MOX-NU based fuel cycle has been shown.

The main objective of these calculations is to quantify the Pu content needed to operate a MOXNU based reactor (PWR) in order to maintain the same average burnup at discharge (50 $\mathrm{GWd} / \mathrm{tHM}$ ) and, consequently, collect the data needed for further analysis.

It is important to recognize that these results are based on infinite lattice assembly calculations, assuming standard UOX fuel assembly parameters with homogeneously loaded fuel. While this approach increases the ability to retrofit MOX based fuels into existing commercial PWRs with minimal or no changes required to reactor hardware, it does not represent the optimum performance achievable. Additionally more detailed studies (i.e. optimization of assembly and reactor parameters, calculation of void coefficients, etc.) would be required. 


\section{REFERENCES.}

1. G. Youinou, S. Bays, "A Neutronic Analysis of TRU Recycling in PWRs Loaded with MOXUE Fuel (MOX with U-235 Enriched U Support)", INL/EXT-09-16091

2. T. A. Taiwo, T. K. Kim, M. Salvatores, "Feasibility Study of a Proliferation Resistant Fuel Cycle for LWR-Based Transmutation of Transuranics", ANL-AAA-027

3. L.Gauld, O. Herman, et al., "Origen-S: Scale system module to calculate fuel depletion, Actinide transmutation, fission product buildup and decay, and associated radiation source terms", Oak Ridge National Laboratory (2009).

4. M. DeHart, "Lattice Physics Capabilities of the SCALE Code System Using TRITON", Physor Conference (2006).

5. S. Bays, "Analysis of Pu-Only Partitioning Strategies in LMFBR Fuel Cycles", INL/EXT-1328492

6. M. Salvatores, G. Youinou, R. N. Hill, T. Taiwo, T. K .Kim. "Systematic Assessment of LWR Recycle Strategies”, Document ANL-AFCI-100 (2003). 


\section{Appendix 1: Detailed isotopic mass fraction input (after 2-yrs fuel aging).}

\begin{tabular}{|c|c|c|c|c|c|}
\hline \multicolumn{6}{|c|}{ Isotopic input mass vector } \\
\hline Isotope & $1^{\text {st }}$ Cycle & $2^{\text {nd }}$ Cycle & $5^{\text {th }}$ Cycle & $10^{\text {th }}$ Cycle & $15^{\text {th }}$ Cycle \\
\hline$I D s$ & $\mathrm{~kg} / \mathrm{tIH}$ & $\mathrm{kg} / \mathrm{tIH} M$ & $\mathrm{~kg} / \mathrm{tIHM}$ & $\mathrm{kg} / \mathrm{tIHM}$ & $\mathrm{kg} / \mathrm{tIH}$ \\
\hline $\mathrm{He} 4$ & $5.47 \mathrm{E}-02$ & $1.71 \mathrm{E}-01$ & $5.23 \mathrm{E}-01$ & $7.31 \mathrm{E}-01$ & $7.81 \mathrm{E}-01$ \\
\hline$R a 228$ & $4.11 \mathrm{E}-19$ & $1.84 \mathrm{E}-18$ & $3.11 \mathrm{E}-18$ & $3.65 \mathrm{E}-18$ & $3.79 \mathrm{E}-18$ \\
\hline Th228 & $1.13 \mathrm{E}-16$ & $1.28 \mathrm{E}-16$ & $1.54 \mathrm{E}-16$ & $1.69 \mathrm{E}-16$ & $1.72 \mathrm{E}-16$ \\
\hline Th229 & $5.40 \mathrm{E}-14$ & $3.19 \mathrm{E}-13$ & $5.56 \mathrm{E}-13$ & $6.39 \mathrm{E}-13$ & $6.58 \mathrm{E}-13$ \\
\hline Th230 & $2.88 \mathrm{E}-04$ & $2.96 \mathrm{E}-04$ & $3.41 \mathrm{E}-04$ & $3.69 \mathrm{E}-04$ & $3.76 \mathrm{E}-04$ \\
\hline Th231 & $2.78 \mathrm{E}-08$ & $2.74 \mathrm{E}-08$ & $2.68 \mathrm{E}-08$ & $2.65 \mathrm{E}-08$ & $2.65 \mathrm{E}-08$ \\
\hline Th232 & $1.40 \mathrm{E}-08$ & $6.08 \mathrm{E}-08$ & $1.02 \mathrm{E}-07$ & $1.20 \mathrm{E}-07$ & $1.25 \mathrm{E}-07$ \\
\hline$P a 231$ & $1.33 \mathrm{E}-05$ & $1.30 \mathrm{E}-05$ & $1.28 \mathrm{E}-05$ & $1.26 \mathrm{E}-05$ & $1.26 \mathrm{E}-05$ \\
\hline$P a 233$ & $5.18 \mathrm{E}-10$ & $1.96 \mathrm{E}-08$ & $3.62 \mathrm{E}-08$ & 4.19E-08 & $4.32 \mathrm{E}-08$ \\
\hline$P a 234$ & $1.60 \mathrm{E}-07$ & $1.57 \mathrm{E}-07$ & $1.54 \mathrm{E}-07$ & $1.52 \mathrm{E}-07$ & $1.52 \mathrm{E}-07$ \\
\hline U232 & $8.55 \mathrm{E}-13$ & $9.84 \mathrm{E}-13$ & $1.30 \mathrm{E}-12$ & $1.48 \mathrm{E}-12$ & $1.53 \mathrm{E}-12$ \\
\hline$U 233$ & $5.20 \mathrm{E}-09$ & $1.26 \mathrm{E}-07$ & $2.33 \mathrm{E}-07$ & $2.69 \mathrm{E}-07$ & $2.78 \mathrm{E}-07$ \\
\hline$U 234$ & $5.22 \mathrm{E}+01$ & $5.59 \mathrm{E}+01$ & $7.32 \mathrm{E}+01$ & $8.38 \mathrm{E}+01$ & $8.64 \mathrm{E}+01$ \\
\hline$U 235$ & $6.81 \mathrm{E}+03$ & $6.70 \mathrm{E}+03$ & $6.56 \mathrm{E}+03$ & $6.48 \mathrm{E}+03$ & $6.46 \mathrm{E}+03$ \\
\hline$U 236$ & $4.65 \mathrm{E}-01$ & $2.08 \mathrm{E}+00$ & $3.51 \mathrm{E}+00$ & $4.12 \mathrm{E}+00$ & $4.28 \mathrm{E}+00$ \\
\hline$U 237$ & $1.48 \mathrm{E}-04$ & $2.64 \mathrm{E}-04$ & $3.59 \mathrm{E}-04$ & $3.91 \mathrm{E}-04$ & $3.98 \mathrm{E}-04$ \\
\hline$U 238$ & $9.51 \mathrm{E}+05$ & $9.35 \mathrm{E}+05$ & $9.15 \mathrm{E}+05$ & $9.05 \mathrm{E}+05$ & $9.02 \mathrm{E}+05$ \\
\hline$N p 236$ & $1.06 \mathrm{E}-13$ & $4.34 \mathrm{E}-12$ & $8.33 \mathrm{E}-12$ & $9.74 \mathrm{E}-12$ & $1.01 \mathrm{E}-11$ \\
\hline$N p 237$ & $1.53 \mathrm{E}-02$ & $5.78 \mathrm{E}-01$ & $1.07 \mathrm{E}+00$ & $1.24 \mathrm{E}+00$ & $1.28 \mathrm{E}+00$ \\
\hline
\end{tabular}




\begin{tabular}{|c|c|c|c|c|c|}
\hline \multicolumn{6}{|c|}{ Isotopic input mass vector } \\
\hline Isotope & $1^{\text {st }}$ Cycle & $2^{\text {nd }}$ Cycle & $5^{\text {th }}$ Cycle & $10^{\text {th }}$ Cycle & $15^{\text {th }}$ Cycle \\
\hline$N p 238$ & $8.81 \mathrm{E}-09$ & $3.18 \mathrm{E}-07$ & $5.47 \mathrm{E}-07$ & $6.12 \mathrm{E}-07$ & $6.25 \mathrm{E}-07$ \\
\hline Np239 & $4.14 \mathrm{E}-04$ & $4.23 \mathrm{E}-04$ & $4.14 \mathrm{E}-04$ & $4.09 \mathrm{E}-04$ & $4.07 \mathrm{E}-04$ \\
\hline Pu236 & $2.97 \mathrm{E}-11$ & $3.31 \mathrm{E}-10$ & $1.62 \mathrm{E}-09$ & $2.43 \mathrm{E}-09$ & $2.63 \mathrm{E}-09$ \\
\hline Pu237 & $4.35 \mathrm{E}-09$ & $1.93 \mathrm{E}-08$ & $8.13 \mathrm{E}-08$ & $1.20 \mathrm{E}-07$ & $1.29 \mathrm{E}-07$ \\
\hline Pu238 & $3.15 \mathrm{E}+01$ & $3.20 \mathrm{E}+02$ & $1.49 \mathrm{E}+03$ & $2.21 \mathrm{E}+03$ & $2.38 \mathrm{E}+03$ \\
\hline Pu239 & $3.97 \mathrm{E}+04$ & $4.17 \mathrm{E}+04$ & $4.70 \mathrm{E}+04$ & $5.00 \mathrm{E}+04$ & $5.10 \mathrm{E}+04$ \\
\hline Pu240 & $2.23 \mathrm{E}+03$ & $1.00 \mathrm{E}+04$ & $1.69 \mathrm{E}+04$ & $1.99 \mathrm{E}+04$ & $2.06 \mathrm{E}+04$ \\
\hline Pu241 & $9.26 \mathrm{E}+01$ & $3.50 \mathrm{E}+03$ & $6.48 \mathrm{E}+03$ & $7.50 \mathrm{E}+03$ & $7.73 \mathrm{E}+03$ \\
\hline Pu242 & $3.65 \mathrm{E}+01$ & $1.77 \mathrm{E}+03$ & $5.37 \mathrm{E}+03$ & $7.85 \mathrm{E}+03$ & $8.73 \mathrm{E}+03$ \\
\hline Pu243 & $2.90 \mathrm{E}-05$ & $1.03 \mathrm{E}-03$ & $2.04 \mathrm{E}-03$ & $2.47 \mathrm{E}-03$ & $2.59 \mathrm{E}-03$ \\
\hline Pu244 & $4.07 \mathrm{E}-12$ & $4.07 \mathrm{E}-12$ & $4.07 \mathrm{E}-12$ & $4.07 \mathrm{E}-12$ & $4.07 \mathrm{E}-12$ \\
\hline$A m 241$ & $9.41 \mathrm{E}+00$ & $3.56 \mathrm{E}+02$ & $6.58 \mathrm{E}+02$ & $7.62 \mathrm{E}+02$ & $7.85 \mathrm{E}+02$ \\
\hline$A m 242$ & $1.10 \mathrm{E}-05$ & $3.66 \mathrm{E}-04$ & $5.88 \mathrm{E}-04$ & $6.41 \mathrm{E}-04$ & $6.50 \mathrm{E}-04$ \\
\hline$A m 242 M$ & $2.41 \mathrm{E}-06$ & $4.46 \mathrm{E}-05$ & $7.15 \mathrm{E}-05$ & $7.80 \mathrm{E}-05$ & $7.91 \mathrm{E}-05$ \\
\hline$A m 243$ & $1.19 \mathrm{E}-06$ & $2.97 \mathrm{E}-06$ & $4.79 \mathrm{E}-06$ & $5.54 \mathrm{E}-06$ & $5.76 \mathrm{E}-06$ \\
\hline $\mathrm{Cm} 242$ & $5.85 \mathrm{E}-08$ & $2.21 \mathrm{E}-07$ & $3.23 \mathrm{E}-07$ & $3.47 \mathrm{E}-07$ & $3.51 \mathrm{E}-07$ \\
\hline $\mathrm{Cm} 243$ & $1.08 \mathrm{E}-06$ & $1.08 \mathrm{E}-06$ & $1.08 \mathrm{E}-06$ & $1.08 \mathrm{E}-06$ & $1.08 \mathrm{E}-06$ \\
\hline $\mathrm{Cm} 244$ & $1.05 \mathrm{E}-06$ & $1.05 \mathrm{E}-06$ & $1.05 \mathrm{E}-06$ & $1.05 \mathrm{E}-06$ & $1.05 \mathrm{E}-06$ \\
\hline $\mathrm{Cm} 245$ & $1.13 \mathrm{E}-06$ & $1.13 \mathrm{E}-06$ & $1.13 \mathrm{E}-06$ & $1.13 \mathrm{E}-06$ & $1.13 \mathrm{E}-06$ \\
\hline $\mathrm{Cm} 246$ & $1.13 \mathrm{E}-06$ & $1.13 \mathrm{E}-06$ & $1.13 \mathrm{E}-06$ & $1.13 \mathrm{E}-06$ & $1.13 \mathrm{E}-06$ \\
\hline $\mathrm{Cm} 247$ & $1.13 \mathrm{E}-06$ & $1.13 \mathrm{E}-06$ & $1.13 \mathrm{E}-06$ & $1.13 \mathrm{E}-06$ & $1.13 \mathrm{E}-06$ \\
\hline $\mathrm{Cm} 248$ & $1.13 \mathrm{E}-06$ & $1.13 \mathrm{E}-06$ & $1.13 \mathrm{E}-06$ & $1.13 \mathrm{E}-06$ & $1.13 \mathrm{E}-06$ \\
\hline
\end{tabular}




\begin{tabular}{|c|c|c|c|c|c|}
\hline \multicolumn{6}{|c|}{ Isotopic input mass vector } \\
\hline Isotope & $1^{\text {st }}$ Cycle & $2^{\text {nd }}$ Cycle & $5^{\text {th }}$ Cycle & $10^{\text {th }}$ Cycle & $15^{\text {th }}$ Cycle \\
\hline Bk249 & $1.41 \mathrm{E}-15$ & $1.47 \mathrm{E}-15$ & $1.46 \mathrm{E}-15$ & $1.45 \mathrm{E}-15$ & $1.44 \mathrm{E}-15$ \\
\hline$C f 249$ & $4.62 \mathrm{E}-21$ & $4.78 \mathrm{E}-21$ & $4.73 \mathrm{E}-21$ & $4.68 \mathrm{E}-21$ & $4.67 \mathrm{E}-21$ \\
\hline$Z n 70$ & $1.26 \mathrm{E}-09$ & $1.26 \mathrm{E}-09$ & $1.27 \mathrm{E}-09$ & $1.27 \mathrm{E}-09$ & $1.28 \mathrm{E}-09$ \\
\hline Ga71 & $5.67 \mathrm{E}-10$ & $5.97 \mathrm{E}-10$ & $6.19 \mathrm{E}-10$ & $6.27 \mathrm{E}-10$ & $6.29 \mathrm{E}-10$ \\
\hline Ge72 & $1.49 \mathrm{E}-12$ & $1.37 \mathrm{E}-12$ & $1.30 \mathrm{E}-12$ & $1.29 \mathrm{E}-12$ & $1.29 \mathrm{E}-12$ \\
\hline Ge73 & $6.36 \mathrm{E}-11$ & $5.96 \mathrm{E}-11$ & $5.75 \mathrm{E}-11$ & $5.71 \mathrm{E}-11$ & $5.70 \mathrm{E}-11$ \\
\hline Ge74 & $4.36 \mathrm{E}-09$ & $4.06 \mathrm{E}-09$ & $3.91 \mathrm{E}-09$ & 3.89E-09 & 3.89E-09 \\
\hline Ge76 & $2.27 \mathrm{E}-07$ & $2.15 \mathrm{E}-07$ & $2.09 \mathrm{E}-07$ & $2.08 \mathrm{E}-07$ & $2.08 \mathrm{E}-07$ \\
\hline$A s 75$ & $1.72 \mathrm{E}-10$ & $1.61 \mathrm{E}-10$ & $1.55 \mathrm{E}-10$ & $1.54 \mathrm{E}-10$ & $1.54 \mathrm{E}-10$ \\
\hline $\mathrm{Se} 77$ & $2.95 \mathrm{E}-11$ & $2.70 \mathrm{E}-11$ & $2.56 \mathrm{E}-11$ & $2.53 \mathrm{E}-11$ & $2.53 \mathrm{E}-11$ \\
\hline $\mathrm{Se} 78$ & $1.96 \mathrm{E}-09$ & $1.83 \mathrm{E}-09$ & $1.76 \mathrm{E}-09$ & $1.74 \mathrm{E}-09$ & $1.74 \mathrm{E}-09$ \\
\hline $\mathrm{Se} 79$ & $4.64 \mathrm{E}-08$ & $4.31 \mathrm{E}-08$ & $4.13 \mathrm{E}-08$ & 4.09E-08 & $4.08 \mathrm{E}-08$ \\
\hline $\mathrm{Se} 80$ & $6.60 \mathrm{E}-06$ & $6.17 \mathrm{E}-06$ & $5.91 \mathrm{E}-06$ & $5.84 \mathrm{E}-06$ & $5.83 \mathrm{E}-06$ \\
\hline $\mathrm{Se} 82$ & $2.58 \mathrm{E}-05$ & $2.46 \mathrm{E}-05$ & $2.39 \mathrm{E}-05$ & $2.38 \mathrm{E}-05$ & $2.37 \mathrm{E}-05$ \\
\hline$B r 79$ & $3.31 \mathrm{E}-11$ & $3.03 \mathrm{E}-11$ & $2.87 \mathrm{E}-11$ & $2.83 \mathrm{E}-11$ & $2.83 \mathrm{E}-11$ \\
\hline$B r 81$ & $2.63 \mathrm{E}-07$ & $2.46 \mathrm{E}-07$ & $2.36 \mathrm{E}-07$ & $2.33 \mathrm{E}-07$ & $2.33 \mathrm{E}-07$ \\
\hline Kr82 & $6.26 \mathrm{E}-10$ & $5.73 \mathrm{E}-10$ & $5.43 \mathrm{E}-10$ & $5.36 \mathrm{E}-10$ & $5.35 \mathrm{E}-10$ \\
\hline Kr83 & $1.46 \mathrm{E}-08$ & $1.33 \mathrm{E}-08$ & $1.25 \mathrm{E}-08$ & $1.24 \mathrm{E}-08$ & $1.23 \mathrm{E}-08$ \\
\hline Kr84 & $9.69 \mathrm{E}-07$ & $9.04 \mathrm{E}-07$ & $8.66 \mathrm{E}-07$ & $8.57 \mathrm{E}-07$ & $8.55 \mathrm{E}-07$ \\
\hline Kr85 & $1.81 \mathrm{E}-06$ & $1.67 \mathrm{E}-06$ & $1.58 \mathrm{E}-06$ & $1.56 \mathrm{E}-06$ & $1.55 \mathrm{E}-06$ \\
\hline Kr86 & $4.82 \mathrm{E}-05$ & $4.64 \mathrm{E}-05$ & $4.52 \mathrm{E}-05$ & $4.48 \mathrm{E}-05$ & $4.47 \mathrm{E}-05$ \\
\hline$R b 85$ & $3.85 \mathrm{E}-08$ & $3.44 \mathrm{E}-08$ & $3.07 \mathrm{E}-08$ & $2.93 \mathrm{E}-08$ & $2.90 \mathrm{E}-08$ \\
\hline
\end{tabular}




\begin{tabular}{|c|c|c|c|c|c|}
\hline \multicolumn{6}{|c|}{ Isotopic input mass vector } \\
\hline Isotope & $1^{\text {st }}$ Cycle & $2^{\text {nd }}$ Cycle & $5^{\text {th }}$ Cycle & $10^{\text {th }}$ Cycle & $15^{\text {th }}$ Cycle \\
\hline$R b 87$ & $2.33 \mathrm{E}-06$ & $2.15 \mathrm{E}-06$ & $2.04 \mathrm{E}-06$ & $2.02 \mathrm{E}-06$ & $2.01 \mathrm{E}-06$ \\
\hline Sr86 & $1.21 \mathrm{E}-10$ & $1.09 \mathrm{E}-10$ & $1.03 \mathrm{E}-10$ & $1.01 \mathrm{E}-10$ & $1.01 \mathrm{E}-10$ \\
\hline Sr87 & $2.98 \mathrm{E}-09$ & $2.71 \mathrm{E}-09$ & $2.55 \mathrm{E}-09$ & $2.51 \mathrm{E}-09$ & $2.51 \mathrm{E}-09$ \\
\hline Sr88 & $3.09 \mathrm{E}-07$ & $2.85 \mathrm{E}-07$ & $2.72 \mathrm{E}-07$ & $2.69 \mathrm{E}-07$ & $2.68 \mathrm{E}-07$ \\
\hline Sr89 & $4.14 \mathrm{E}-06$ & $3.84 \mathrm{E}-06$ & $3.65 \mathrm{E}-06$ & $3.59 \mathrm{E}-06$ & $3.58 \mathrm{E}-06$ \\
\hline $\mathrm{Sr} 90$ & $4.52 \mathrm{E}-05$ & $4.28 \mathrm{E}-05$ & $4.12 \mathrm{E}-05$ & $4.07 \mathrm{E}-05$ & $4.06 \mathrm{E}-05$ \\
\hline$Y 89$ & $8.85 \mathrm{E}-10$ & $8.03 \mathrm{E}-10$ & $7.56 \mathrm{E}-10$ & $7.45 \mathrm{E}-10$ & $7.43 \mathrm{E}-10$ \\
\hline$Y 90$ & $1.21 \mathrm{E}-08$ & $1.10 \mathrm{E}-08$ & $1.04 \mathrm{E}-08$ & $1.02 \mathrm{E}-08$ & $1.02 \mathrm{E}-08$ \\
\hline$Y 91$ & $2.18 \mathrm{E}-07$ & $2.02 \mathrm{E}-07$ & $1.92 \mathrm{E}-07$ & $1.90 \mathrm{E}-07$ & $1.89 \mathrm{E}-07$ \\
\hline $\mathrm{Zr90}$ & $9.76 \mathrm{E}-12$ & $8.87 \mathrm{E}-12$ & $8.36 \mathrm{E}-12$ & $8.24 \mathrm{E}-12$ & $8.22 \mathrm{E}-12$ \\
\hline $\mathrm{Zr91}$ & $4.77 \mathrm{E}-10$ & $4.33 \mathrm{E}-10$ & $4.07 \mathrm{E}-10$ & $4.01 \mathrm{E}-10$ & $4.00 \mathrm{E}-10$ \\
\hline Zr92 & $1.67 \mathrm{E}-07$ & $1.47 \mathrm{E}-07$ & $1.27 \mathrm{E}-07$ & $1.20 \mathrm{E}-07$ & $1.18 \mathrm{E}-07$ \\
\hline $\mathrm{Zr93}$ & $2.63 \mathrm{E}-07$ & $2.41 \mathrm{E}-07$ & $2.28 \mathrm{E}-07$ & $2.25 \mathrm{E}-07$ & $2.25 \mathrm{E}-07$ \\
\hline $\mathrm{Zr94}$ & $1.06 \mathrm{E}-05$ & $9.99 \mathrm{E}-06$ & $9.60 \mathrm{E}-06$ & $9.50 \mathrm{E}-06$ & $9.48 \mathrm{E}-06$ \\
\hline $\mathrm{Zr95}$ & $4.33 \mathrm{E}-05$ & $4.10 \mathrm{E}-05$ & $3.96 \mathrm{E}-05$ & $3.93 \mathrm{E}-05$ & $3.92 \mathrm{E}-05$ \\
\hline $\mathrm{Zr96}$ & $7.32 \mathrm{E}-04$ & $7.22 \mathrm{E}-04$ & $7.15 \mathrm{E}-04$ & $7.12 \mathrm{E}-04$ & $7.12 \mathrm{E}-04$ \\
\hline Nb94 & $1.32 \mathrm{E}-09$ & $1.20 \mathrm{E}-09$ & $1.13 \mathrm{E}-09$ & $1.12 \mathrm{E}-09$ & $1.12 \mathrm{E}-09$ \\
\hline Nb95 & $7.56 \mathrm{E}-08$ & $6.85 \mathrm{E}-08$ & $6.43 \mathrm{E}-08$ & $6.32 \mathrm{E}-08$ & $6.31 \mathrm{E}-08$ \\
\hline Nb95M & $1.76 \mathrm{E}-08$ & $1.59 \mathrm{E}-08$ & $1.50 \mathrm{E}-08$ & $1.47 \mathrm{E}-08$ & $1.47 \mathrm{E}-08$ \\
\hline Mo95 & $3.43 \mathrm{E}-11$ & $3.26 \mathrm{E}-11$ & $3.16 \mathrm{E}-11$ & $3.13 \mathrm{E}-11$ & $3.13 \mathrm{E}-11$ \\
\hline Mo96 & $7.90 \mathrm{E}-10$ & $7.73 \mathrm{E}-10$ & $7.65 \mathrm{E}-10$ & $7.63 \mathrm{E}-10$ & $7.63 \mathrm{E}-10$ \\
\hline Mo97 & $8.59 \mathrm{E}-08$ & $7.83 \mathrm{E}-08$ & 7.39E-08 & $7.28 \mathrm{E}-08$ & $7.26 \mathrm{E}-08$ \\
\hline
\end{tabular}




\begin{tabular}{|c|c|c|c|c|c|}
\hline \multicolumn{6}{|c|}{ Isotopic input mass vector } \\
\hline Isotope & $1^{\text {st }}$ Cycle & $2^{\text {nd }}$ Cycle & $5^{\text {th }}$ Cycle & $10^{\text {th }}$ Cycle & $15^{\text {th }}$ Cycle \\
\hline Mo98 & $5.12 \mathrm{E}-04$ & $5.04 \mathrm{E}-04$ & $4.99 \mathrm{E}-04$ & $4.97 \mathrm{E}-04$ & $4.97 \mathrm{E}-04$ \\
\hline Mo100 & $1.05 \mathrm{E}-03$ & $1.03 \mathrm{E}-03$ & $1.03 \mathrm{E}-03$ & $1.03 \mathrm{E}-03$ & $1.02 \mathrm{E}-03$ \\
\hline Tc98 & $6.76 \mathrm{E}-11$ & $6.09 \mathrm{E}-11$ & $5.66 \mathrm{E}-11$ & $5.54 \mathrm{E}-11$ & $5.51 \mathrm{E}-11$ \\
\hline Tc99 & $1.11 \mathrm{E}-08$ & $1.10 \mathrm{E}-08$ & $1.09 \mathrm{E}-08$ & $1.08 \mathrm{E}-08$ & $1.08 \mathrm{E}-08$ \\
\hline Ru99 & $1.22 \mathrm{E}-13$ & $1.11 \mathrm{E}-13$ & $1.05 \mathrm{E}-13$ & $1.03 \mathrm{E}-13$ & $1.03 \mathrm{E}-13$ \\
\hline Ru100 & $3.24 \mathrm{E}-08$ & $2.94 \mathrm{E}-08$ & $2.78 \mathrm{E}-08$ & $2.74 \mathrm{E}-08$ & $2.73 \mathrm{E}-08$ \\
\hline Ru101 & $6.92 \mathrm{E}-07$ & $6.85 \mathrm{E}-07$ & $6.82 \mathrm{E}-07$ & $6.82 \mathrm{E}-07$ & $6.81 \mathrm{E}-07$ \\
\hline Ru102 & $4.25 \mathrm{E}-05$ & $4.22 \mathrm{E}-05$ & $4.21 \mathrm{E}-05$ & $4.21 \mathrm{E}-05$ & $4.21 \mathrm{E}-05$ \\
\hline Ru103 & $1.40 \mathrm{E}-04$ & $1.39 \mathrm{E}-04$ & $1.39 \mathrm{E}-04$ & $1.39 \mathrm{E}-04$ & $1.39 \mathrm{E}-04$ \\
\hline Ru104 & $1.27 \mathrm{E}-05$ & $1.24 \mathrm{E}-05$ & $1.23 \mathrm{E}-05$ & $1.23 \mathrm{E}-05$ & $1.23 \mathrm{E}-05$ \\
\hline Ru106 & $3.50 \mathrm{E}-04$ & $3.59 \mathrm{E}-04$ & $3.67 \mathrm{E}-04$ & $3.69 \mathrm{E}-04$ & $3.70 \mathrm{E}-04$ \\
\hline Rh103 & $4.92 \mathrm{E}-11$ & $4.86 \mathrm{E}-11$ & $4.84 \mathrm{E}-11$ & $4.83 \mathrm{E}-11$ & $4.83 \mathrm{E}-11$ \\
\hline$R h 103 M$ & $7.71 \mathrm{E}-10$ & $7.60 \mathrm{E}-10$ & $7.55 \mathrm{E}-10$ & $7.55 \mathrm{E}-10$ & $7.55 \mathrm{E}-10$ \\
\hline Rh106 & $1.49 \mathrm{E}-08$ & $1.42 \mathrm{E}-08$ & $1.40 \mathrm{E}-08$ & $1.41 \mathrm{E}-08$ & $1.41 \mathrm{E}-08$ \\
\hline$P d 104$ & $1.55 \mathrm{E}-10$ & $1.42 \mathrm{E}-10$ & $1.34 \mathrm{E}-10$ & $1.32 \mathrm{E}-10$ & $1.32 \mathrm{E}-10$ \\
\hline$P d 105$ & $4.21 \mathrm{E}-11$ & $4.16 \mathrm{E}-11$ & $4.13 \mathrm{E}-11$ & $4.13 \mathrm{E}-11$ & $4.13 \mathrm{E}-11$ \\
\hline$P d 106$ & $2.14 \mathrm{E}-08$ & $2.04 \mathrm{E}-08$ & $2.01 \mathrm{E}-08$ & $2.02 \mathrm{E}-08$ & $2.03 \mathrm{E}-08$ \\
\hline Pd107 & $8.99 \mathrm{E}-07$ & $8.85 \mathrm{E}-07$ & $8.81 \mathrm{E}-07$ & $8.81 \mathrm{E}-07$ & $8.81 \mathrm{E}-07$ \\
\hline$P d 108$ & $2.31 \mathrm{E}-05$ & $2.42 \mathrm{E}-05$ & $2.50 \mathrm{E}-05$ & $2.53 \mathrm{E}-05$ & $2.53 \mathrm{E}-05$ \\
\hline$P d 110$ & $7.97 \mathrm{E}-05$ & $8.75 \mathrm{E}-05$ & $9.30 \mathrm{E}-05$ & $9.45 \mathrm{E}-05$ & $9.48 \mathrm{E}-05$ \\
\hline Ag109 & $3.39 \mathrm{E}-09$ & $3.60 \mathrm{E}-09$ & $3.74 \mathrm{E}-09$ & $3.78 \mathrm{E}-09$ & $3.79 \mathrm{E}-09$ \\
\hline$A g 110 M$ & $4.51 \mathrm{E}-13$ & $6.58 \mathrm{E}-12$ & $1.24 \mathrm{E}-11$ & $1.46 \mathrm{E}-11$ & $1.52 \mathrm{E}-11$ \\
\hline
\end{tabular}




\begin{tabular}{|c|c|c|c|c|c|}
\hline \multicolumn{6}{|c|}{ Isotopic input mass vector } \\
\hline Isotope & $1^{\text {st }}$ Cycle & $2^{\text {nd }}$ Cycle & $5^{\text {th }}$ Cycle & $10^{\text {th }}$ Cycle & $15^{\text {th }}$ Cycle \\
\hline $\operatorname{In} 115$ & $7.80 \mathrm{E}-13$ & $9.42 \mathrm{E}-13$ & $1.44 \mathrm{E}-12$ & $1.73 \mathrm{E}-12$ & $1.80 \mathrm{E}-12$ \\
\hline Sn115 & $1.23 \mathrm{E}-14$ & $1.24 \mathrm{E}-14$ & $1.27 \mathrm{E}-14$ & $1.28 \mathrm{E}-14$ & $1.28 \mathrm{E}-14$ \\
\hline Sn116 & $9.91 \mathrm{E}-12$ & $9.85 \mathrm{E}-12$ & $1.21 \mathrm{E}-11$ & $1.36 \mathrm{E}-11$ & $1.40 \mathrm{E}-11$ \\
\hline Sn117 & $4.69 \mathrm{E}-11$ & $4.45 \mathrm{E}-11$ & $4.45 \mathrm{E}-11$ & $4.52 \mathrm{E}-11$ & $4.54 \mathrm{E}-11$ \\
\hline Sn118 & $7.00 \mathrm{E}-08$ & $6.93 \mathrm{E}-08$ & $6.96 \mathrm{E}-08$ & $7.01 \mathrm{E}-08$ & $7.03 \mathrm{E}-08$ \\
\hline Sn119 & $1.22 \mathrm{E}-07$ & $1.19 \mathrm{E}-07$ & $1.18 \mathrm{E}-07$ & $1.18 \mathrm{E}-07$ & $1.18 \mathrm{E}-07$ \\
\hline Sn120 & $2.92 \mathrm{E}-06$ & $2.94 \mathrm{E}-06$ & $2.96 \mathrm{E}-06$ & $2.98 \mathrm{E}-06$ & $2.98 \mathrm{E}-06$ \\
\hline Sn121 & $3.31 \mathrm{E}-06$ & $3.27 \mathrm{E}-06$ & $3.26 \mathrm{E}-06$ & $3.26 \mathrm{E}-06$ & $3.27 \mathrm{E}-06$ \\
\hline Sn121M & $3.30 \mathrm{E}-07$ & $3.19 \mathrm{E}-07$ & $3.16 \mathrm{E}-07$ & $3.17 \mathrm{E}-07$ & $3.17 \mathrm{E}-07$ \\
\hline Sn122 & $9.15 \mathrm{E}-06$ & $9.01 \mathrm{E}-06$ & $8.97 \mathrm{E}-06$ & $8.98 \mathrm{E}-06$ & 8.99E-06 \\
\hline Sn123 & $3.14 \mathrm{E}-06$ & $3.05 \mathrm{E}-06$ & $3.01 \mathrm{E}-06$ & $3.01 \mathrm{E}-06$ & $3.01 \mathrm{E}-06$ \\
\hline Sn124 & $1.59 \mathrm{E}-05$ & $1.53 \mathrm{E}-05$ & $1.50 \mathrm{E}-05$ & $1.50 \mathrm{E}-05$ & $1.50 \mathrm{E}-05$ \\
\hline Sn126 & $3.92 \mathrm{E}-05$ & $3.76 \mathrm{E}-05$ & $3.68 \mathrm{E}-05$ & $3.67 \mathrm{E}-05$ & $3.67 \mathrm{E}-05$ \\
\hline$S b 121$ & $8.41 \mathrm{E}-10$ & $8.31 \mathrm{E}-10$ & $8.49 \mathrm{E}-10$ & $8.63 \mathrm{E}-10$ & $8.67 \mathrm{E}-10$ \\
\hline$S b 123$ & $1.41 \mathrm{E}-07$ & $1.34 \mathrm{E}-07$ & $1.30 \mathrm{E}-07$ & $1.29 \mathrm{E}-07$ & $1.29 \mathrm{E}-07$ \\
\hline$S b 124$ & $8.75 \mathrm{E}-09$ & $1.01 \mathrm{E}-08$ & $1.10 \mathrm{E}-08$ & $1.13 \mathrm{E}-08$ & $1.13 \mathrm{E}-08$ \\
\hline Sb125 & $5.18 \mathrm{E}-07$ & $4.95 \mathrm{E}-07$ & $4.83 \mathrm{E}-07$ & $4.81 \mathrm{E}-07$ & $4.81 \mathrm{E}-07$ \\
\hline$S b 126$ & $2.64 \mathrm{E}-07$ & $2.43 \mathrm{E}-07$ & $2.32 \mathrm{E}-07$ & $2.29 \mathrm{E}-07$ & $2.29 \mathrm{E}-07$ \\
\hline Te122 & $6.45 \mathrm{E}-12$ & $6.05 \mathrm{E}-12$ & $6.35 \mathrm{E}-12$ & $6.65 \mathrm{E}-12$ & $6.73 \mathrm{E}-12$ \\
\hline Te124 & $8.51 \mathrm{E}-10$ & $9.90 \mathrm{E}-10$ & $1.09 \mathrm{E}-09$ & $1.12 \mathrm{E}-09$ & $1.12 \mathrm{E}-09$ \\
\hline Te125 & $1.82 \mathrm{E}-12$ & $2.36 \mathrm{E}-12$ & $2.92 \mathrm{E}-12$ & $3.18 \mathrm{E}-12$ & $3.25 \mathrm{E}-12$ \\
\hline $\mathrm{Te} 125 \mathrm{M}$ & $6.98 \mathrm{E}-12$ & $1.03 \mathrm{E}-11$ & $1.38 \mathrm{E}-11$ & $1.55 \mathrm{E}-11$ & $1.59 \mathrm{E}-11$ \\
\hline
\end{tabular}




\begin{tabular}{|c|c|c|c|c|c|}
\hline \multicolumn{6}{|c|}{ Isotopic input mass vector } \\
\hline Isotope & $1^{\text {st }}$ Cycle & $2^{\text {nd }}$ Cycle & $5^{\text {th }}$ Cycle & $10^{\text {th }}$ Cycle & $15^{\text {th }}$ Cycle \\
\hline Te126 & $1.05 \mathrm{E}-08$ & $9.62 \mathrm{E}-09$ & $9.13 \mathrm{E}-09$ & $9.02 \mathrm{E}-09$ & $9.01 \mathrm{E}-09$ \\
\hline Te127 & $9.40 \mathrm{E}-10$ & $9.16 \mathrm{E}-10$ & $9.12 \mathrm{E}-10$ & $9.17 \mathrm{E}-10$ & $9.19 \mathrm{E}-10$ \\
\hline $\mathrm{Te} 127 \mathrm{M}$ & $1.07 \mathrm{E}-09$ & $1.20 \mathrm{E}-09$ & $1.31 \mathrm{E}-09$ & $1.36 \mathrm{E}-09$ & $1.38 \mathrm{E}-09$ \\
\hline $\mathrm{Te} 128$ & $5.32 \mathrm{E}-07$ & $4.95 \mathrm{E}-07$ & $4.75 \mathrm{E}-07$ & $4.70 \mathrm{E}-07$ & 4.69E-07 \\
\hline Te129M & $1.14 \mathrm{E}-07$ & $1.10 \mathrm{E}-07$ & $1.12 \mathrm{E}-07$ & $1.14 \mathrm{E}-07$ & $1.14 \mathrm{E}-07$ \\
\hline Te130 & $5.03 \mathrm{E}-05$ & $4.63 \mathrm{E}-05$ & $4.41 \mathrm{E}-05$ & $4.36 \mathrm{E}-05$ & $4.35 \mathrm{E}-05$ \\
\hline$I 127$ & $3.61 \mathrm{E}-12$ & $4.30 \mathrm{E}-12$ & $5.15 \mathrm{E}-12$ & $5.58 \mathrm{E}-12$ & $5.69 \mathrm{E}-12$ \\
\hline$I 129$ & $1.36 \mathrm{E}-09$ & $1.34 \mathrm{E}-09$ & $1.36 \mathrm{E}-09$ & $1.38 \mathrm{E}-09$ & 1.39E-09 \\
\hline Xe128 & $3.16 \mathrm{E}-10$ & $2.85 \mathrm{E}-10$ & $2.67 \mathrm{E}-10$ & $2.62 \mathrm{E}-10$ & $2.61 \mathrm{E}-10$ \\
\hline Xe130 & $3.33 \mathrm{E}-09$ & $3.01 \mathrm{E}-09$ & $2.84 \mathrm{E}-09$ & $2.79 \mathrm{E}-09$ & $2.79 \mathrm{E}-09$ \\
\hline Xe131 & $2.52 \mathrm{E}-09$ & $2.30 \mathrm{E}-09$ & $2.18 \mathrm{E}-09$ & $2.15 \mathrm{E}-09$ & $2.15 \mathrm{E}-09$ \\
\hline Xe132 & $4.01 \mathrm{E}-07$ & $3.68 \mathrm{E}-07$ & $3.49 \mathrm{E}-07$ & $3.45 \mathrm{E}-07$ & $3.44 \mathrm{E}-07$ \\
\hline Xe134 & $5.10 \mathrm{E}-05$ & $4.71 \mathrm{E}-05$ & $4.50 \mathrm{E}-05$ & $4.45 \mathrm{E}-05$ & $4.44 \mathrm{E}-05$ \\
\hline Xe136 & $1.01 \mathrm{E}-03$ & $9.73 \mathrm{E}-04$ & $9.53 \mathrm{E}-04$ & $9.48 \mathrm{E}-04$ & $9.47 \mathrm{E}-04$ \\
\hline Cs133 & $4.00 \mathrm{E}-09$ & $3.65 \mathrm{E}-09$ & $3.46 \mathrm{E}-09$ & $3.42 \mathrm{E}-09$ & $3.42 \mathrm{E}-09$ \\
\hline Cs 134 & $6.33 \mathrm{E}-08$ & $5.79 \mathrm{E}-08$ & $5.50 \mathrm{E}-08$ & 5.44E-08 & $5.43 \mathrm{E}-08$ \\
\hline Cs 135 & $1.28 \mathrm{E}-06$ & $1.17 \mathrm{E}-06$ & $1.11 \mathrm{E}-06$ & $1.09 \mathrm{E}-06$ & $1.09 \mathrm{E}-06$ \\
\hline Cs136 & $9.41 \mathrm{E}-06$ & $8.70 \mathrm{E}-06$ & $8.33 \mathrm{E}-06$ & $8.25 \mathrm{E}-06$ & $8.25 \mathrm{E}-06$ \\
\hline Cs137 & $2.55 \mathrm{E}-04$ & $2.45 \mathrm{E}-04$ & $2.40 \mathrm{E}-04$ & $2.39 \mathrm{E}-04$ & $2.39 \mathrm{E}-04$ \\
\hline Ba134 & $3.83 \mathrm{E}-11$ & $3.53 \mathrm{E}-11$ & $3.40 \mathrm{E}-11$ & $3.38 \mathrm{E}-11$ & $3.39 \mathrm{E}-11$ \\
\hline Ba135 & $1.16 \mathrm{E}-09$ & $1.05 \mathrm{E}-09$ & $9.98 \mathrm{E}-10$ & $9.86 \mathrm{E}-10$ & $9.85 \mathrm{E}-10$ \\
\hline Ba136 & $6.80 \mathrm{E}-06$ & $6.29 \mathrm{E}-06$ & $6.02 \mathrm{E}-06$ & $5.96 \mathrm{E}-06$ & $5.96 \mathrm{E}-06$ \\
\hline
\end{tabular}




\begin{tabular}{|c|c|c|c|c|c|}
\hline \multicolumn{6}{|c|}{ Isotopic input mass vector } \\
\hline Isotope & $1^{\text {st }}$ Cycle & $2^{\text {nd }}$ Cycle & $5^{\text {th }}$ Cycle & $10^{\text {th }}$ Cycle & $15^{\text {th }}$ Cycle \\
\hline Ba137 & $5.13 \mathrm{E}-07$ & $4.67 \mathrm{E}-07$ & $4.42 \mathrm{E}-07$ & $4.36 \mathrm{E}-07$ & 4.35E-07 \\
\hline Ba137M & $7.11 \mathrm{E}-07$ & $6.48 \mathrm{E}-07$ & $6.14 \mathrm{E}-07$ & $6.06 \mathrm{E}-07$ & $6.05 \mathrm{E}-07$ \\
\hline Ba138 & $1.56 \mathrm{E}-05$ & $1.44 \mathrm{E}-05$ & $1.38 \mathrm{E}-05$ & $1.37 \mathrm{E}-05$ & $1.37 \mathrm{E}-05$ \\
\hline Bal40 & $5.97 \mathrm{E}-04$ & $5.77 \mathrm{E}-04$ & $5.67 \mathrm{E}-04$ & $5.64 \mathrm{E}-04$ & $5.63 \mathrm{E}-04$ \\
\hline La138 & $1.24 \mathrm{E}-08$ & $1.13 \mathrm{E}-08$ & $1.07 \mathrm{E}-08$ & $1.05 \mathrm{E}-08$ & $1.05 \mathrm{E}-08$ \\
\hline La139 & $6.62 \mathrm{E}-07$ & $6.18 \mathrm{E}-07$ & $5.94 \mathrm{E}-07$ & $5.89 \mathrm{E}-07$ & $5.88 \mathrm{E}-07$ \\
\hline La140 & $2.11 \mathrm{E}-06$ & $1.92 \mathrm{E}-06$ & $1.82 \mathrm{E}-06$ & $1.80 \mathrm{E}-06$ & $1.79 \mathrm{E}-06$ \\
\hline $\mathrm{Ce} 140$ & $3.04 \mathrm{E}-09$ & $2.76 \mathrm{E}-09$ & 2.61E-09 & $2.58 \mathrm{E}-09$ & $2.58 \mathrm{E}-09$ \\
\hline Cel41 & $1.10 \mathrm{E}-07$ & $1.02 \mathrm{E}-07$ & $9.82 \mathrm{E}-08$ & $9.73 \mathrm{E}-08$ & $9.72 \mathrm{E}-08$ \\
\hline $\mathrm{Ce} 142$ & $1.28 \mathrm{E}-06$ & $1.19 \mathrm{E}-06$ & $1.14 \mathrm{E}-06$ & $1.13 \mathrm{E}-06$ & $1.13 \mathrm{E}-06$ \\
\hline Cel44 & $3.92 \mathrm{E}-04$ & $3.90 \mathrm{E}-04$ & $3.88 \mathrm{E}-04$ & $3.87 \mathrm{E}-04$ & $3.87 \mathrm{E}-04$ \\
\hline $\operatorname{Pr} 141$ & $3.94 \mathrm{E}-11$ & $3.86 \mathrm{E}-11$ & $3.82 \mathrm{E}-11$ & $3.81 \mathrm{E}-11$ & $3.80 \mathrm{E}-11$ \\
\hline $\operatorname{Pr} 143$ & $1.28 \mathrm{E}-08$ & $1.20 \mathrm{E}-08$ & $1.15 \mathrm{E}-08$ & $1.15 \mathrm{E}-08$ & $1.14 \mathrm{E}-08$ \\
\hline $\operatorname{Pr} 144$ & $1.22 \mathrm{E}-08$ & $1.12 \mathrm{E}-08$ & $1.07 \mathrm{E}-08$ & $1.07 \mathrm{E}-08$ & $1.06 \mathrm{E}-08$ \\
\hline$N d 142$ & $5.85 \mathrm{E}-14$ & $5.36 \mathrm{E}-14$ & $5.14 \mathrm{E}-14$ & $5.11 \mathrm{E}-14$ & $5.11 \mathrm{E}-14$ \\
\hline$N d 143$ & $3.06 \mathrm{E}-11$ & $3.04 \mathrm{E}-11$ & $3.03 \mathrm{E}-11$ & $3.03 \mathrm{E}-11$ & $3.03 \mathrm{E}-11$ \\
\hline$N d 144$ & $3.79 \mathrm{E}-10$ & $3.49 \mathrm{E}-10$ & $3.35 \mathrm{E}-10$ & $3.32 \mathrm{E}-10$ & $3.32 \mathrm{E}-10$ \\
\hline$N d 145$ & $5.78 \mathrm{E}-08$ & $5.66 \mathrm{E}-08$ & $5.60 \mathrm{E}-08$ & $5.58 \mathrm{E}-08$ & $5.58 \mathrm{E}-08$ \\
\hline$N d 146$ & $3.85 \mathrm{E}-07$ & $3.76 \mathrm{E}-07$ & $3.71 \mathrm{E}-07$ & $3.70 \mathrm{E}-07$ & $3.70 \mathrm{E}-07$ \\
\hline$N d 147$ & $6.56 \mathrm{E}-06$ & $6.45 \mathrm{E}-06$ & $6.38 \mathrm{E}-06$ & $6.36 \mathrm{E}-06$ & $6.35 \mathrm{E}-06$ \\
\hline$N d 148$ & $4.68 \mathrm{E}-05$ & $4.52 \mathrm{E}-05$ & $4.43 \mathrm{E}-05$ & $4.41 \mathrm{E}-05$ & $4.41 \mathrm{E}-05$ \\
\hline$N d 150$ & $2.12 \mathrm{E}-04$ & $2.15 \mathrm{E}-04$ & $2.17 \mathrm{E}-04$ & $2.18 \mathrm{E}-04$ & $2.18 \mathrm{E}-04$ \\
\hline
\end{tabular}




\begin{tabular}{|c|c|c|c|c|c|}
\hline \multicolumn{6}{|c|}{ Isotopic input mass vector } \\
\hline Isotope & $1^{\text {st }}$ Cycle & $2^{\text {nd }}$ Cycle & $5^{\text {th }}$ Cycle & $10^{\text {th }}$ Cycle & $15^{\text {th }}$ Cycle \\
\hline Pm147 & $2.69 \mathrm{E}-10$ & $2.54 \mathrm{E}-10$ & $2.47 \mathrm{E}-10$ & $2.46 \mathrm{E}-10$ & $2.45 \mathrm{E}-10$ \\
\hline$P m 148$ & $2.74 \mathrm{E}-10$ & $2.55 \mathrm{E}-10$ & $2.51 \mathrm{E}-10$ & $2.52 \mathrm{E}-10$ & $2.53 \mathrm{E}-10$ \\
\hline$P m 148 M$ & $9.64 \mathrm{E}-10$ & $8.92 \mathrm{E}-10$ & $8.69 \mathrm{E}-10$ & $8.71 \mathrm{E}-10$ & $8.73 \mathrm{E}-10$ \\
\hline $\operatorname{Sm} 147$ & $5.83 \mathrm{E}-12$ & $5.86 \mathrm{E}-12$ & $5.89 \mathrm{E}-12$ & $5.89 \mathrm{E}-12$ & $5.89 \mathrm{E}-12$ \\
\hline$S m 148$ & $7.01 \mathrm{E}-13$ & $6.70 \mathrm{E}-13$ & $6.61 \mathrm{E}-13$ & $6.63 \mathrm{E}-13$ & $6.63 \mathrm{E}-13$ \\
\hline Sm 149 & $5.54 \mathrm{E}-11$ & $5.30 \mathrm{E}-11$ & $5.20 \mathrm{E}-11$ & $5.19 \mathrm{E}-11$ & $5.19 \mathrm{E}-11$ \\
\hline$S m 150$ & $1.35 \mathrm{E}-09$ & $1.24 \mathrm{E}-09$ & $1.19 \mathrm{E}-09$ & $1.18 \mathrm{E}-09$ & $1.18 \mathrm{E}-09$ \\
\hline$S m 151$ & $9.98 \mathrm{E}-09$ & $9.20 \mathrm{E}-09$ & $8.83 \mathrm{E}-09$ & 8.77E-09 & 8.77E-09 \\
\hline $\operatorname{Sm} 152$ & $1.16 \mathrm{E}-06$ & $1.11 \mathrm{E}-06$ & $1.09 \mathrm{E}-06$ & $1.09 \mathrm{E}-06$ & $1.08 \mathrm{E}-06$ \\
\hline $\operatorname{Sm} 154$ & $9.73 \mathrm{E}-06$ & $9.69 \mathrm{E}-06$ & $9.72 \mathrm{E}-06$ & $9.74 \mathrm{E}-06$ & $9.75 \mathrm{E}-06$ \\
\hline Eu151 & $1.34 \mathrm{E}-12$ & $1.29 \mathrm{E}-12$ & $1.28 \mathrm{E}-12$ & $1.29 \mathrm{E}-12$ & $1.29 \mathrm{E}-12$ \\
\hline Eu152 & $9.78 \mathrm{E}-12$ & $9.04 \mathrm{E}-12$ & $8.76 \mathrm{E}-12$ & $8.76 \mathrm{E}-12$ & $8.78 \mathrm{E}-12$ \\
\hline Eu153 & $1.13 \mathrm{E}-09$ & $1.08 \mathrm{E}-09$ & $1.07 \mathrm{E}-09$ & $1.07 \mathrm{E}-09$ & $1.07 \mathrm{E}-09$ \\
\hline Eu154 & $3.10 \mathrm{E}-09$ & $2.84 \mathrm{E}-09$ & $2.72 \mathrm{E}-09$ & $2.71 \mathrm{E}-09$ & $2.71 \mathrm{E}-09$ \\
\hline Eu155 & $4.14 \mathrm{E}-07$ & $4.15 \mathrm{E}-07$ & $4.18 \mathrm{E}-07$ & $4.20 \mathrm{E}-07$ & $4.20 \mathrm{E}-07$ \\
\hline Eu156 & $2.79 \mathrm{E}-07$ & $2.58 \mathrm{E}-07$ & $2.48 \mathrm{E}-07$ & $2.46 \mathrm{E}-07$ & $2.46 \mathrm{E}-07$ \\
\hline Gd152 & $3.01 \mathrm{E}-13$ & $3.00 \mathrm{E}-13$ & $3.00 \mathrm{E}-13$ & $3.00 \mathrm{E}-13$ & $3.00 \mathrm{E}-13$ \\
\hline Gd154 & $2.39 \mathrm{E}-12$ & $2.28 \mathrm{E}-12$ & $2.27 \mathrm{E}-12$ & $2.30 \mathrm{E}-12$ & $2.31 \mathrm{E}-12$ \\
\hline$G d 155$ & $3.84 \mathrm{E}-11$ & $3.60 \mathrm{E}-11$ & $3.55 \mathrm{E}-11$ & $3.58 \mathrm{E}-11$ & $3.59 \mathrm{E}-11$ \\
\hline Gd156 & $8.58 \mathrm{E}-10$ & $7.82 \mathrm{E}-10$ & $7.45 \mathrm{E}-10$ & $7.39 \mathrm{E}-10$ & $7.38 \mathrm{E}-10$ \\
\hline Gd157 & $7.37 \mathrm{E}-09$ & $6.84 \mathrm{E}-09$ & $6.60 \mathrm{E}-09$ & $6.58 \mathrm{E}-09$ & $6.58 \mathrm{E}-09$ \\
\hline$G d 158$ & 5.48E-08 & $5.21 \mathrm{E}-08$ & $5.12 \mathrm{E}-08$ & $5.12 \mathrm{E}-08$ & $5.12 \mathrm{E}-08$ \\
\hline
\end{tabular}




\begin{tabular}{|c|c|c|c|c|c|}
\hline \multicolumn{7}{|c|}{ Isotopic input mass vector } \\
\hline Isotope & $\mathbf{1}^{\text {st }}$ Cycle & $\mathbf{2}^{\text {nd }}$ Cycle & $\mathbf{5}^{\text {th }}$ Cycle & $\mathbf{1 0}^{\text {th }}$ Cycle & $\mathbf{1 5}^{\text {th }}$ Cycle \\
\hline Gd160 & $1.18 \mathrm{E}-06$ & $1.27 \mathrm{E}-06$ & $1.35 \mathrm{E}-06$ & $1.37 \mathrm{E}-06$ & $1.37 \mathrm{E}-06$ \\
\hline Tb159 & $3.82 \mathrm{E}-10$ & $3.61 \mathrm{E}-10$ & $3.55 \mathrm{E}-10$ & $3.57 \mathrm{E}-10$ & $3.57 \mathrm{E}-10$ \\
\hline Tb160 & $1.50 \mathrm{E}-09$ & $1.43 \mathrm{E}-09$ & $1.43 \mathrm{E}-09$ & $1.44 \mathrm{E}-09$ & $1.44 \mathrm{E}-09$ \\
\hline Dy160 & $1.13 \mathrm{E}-12$ & $1.08 \mathrm{E}-12$ & $1.10 \mathrm{E}-12$ & $1.12 \mathrm{E}-12$ & $1.13 \mathrm{E}-12$ \\
\hline Dy161 & $1.69 \mathrm{E}-11$ & $1.65 \mathrm{E}-11$ & $1.67 \mathrm{E}-11$ & $1.70 \mathrm{E}-11$ & $1.71 \mathrm{E}-11$ \\
\hline Dy162 & $2.33 \mathrm{E}-09$ & $2.18 \mathrm{E}-09$ & $2.13 \mathrm{E}-09$ & $2.14 \mathrm{E}-09$ & $2.15 \mathrm{E}-09$ \\
\hline Dy163 & $2.40 \mathrm{E}-09$ & $2.26 \mathrm{E}-09$ & $2.22 \mathrm{E}-09$ & $2.24 \mathrm{E}-09$ & $2.24 \mathrm{E}-09$ \\
\hline Dy164 & $8.11 \mathrm{E}-09$ & $7.78 \mathrm{E}-09$ & $7.77 \mathrm{E}-09$ & $7.86 \mathrm{E}-09$ & $7.89 \mathrm{E}-09$ \\
\hline Ho165 & $5.47 \mathrm{E}-11$ & $5.35 \mathrm{E}-11$ & $5.48 \mathrm{E}-11$ & $5.60 \mathrm{E}-11$ & $5.64 \mathrm{E}-11$ \\
\hline Er166 & $1.21 \mathrm{E}-13$ & $1.43 \mathrm{E}-13$ & $1.73 \mathrm{E}-13$ & $1.89 \mathrm{E}-13$ & $1.93 \mathrm{E}-13$ \\
\hline Er167 & $1.47 \mathrm{E}-12$ & $1.60 \mathrm{E}-12$ & $1.81 \mathrm{E}-12$ & $1.92 \mathrm{E}-12$ & $1.96 \mathrm{E}-12$ \\
\hline Er168 & $3.05 \mathrm{E}-11$ & $3.37 \mathrm{E}-11$ & $3.75 \mathrm{E}-11$ & $3.94 \mathrm{E}-11$ & $3.99 \mathrm{E}-11$ \\
\hline & & & & & \\
\hline
\end{tabular}


Appendix 2: Detailed isotopic mass fraction output (discharged burnup $50 \mathrm{GWd} / \mathrm{tHM})$.

\begin{tabular}{|c|c|c|c|c|c|}
\hline \multicolumn{6}{|c|}{ Isotopic output mass vector } \\
\hline Isotope & $1^{\text {st }}$ Cycle & $2^{\text {nd }}$ Cycle & $5^{\text {th }}$ Cycle & $10^{\text {th }}$ Cycle & $15^{\text {th }}$ Cycle \\
\hline IDs & $\mathrm{kg} / \mathrm{tIHM}$ & $\mathrm{kg} / \mathrm{tIHM}$ & $\mathrm{kg} / \mathrm{tIH} M$ & $\mathrm{~kg} / \mathrm{tIH} M$ & $\mathrm{~kg} / \mathrm{tIHM}$ \\
\hline $\mathrm{He} 4$ & $8.68 \mathrm{E}+00$ & $1.53 \mathrm{E}+01$ & $2.06 \mathrm{E}+01$ & $2.24 \mathrm{E}+01$ & $2.28 \mathrm{E}+01$ \\
\hline $\operatorname{Ra} 228$ & $5.29 \mathrm{E}-14$ & $4.50 \mathrm{E}-14$ & $3.83 \mathrm{E}-14$ & $3.59 \mathrm{E}-14$ & $3.53 \mathrm{E}-14$ \\
\hline Th228 & $3.45 \mathrm{E}-06$ & $4.55 \mathrm{E}-06$ & $8.08 \mathrm{E}-06$ & $1.03 \mathrm{E}-05$ & $1.09 \mathrm{E}-05$ \\
\hline Th229 & $9.25 \mathrm{E}-07$ & $1.16 \mathrm{E}-06$ & $2.04 \mathrm{E}-06$ & $2.57 \mathrm{E}-06$ & $2.69 \mathrm{E}-06$ \\
\hline Th230 & $4.02 \mathrm{E}-04$ & 4.84E-04 & $7.47 \mathrm{E}-04$ & $9.10 \mathrm{E}-04$ & $9.51 \mathrm{E}-04$ \\
\hline Th231 & $4.16 \mathrm{E}-07$ & $4.46 \mathrm{E}-07$ & $6.11 \mathrm{E}-07$ & $7.07 \mathrm{E}-07$ & $7.29 \mathrm{E}-07$ \\
\hline Th232 & $6.21 \mathrm{E}-04$ & $5.26 \mathrm{E}-04$ & 4.44E-04 & $4.13 \mathrm{E}-04$ & $4.05 \mathrm{E}-04$ \\
\hline Th233 & 1.19E-09 & $9.44 \mathrm{E}-10$ & $7.58 \mathrm{E}-10$ & $6.94 \mathrm{E}-10$ & $6.77 \mathrm{E}-10$ \\
\hline Pa231 & $1.87 \mathrm{E}-04$ & $2.09 \mathrm{E}-04$ & $2.79 \mathrm{E}-04$ & $3.20 \mathrm{E}-04$ & $3.29 \mathrm{E}-04$ \\
\hline$P a 233$ & $9.84 \mathrm{E}-06$ & $9.50 \mathrm{E}-06$ & $9.15 \mathrm{E}-06$ & $9.01 \mathrm{E}-06$ & $8.97 \mathrm{E}-06$ \\
\hline Pa234 & $1.57 \mathrm{E}-07$ & $1.54 \mathrm{E}-07$ & $1.51 \mathrm{E}-07$ & $1.50 \mathrm{E}-07$ & $1.49 \mathrm{E}-07$ \\
\hline U232 & $5.19 \mathrm{E}-04$ & $6.54 \mathrm{E}-04$ & $1.06 \mathrm{E}-03$ & $1.31 \mathrm{E}-03$ & $1.38 \mathrm{E}-03$ \\
\hline$U 233$ & $1.21 \mathrm{E}-03$ & $1.59 \mathrm{E}-03$ & $2.47 \mathrm{E}-03$ & $3.00 \mathrm{E}-03$ & $3.14 \mathrm{E}-03$ \\
\hline$U 234$ & $3.01 \mathrm{E}+01$ & $4.15 \mathrm{E}+01$ & $7.66 \mathrm{E}+01$ & $9.80 \mathrm{E}+01$ & $1.03 \mathrm{E}+02$ \\
\hline$U 235$ & $2.39 \mathrm{E}+03$ & $2.85 \mathrm{E}+03$ & $3.22 \mathrm{E}+03$ & $3.34 \mathrm{E}+03$ & $3.37 \mathrm{E}+03$ \\
\hline$U 236$ & $8.48 \mathrm{E}+02$ & $7.93 \mathrm{E}+02$ & $7.39 \mathrm{E}+02$ & $7.16 \mathrm{E}+02$ & $7.10 \mathrm{E}+02$ \\
\hline$U 237$ & $3.49 \mathrm{E}+00$ & $3.23 \mathrm{E}+00$ & $3.00 \mathrm{E}+00$ & $2.91 \mathrm{E}+00$ & $2.88 \mathrm{E}+00$ \\
\hline$U 238$ & $9.17 \mathrm{E}+05$ & $9.03 \mathrm{E}+05$ & $8.84 \mathrm{E}+05$ & $8.74 \mathrm{E}+05$ & $8.72 \mathrm{E}+05$ \\
\hline$N p 236$ & $2.58 \mathrm{E}-04$ & $3.26 \mathrm{E}-04$ & $3.97 \mathrm{E}-04$ & $4.27 \mathrm{E}-04$ & $4.35 \mathrm{E}-04$ \\
\hline$N p 236 M$ & $1.10 \mathrm{E}-05$ & $1.09 \mathrm{E}-05$ & $1.08 \mathrm{E}-05$ & $1.07 \mathrm{E}-05$ & $1.07 \mathrm{E}-05$ \\
\hline$N p 237$ & $2.41 \mathrm{E}+02$ & $2.43 \mathrm{E}+02$ & $2.42 \mathrm{E}+02$ & $2.41 \mathrm{E}+02$ & $2.40 \mathrm{E}+02$ \\
\hline$N p 238$ & $5.87 \mathrm{E}-01$ & $5.07 \mathrm{E}-01$ & $4.39 \mathrm{E}-01$ & 4.14E-01 & $4.07 \mathrm{E}-01$ \\
\hline$N p 239$ & $8.38 \mathrm{E}+01$ & $7.69 \mathrm{E}+01$ & $7.15 \mathrm{E}+01$ & $6.94 \mathrm{E}+01$ & $6.89 \mathrm{E}+01$ \\
\hline Pu236 & $1.53 \mathrm{E}-03$ & $1.90 \mathrm{E}-03$ & $2.78 \mathrm{E}-03$ & $3.31 \mathrm{E}-03$ & $3.44 \mathrm{E}-03$ \\
\hline Pu237 & $6.70 \mathrm{E}-04$ & $1.94 \mathrm{E}-03$ & $4.95 \mathrm{E}-03$ & $6.78 \mathrm{E}-03$ & $7.23 \mathrm{E}-03$ \\
\hline Pu238 & $2.39 \mathrm{E}+02$ & $6.67 \mathrm{E}+02$ & $1.62 \mathrm{E}+03$ & $2.17 \mathrm{E}+03$ & $2.31 \mathrm{E}+03$ \\
\hline Pu239 & $1.24 \mathrm{E}+04$ & $1.71 \mathrm{E}+04$ & $2.31 \mathrm{E}+04$ & $2.60 \mathrm{E}+04$ & $2.69 \mathrm{E}+04$ \\
\hline Pu240 & $8.33 \mathrm{E}+03$ & $1.18 \mathrm{E}+04$ & $1.63 \mathrm{E}+04$ & $1.85 \mathrm{E}+04$ & $1.92 \mathrm{E}+04$ \\
\hline Pu241 & $4.82 \mathrm{E}+03$ & $7.04 \mathrm{E}+03$ & $9.50 \mathrm{E}+03$ & $1.06 \mathrm{E}+04$ & $1.08 \mathrm{E}+04$ \\
\hline Pu242 & $1.74 \mathrm{E}+03$ & $3.25 \mathrm{E}+03$ & $6.08 \mathrm{E}+03$ & $8.09 \mathrm{E}+03$ & $8.80 \mathrm{E}+03$ \\
\hline Pu243 & $3.88 \mathrm{E}-01$ & $5.50 \mathrm{E}-01$ & $7.36 \mathrm{E}-01$ & $8.30 \mathrm{E}-01$ & $8.59 \mathrm{E}-01$ \\
\hline Pu244 & $7.80 \mathrm{E}-02$ & $2.68 \mathrm{E}-01$ & $4.25 \mathrm{E}-01$ & $4.84 \mathrm{E}-01$ & $5.00 \mathrm{E}-01$ \\
\hline Am240 & $1.22 \mathrm{E}-05$ & $2.57 \mathrm{E}-05$ & 4.31E-05 & $5.11 \mathrm{E}-05$ & $5.32 \mathrm{E}-05$ \\
\hline
\end{tabular}




\begin{tabular}{|c|c|c|c|c|c|}
\hline \multicolumn{6}{|c|}{ Isotopic output mass vector } \\
\hline Isotope & $1^{\text {st }}$ Cycle & $2^{\text {nd }}$ Cycle & $5^{\text {th }}$ Cycle & $10^{\text {th }}$ Cycle & $15^{\text {th }}$ Cycle \\
\hline Am241 & $2.69 \mathrm{E}+02$ & $5.74 \mathrm{E}+02$ & $9.67 \mathrm{E}+02$ & $1.15 \mathrm{E}+03$ & $1.19 \mathrm{E}+03$ \\
\hline Am242 & 4.70E-01 & $7.74 \mathrm{E}-01$ & $1.03 \mathrm{E}+00$ & $1.11 \mathrm{E}+00$ & $1.13 \mathrm{E}+00$ \\
\hline$A m 242 M$ & $4.63 \mathrm{E}+00$ & $1.15 \mathrm{E}+01$ & $2.17 \mathrm{E}+01$ & $2.67 \mathrm{E}+01$ & $2.80 \mathrm{E}+01$ \\
\hline Am243 & $4.56 \mathrm{E}+02$ & $1.05 \mathrm{E}+03$ & $1.76 \mathrm{E}+03$ & $2.10 \mathrm{E}+03$ & $2.20 \mathrm{E}+03$ \\
\hline Am244 & $2.23 \mathrm{E}-02$ & $4.40 \mathrm{E}-02$ & $6.31 \mathrm{E}-02$ & $7.08 \mathrm{E}-02$ & $7.31 \mathrm{E}-02$ \\
\hline$A m 244 M$ & $1.38 \mathrm{E}-02$ & $2.73 \mathrm{E}-02$ & $3.91 \mathrm{E}-02$ & $4.39 \mathrm{E}-02$ & 4.53E-02 \\
\hline $\mathrm{Cm} 242$ & $7.48 \mathrm{E}+01$ & $1.38 \mathrm{E}+02$ & $1.88 \mathrm{E}+02$ & $2.03 \mathrm{E}+02$ & $2.06 \mathrm{E}+02$ \\
\hline $\mathrm{Cm} 243$ & $2.29 \mathrm{E}+00$ & $5.76 \mathrm{E}+00$ & $8.45 \mathrm{E}+00$ & $9.25 \mathrm{E}+00$ & $9.41 \mathrm{E}+00$ \\
\hline $\mathrm{Cm} 244$ & $1.98 \mathrm{E}+02$ & $6.46 \mathrm{E}+02$ & $1.03 \mathrm{E}+03$ & $1.18 \mathrm{E}+03$ & $1.23 \mathrm{E}+03$ \\
\hline $\mathrm{Cm} 245$ & $1.88 \mathrm{E}+01$ & $8.19 \mathrm{E}+01$ & $1.35 \mathrm{E}+02$ & $1.54 \mathrm{E}+02$ & $1.59 \mathrm{E}+02$ \\
\hline $\mathrm{Cm} 246$ & $1.62 \mathrm{E}+00$ & $7.38 \mathrm{E}+00$ & $9.52 \mathrm{E}+00$ & $9.83 \mathrm{E}+00$ & $9.89 \mathrm{E}+00$ \\
\hline $\mathrm{Cm} 247$ & $2.72 \mathrm{E}-02$ & $1.54 \mathrm{E}-01$ & $1.98 \mathrm{E}-01$ & $2.04 \mathrm{E}-01$ & $2.04 \mathrm{E}-01$ \\
\hline $\mathrm{Cm} 248$ & $2.02 \mathrm{E}-03$ & $1.25 \mathrm{E}-02$ & $1.43 \mathrm{E}-02$ & $1.39 \mathrm{E}-02$ & $1.37 \mathrm{E}-02$ \\
\hline$B k 249$ & $3.14 \mathrm{E}-05$ & $2.28 \mathrm{E}-04$ & $2.73 \mathrm{E}-04$ & $2.69 \mathrm{E}-04$ & $2.66 \mathrm{E}-04$ \\
\hline$C f 249$ & $6.65 \mathrm{E}-06$ & $6.01 \mathrm{E}-05$ & $7.81 \mathrm{E}-05$ & $7.90 \mathrm{E}-05$ & $7.85 \mathrm{E}-05$ \\
\hline$Z n 70$ & 4.16E-03 & $4.18 \mathrm{E}-03$ & $4.22 \mathrm{E}-03$ & $4.24 \mathrm{E}-03$ & 4.25E-03 \\
\hline$Z n 72$ & $3.41 \mathrm{E}-05$ & $3.42 \mathrm{E}-05$ & $3.48 \mathrm{E}-05$ & $3.52 \mathrm{E}-05$ & $3.53 \mathrm{E}-05$ \\
\hline Ga71 & 7.79E-03 & $8.06 \mathrm{E}-03$ & $8.29 \mathrm{E}-03$ & 8.37E-03 & 8.39E-03 \\
\hline Ge72 & $1.94 \mathrm{E}-02$ & $1.90 \mathrm{E}-02$ & $1.90 \mathrm{E}-02$ & $1.91 \mathrm{E}-02$ & $1.92 \mathrm{E}-02$ \\
\hline Ge73 & 3.99E-02 & $3.91 \mathrm{E}-02$ & $3.90 \mathrm{E}-02$ & $3.91 \mathrm{E}-02$ & $3.91 \mathrm{E}-02$ \\
\hline Ge74 & $9.75 \mathrm{E}-02$ & $9.41 \mathrm{E}-02$ & $9.32 \mathrm{E}-02$ & $9.34 \mathrm{E}-02$ & $9.35 \mathrm{E}-02$ \\
\hline Ge76 & $4.51 \mathrm{E}-01$ & 4.37E-01 & 4.33E-01 & 4.33E-01 & 4.33E-01 \\
\hline Ge77 & 4.44E-04 & 4.42E-04 & 4.44E-04 & 4.47E-04 & 4.48E-04 \\
\hline Ge78 & $1.65 \mathrm{E}-04$ & $1.64 \mathrm{E}-04$ & $1.64 \mathrm{E}-04$ & $1.64 \mathrm{E}-04$ & $1.65 \mathrm{E}-04$ \\
\hline Ge80 & $4.71 \mathrm{E}-07$ & 4.63E-07 & $4.59 \mathrm{E}-07$ & $4.58 \mathrm{E}-07$ & $4.58 \mathrm{E}-07$ \\
\hline As 75 & $1.96 \mathrm{E}-01$ & $1.89 \mathrm{E}-01$ & $1.87 \mathrm{E}-01$ & $1.87 \mathrm{E}-01$ & $1.87 \mathrm{E}-01$ \\
\hline As 77 & $1.60 \mathrm{E}-03$ & $1.59 \mathrm{E}-03$ & $1.60 \mathrm{E}-03$ & $1.61 \mathrm{E}-03$ & $1.61 \mathrm{E}-03$ \\
\hline As 78 & $1.78 \mathrm{E}-04$ & $1.78 \mathrm{E}-04$ & $1.78 \mathrm{E}-04$ & $1.78 \mathrm{E}-04$ & $1.78 \mathrm{E}-04$ \\
\hline As 79 & $3.63 \mathrm{E}-05$ & $3.59 \mathrm{E}-05$ & $3.59 \mathrm{E}-05$ & $3.59 \mathrm{E}-05$ & $3.60 \mathrm{E}-05$ \\
\hline As80 & 4.74E-07 & 4.66E-07 & 4.62E-07 & 4.61E-07 & 4.61E-07 \\
\hline As81 & $1.85 \mathrm{E}-06$ & $1.83 \mathrm{E}-06$ & $1.81 \mathrm{E}-06$ & $1.81 \mathrm{E}-06$ & $1.81 \mathrm{E}-06$ \\
\hline As82 & $2.37 \mathrm{E}-07$ & $2.35 \mathrm{E}-07$ & $2.32 \mathrm{E}-07$ & $2.31 \mathrm{E}-07$ & $2.30 \mathrm{E}-07$ \\
\hline As $82 M$ & $2.36 \mathrm{E}-08$ & $2.36 \mathrm{E}-08$ & $2.36 \mathrm{E}-08$ & $2.37 \mathrm{E}-08$ & $2.37 \mathrm{E}-08$ \\
\hline As 83 & $7.05 \mathrm{E}-08$ & $6.96 \mathrm{E}-08$ & $6.88 \mathrm{E}-08$ & $6.85 \mathrm{E}-08$ & $6.85 \mathrm{E}-08$ \\
\hline $\mathrm{Se} 77$ & $1.05 \mathrm{E}+00$ & $1.02 \mathrm{E}+00$ & $1.01 \mathrm{E}+00$ & $1.01 \mathrm{E}+00$ & $1.01 \mathrm{E}+00$ \\
\hline $\mathrm{Se} 78$ & $3.03 \mathrm{E}+00$ & $2.96 \mathrm{E}+00$ & $2.92 \mathrm{E}+00$ & $2.92 \mathrm{E}+00$ & $2.92 \mathrm{E}+00$ \\
\hline $\mathrm{Se} 79$ & $6.66 \mathrm{E}+00$ & $6.43 \mathrm{E}+00$ & $6.32 \mathrm{E}+00$ & $6.31 \mathrm{E}+00$ & $6.31 \mathrm{E}+00$ \\
\hline
\end{tabular}




\begin{tabular}{|c|c|c|c|c|c|}
\hline \multicolumn{6}{|c|}{ Isotopic output mass vector } \\
\hline Isotope & $1^{\text {st }}$ Cycle & $2^{\text {nd }}$ Cycle & $5^{\text {th }}$ Cycle & $10^{\text {th }}$ Cycle & $15^{\text {th }}$ Cycle \\
\hline $\mathrm{Se} 79 \mathrm{M}$ & $1.58 \mathrm{E}-05$ & $1.56 \mathrm{E}-05$ & $1.56 \mathrm{E}-05$ & $1.56 \mathrm{E}-05$ & $1.57 \mathrm{E}-05$ \\
\hline $\mathrm{Se} 80$ & $1.48 \mathrm{E}+01$ & $1.41 \mathrm{E}+01$ & $1.37 \mathrm{E}+01$ & $1.36 \mathrm{E}+01$ & $1.36 \mathrm{E}+01$ \\
\hline Se81 & $3.21 \mathrm{E}-04$ & $3.16 \mathrm{E}-04$ & $3.13 \mathrm{E}-04$ & $3.13 \mathrm{E}-04$ & $3.13 \mathrm{E}-04$ \\
\hline Se81M & $9.98 \mathrm{E}-05$ & $9.80 \mathrm{E}-05$ & $9.73 \mathrm{E}-05$ & $9.73 \mathrm{E}-05$ & $9.73 \mathrm{E}-05$ \\
\hline Se82 & $3.93 \mathrm{E}+01$ & $3.81 \mathrm{E}+01$ & $3.75 \mathrm{E}+01$ & $3.73 \mathrm{E}+01$ & $3.72 \mathrm{E}+01$ \\
\hline $\mathrm{Se} 83$ & $6.84 \mathrm{E}-04$ & $6.77 \mathrm{E}-04$ & $6.72 \mathrm{E}-04$ & $6.70 \mathrm{E}-04$ & $6.70 \mathrm{E}-04$ \\
\hline Se83M & $1.89 \mathrm{E}-06$ & $1.87 \mathrm{E}-06$ & $1.86 \mathrm{E}-06$ & $1.85 \mathrm{E}-06$ & $1.85 \mathrm{E}-06$ \\
\hline $\mathrm{Se} 84$ & $1.32 \mathrm{E}-04$ & $1.31 \mathrm{E}-04$ & $1.29 \mathrm{E}-04$ & $1.29 \mathrm{E}-04$ & $1.29 \mathrm{E}-04$ \\
\hline $\mathrm{Se} 85$ & $5.34 \mathrm{E}-06$ & $5.25 \mathrm{E}-06$ & $5.18 \mathrm{E}-06$ & $5.16 \mathrm{E}-06$ & $5.15 \mathrm{E}-06$ \\
\hline $\mathrm{Se} 86$ & $3.61 \mathrm{E}-07$ & $3.54 \mathrm{E}-07$ & $3.48 \mathrm{E}-07$ & $3.46 \mathrm{E}-07$ & $3.45 \mathrm{E}-07$ \\
\hline $\operatorname{Se} 87$ & $1.26 \mathrm{E}-10$ & $1.23 \mathrm{E}-10$ & $1.21 \mathrm{E}-10$ & $1.20 \mathrm{E}-10$ & $1.20 \mathrm{E}-10$ \\
\hline Br79 & $3.39 \mathrm{E}-03$ & $3.27 \mathrm{E}-03$ & $3.22 \mathrm{E}-03$ & $3.22 \mathrm{E}-03$ & $3.22 \mathrm{E}-03$ \\
\hline Br81 & $2.73 \mathrm{E}+01$ & $2.61 \mathrm{E}+01$ & $2.55 \mathrm{E}+01$ & $2.53 \mathrm{E}+01$ & $2.53 \mathrm{E}+01$ \\
\hline Br82 & $3.46 \mathrm{E}-03$ & $3.16 \mathrm{E}-03$ & $2.97 \mathrm{E}-03$ & $2.91 \mathrm{E}-03$ & $2.90 \mathrm{E}-03$ \\
\hline Br83 & $5.30 \mathrm{E}-03$ & $5.24 \mathrm{E}-03$ & $5.20 \mathrm{E}-03$ & $5.19 \mathrm{E}-03$ & $5.19 \mathrm{E}-03$ \\
\hline Br84 & $1.88 \mathrm{E}-03$ & $1.86 \mathrm{E}-03$ & $1.84 \mathrm{E}-03$ & $1.84 \mathrm{E}-03$ & $1.84 \mathrm{E}-03$ \\
\hline Br84M & $2.23 \mathrm{E}-05$ & $2.20 \mathrm{E}-05$ & $2.20 \mathrm{E}-05$ & $2.20 \mathrm{E}-05$ & $2.20 \mathrm{E}-05$ \\
\hline Br85 & $1.77 \mathrm{E}-04$ & $1.75 \mathrm{E}-04$ & $1.73 \mathrm{E}-04$ & $1.72 \mathrm{E}-04$ & $1.72 \mathrm{E}-04$ \\
\hline Br86 & $3.85 \mathrm{E}-05$ & $3.80 \mathrm{E}-05$ & $3.76 \mathrm{E}-05$ & $3.74 \mathrm{E}-05$ & $3.73 \mathrm{E}-05$ \\
\hline Br87 & $3.79 \mathrm{E}-05$ & $3.74 \mathrm{E}-05$ & $3.70 \mathrm{E}-05$ & $3.68 \mathrm{E}-05$ & $3.67 \mathrm{E}-05$ \\
\hline Br88 & $8.12 \mathrm{E}-07$ & $8.02 \mathrm{E}-07$ & 7.91E-07 & $7.87 \mathrm{E}-07$ & $7.86 \mathrm{E}-07$ \\
\hline Br89 & $1.80 \mathrm{E}-11$ & $1.78 \mathrm{E}-11$ & $1.75 \mathrm{E}-11$ & $1.74 \mathrm{E}-11$ & $1.74 \mathrm{E}-11$ \\
\hline Kr82 & $1.15 \mathrm{E}+00$ & $1.07 \mathrm{E}+00$ & $1.01 \mathrm{E}+00$ & $9.98 \mathrm{E}-01$ & $9.94 \mathrm{E}-01$ \\
\hline Kr83 & $4.49 \mathrm{E}+01$ & $4.54 \mathrm{E}+01$ & $4.58 \mathrm{E}+01$ & $4.60 \mathrm{E}+01$ & $4.60 \mathrm{E}+01$ \\
\hline Kr84 & $1.06 \mathrm{E}+02$ & $1.02 \mathrm{E}+02$ & $9.87 \mathrm{E}+01$ & $9.78 \mathrm{E}+01$ & $9.76 \mathrm{E}+01$ \\
\hline Kr85 & $2.59 \mathrm{E}+01$ & $2.51 \mathrm{E}+01$ & $2.46 \mathrm{E}+01$ & $2.44 \mathrm{E}+01$ & $2.44 \mathrm{E}+01$ \\
\hline Kr85M & $1.93 \mathrm{E}-02$ & $1.90 \mathrm{E}-02$ & $1.88 \mathrm{E}-02$ & $1.88 \mathrm{E}-02$ & $1.87 \mathrm{E}-02$ \\
\hline Kr86 & $1.57 \mathrm{E}+02$ & $1.53 \mathrm{E}+02$ & $1.50 \mathrm{E}+02$ & $1.50 \mathrm{E}+02$ & $1.49 \mathrm{E}+02$ \\
\hline Kr87 & $1.06 \mathrm{E}-02$ & $1.04 \mathrm{E}-02$ & $1.03 \mathrm{E}-02$ & $1.02 \mathrm{E}-02$ & $1.02 \mathrm{E}-02$ \\
\hline Kr88 & $3.07 \mathrm{E}-02$ & $3.03 \mathrm{E}-02$ & $2.99 \mathrm{E}-02$ & $2.97 \mathrm{E}-02$ & $2.97 \mathrm{E}-02$ \\
\hline Kr89 & $5.18 \mathrm{E}-04$ & $5.10 \mathrm{E}-04$ & $5.03 \mathrm{E}-04$ & $5.00 \mathrm{E}-04$ & $5.00 \mathrm{E}-04$ \\
\hline Kr90 & $2.10 \mathrm{E}-05$ & $2.07 \mathrm{E}-05$ & $2.04 \mathrm{E}-05$ & $2.03 \mathrm{E}-05$ & $2.02 \mathrm{E}-05$ \\
\hline Kr91 & $3.46 \mathrm{E}-08$ & $3.41 \mathrm{E}-08$ & $3.36 \mathrm{E}-08$ & $3.34 \mathrm{E}-08$ & $3.33 \mathrm{E}-08$ \\
\hline Kr92 & $7.96 \mathrm{E}-14$ & $7.88 \mathrm{E}-14$ & $7.77 \mathrm{E}-14$ & $7.72 \mathrm{E}-14$ & $7.71 \mathrm{E}-14$ \\
\hline$R b 85$ & $8.82 \mathrm{E}+01$ & $8.55 \mathrm{E}+01$ & $8.37 \mathrm{E}+01$ & $8.31 \mathrm{E}+01$ & $8.30 \mathrm{E}+01$ \\
\hline$R b 87$ & $2.11 \mathrm{E}+02$ & $2.05 \mathrm{E}+02$ & $2.00 \mathrm{E}+02$ & $1.99 \mathrm{E}+02$ & $1.99 \mathrm{E}+02$ \\
\hline$R b 88$ & $3.31 \mathrm{E}-03$ & $3.26 \mathrm{E}-03$ & $3.22 \mathrm{E}-03$ & $3.21 \mathrm{E}-03$ & $3.21 \mathrm{E}-03$ \\
\hline
\end{tabular}




\begin{tabular}{|c|c|c|c|c|c|}
\hline \multicolumn{6}{|c|}{ Isotopic output mass vector } \\
\hline Isotope & $1^{\text {st }}$ Cycle & $2^{\text {nd }}$ Cycle & $5^{\text {th }}$ Cycle & $10^{\text {th }}$ Cycle & $15^{\text {th }}$ Cycle \\
\hline$R b 89$ & $3.65 \mathrm{E}-03$ & $3.60 \mathrm{E}-03$ & $3.55 \mathrm{E}-03$ & $3.53 \mathrm{E}-03$ & $3.53 \mathrm{E}-03$ \\
\hline$R b 90$ & $5.07 \mathrm{E}-04$ & 4.99E-04 & $4.92 \mathrm{E}-04$ & $4.89 \mathrm{E}-04$ & $4.88 \mathrm{E}-04$ \\
\hline$R b 90 M$ & $2.24 \mathrm{E}-04$ & $2.20 \mathrm{E}-04$ & $2.19 \mathrm{E}-04$ & $2.19 \mathrm{E}-04$ & 2.19E-04 \\
\hline$R b 91$ & $1.31 \mathrm{E}-04$ & $1.30 \mathrm{E}-04$ & $1.28 \mathrm{E}-04$ & $1.28 \mathrm{E}-04$ & $1.28 \mathrm{E}-04$ \\
\hline$R b 92$ & $1.24 \mathrm{E}-10$ & $1.23 \mathrm{E}-10$ & $1.22 \mathrm{E}-10$ & $1.21 \mathrm{E}-10$ & $1.21 \mathrm{E}-10$ \\
\hline$R b 93$ & $1.94 \mathrm{E}-09$ & $1.92 \mathrm{E}-09$ & $1.91 \mathrm{E}-09$ & $1.90 \mathrm{E}-09$ & $1.90 \mathrm{E}-09$ \\
\hline$R b 94$ & $2.07 \mathrm{E}-13$ & $2.07 \mathrm{E}-13$ & $2.05 \mathrm{E}-13$ & $2.05 \mathrm{E}-13$ & $2.05 \mathrm{E}-13$ \\
\hline Sr86 & 4.70E-01 & $4.45 \mathrm{E}-01$ & $4.28 \mathrm{E}-01$ & 4.22E-01 & $4.21 \mathrm{E}-01$ \\
\hline $\operatorname{Sr} 87$ & $5.79 \mathrm{E}-03$ & $5.43 \mathrm{E}-03$ & $5.24 \mathrm{E}-03$ & $5.19 \mathrm{E}-03$ & $5.18 \mathrm{E}-03$ \\
\hline Sr88 & $2.81 \mathrm{E}+02$ & $2.73 \mathrm{E}+02$ & $2.67 \mathrm{E}+02$ & $2.65 \mathrm{E}+02$ & $2.64 \mathrm{E}+02$ \\
\hline $\operatorname{Sr} 89$ & $1.81 \mathrm{E}+01$ & $1.77 \mathrm{E}+01$ & $1.75 \mathrm{E}+01$ & $1.74 \mathrm{E}+01$ & $1.74 \mathrm{E}+01$ \\
\hline $\mathrm{Sr} 90$ & $4.36 \mathrm{E}+02$ & $4.22 \mathrm{E}+02$ & $4.13 \mathrm{E}+02$ & $4.10 \mathrm{E}+02$ & $4.09 \mathrm{E}+02$ \\
\hline $\mathrm{Sr} 91$ & $2.06 \mathrm{E}-01$ & $2.03 \mathrm{E}-01$ & $2.01 \mathrm{E}-01$ & $2.01 \mathrm{E}-01$ & $2.01 \mathrm{E}-01$ \\
\hline $\mathrm{Sr} 92$ & 6.69E-02 & $6.62 \mathrm{E}-02$ & $6.57 \mathrm{E}-02$ & $6.55 \mathrm{E}-02$ & $6.55 \mathrm{E}-02$ \\
\hline $\mathrm{Sr} 93$ & $3.38 \mathrm{E}-03$ & $3.35 \mathrm{E}-03$ & 3.33E-03 & $3.33 \mathrm{E}-03$ & $3.33 \mathrm{E}-03$ \\
\hline $\mathrm{Sr} 94$ & $3.17 \mathrm{E}-04$ & $3.15 \mathrm{E}-04$ & $3.13 \mathrm{E}-04$ & $3.13 \mathrm{E}-04$ & $3.13 \mathrm{E}-04$ \\
\hline $\mathrm{Sr} 95$ & $1.87 \mathrm{E}-05$ & $1.86 \mathrm{E}-05$ & $1.85 \mathrm{E}-05$ & $1.85 \mathrm{E}-05$ & $1.85 \mathrm{E}-05$ \\
\hline $\mathrm{Sr} 96$ & $1.77 \mathrm{E}-13$ & $1.76 \mathrm{E}-13$ & $1.76 \mathrm{E}-13$ & $1.75 \mathrm{E}-13$ & $1.75 \mathrm{E}-13$ \\
\hline$Y 89$ & $3.53 \mathrm{E}+02$ & $3.42 \mathrm{E}+02$ & $3.34 \mathrm{E}+02$ & $3.31 \mathrm{E}+02$ & $3.31 \mathrm{E}+02$ \\
\hline$Y 90$ & $1.13 \mathrm{E}-01$ & $1.09 \mathrm{E}-01$ & $1.06 \mathrm{E}-01$ & $1.05 \mathrm{E}-01$ & $1.05 \mathrm{E}-01$ \\
\hline Y91 & $3.03 \mathrm{E}+01$ & $2.99 \mathrm{E}+01$ & $2.95 \mathrm{E}+01$ & $2.94 \mathrm{E}+01$ & $2.94 \mathrm{E}+01$ \\
\hline$Y 91 M$ & $1.02 \mathrm{E}-02$ & $1.00 \mathrm{E}-02$ & $9.93 \mathrm{E}-03$ & $9.89 \mathrm{E}-03$ & $9.88 \mathrm{E}-03$ \\
\hline Y92 & $9.05 \mathrm{E}-02$ & $8.95 \mathrm{E}-02$ & $8.88 \mathrm{E}-02$ & $8.85 \mathrm{E}-02$ & $8.85 \mathrm{E}-02$ \\
\hline Y93 & $3.22 \mathrm{E}-01$ & $3.20 \mathrm{E}-01$ & $3.18 \mathrm{E}-01$ & $3.17 \mathrm{E}-01$ & $3.17 \mathrm{E}-01$ \\
\hline Y94 & $1.08 \mathrm{E}-02$ & $1.07 \mathrm{E}-02$ & $1.07 \mathrm{E}-02$ & $1.07 \mathrm{E}-02$ & $1.07 \mathrm{E}-02$ \\
\hline Y95 & $6.31 \mathrm{E}-03$ & $6.28 \mathrm{E}-03$ & $6.26 \mathrm{E}-03$ & $6.25 \mathrm{E}-03$ & $6.25 \mathrm{E}-03$ \\
\hline Y96 & $1.29 \mathrm{E}-09$ & $1.29 \mathrm{E}-09$ & $1.29 \mathrm{E}-09$ & $1.28 \mathrm{E}-09$ & $1.28 \mathrm{E}-09$ \\
\hline$Y 96 M$ & $1.36 \mathrm{E}-07$ & $1.36 \mathrm{E}-07$ & $1.35 \mathrm{E}-07$ & $1.35 \mathrm{E}-07$ & $1.35 \mathrm{E}-07$ \\
\hline Y97 & $1.04 \mathrm{E}-11$ & $1.04 \mathrm{E}-11$ & $1.03 \mathrm{E}-11$ & $1.03 \mathrm{E}-11$ & $1.03 \mathrm{E}-11$ \\
\hline$Y 97 M$ & $1.15 \mathrm{E}-13$ & $1.15 \mathrm{E}-13$ & $1.15 \mathrm{E}-13$ & $1.15 \mathrm{E}-13$ & $1.15 \mathrm{E}-13$ \\
\hline$Y 98$ & $7.96 \mathrm{E}-14$ & $7.96 \mathrm{E}-14$ & $7.94 \mathrm{E}-14$ & $7.93 \mathrm{E}-14$ & $7.93 \mathrm{E}-14$ \\
\hline$Y 98 M$ & $1.76 \mathrm{E}-13$ & $1.76 \mathrm{E}-13$ & $1.76 \mathrm{E}-13$ & $1.76 \mathrm{E}-13$ & $1.76 \mathrm{E}-13$ \\
\hline Y99 & $2.08 \mathrm{E}-13$ & $2.08 \mathrm{E}-13$ & $2.08 \mathrm{E}-13$ & $2.07 \mathrm{E}-13$ & $2.07 \mathrm{E}-13$ \\
\hline Zr90 & $2.14 \mathrm{E}+01$ & $2.06 \mathrm{E}+01$ & $2.00 \mathrm{E}+01$ & $1.98 \mathrm{E}+01$ & $1.98 \mathrm{E}+01$ \\
\hline Zr91 & $5.04 \mathrm{E}+02$ & $4.90 \mathrm{E}+02$ & $4.80 \mathrm{E}+02$ & $4.77 \mathrm{E}+02$ & $4.77 \mathrm{E}+02$ \\
\hline Zr92 & $6.37 \mathrm{E}+02$ & $6.22 \mathrm{E}+02$ & $6.12 \mathrm{E}+02$ & $6.09 \mathrm{E}+02$ & $6.09 \mathrm{E}+02$ \\
\hline $\mathrm{Zr} 93$ & $7.76 \mathrm{E}+02$ & $7.60 \mathrm{E}+02$ & $7.51 \mathrm{E}+02$ & $7.48 \mathrm{E}+02$ & $7.47 \mathrm{E}+02$ \\
\hline
\end{tabular}




\begin{tabular}{|c|c|c|c|c|c|}
\hline \multicolumn{6}{|c|}{ Isotopic output mass vector } \\
\hline Isotope & $1^{\text {st }}$ Cycle & $2^{\text {nd }}$ Cycle & $5^{\text {th }}$ Cycle & $10^{\text {th }}$ Cycle & $15^{\text {th }}$ Cycle \\
\hline Zr94 & $8.89 \mathrm{E}+02$ & $8.72 \mathrm{E}+02$ & $8.61 \mathrm{E}+02$ & $8.58 \mathrm{E}+02$ & $8.58 \mathrm{E}+02$ \\
\hline $\operatorname{Zr95}$ & $6.20 \mathrm{E}+01$ & $6.15 \mathrm{E}+01$ & $6.12 \mathrm{E}+01$ & $6.11 \mathrm{E}+01$ & $6.11 \mathrm{E}+01$ \\
\hline $\operatorname{Zr} 96$ & $1.03 \mathrm{E}+03$ & $1.02 \mathrm{E}+03$ & $1.01 \mathrm{E}+03$ & $1.01 \mathrm{E}+03$ & $1.01 \mathrm{E}+03$ \\
\hline $\operatorname{Zr97}$ & $7.73 \mathrm{E}-01$ & $7.71 \mathrm{E}-01$ & $7.70 \mathrm{E}-01$ & $7.70 \mathrm{E}-01$ & $7.70 \mathrm{E}-01$ \\
\hline Zr98 & $6.87 \mathrm{E}-05$ & $6.86 \mathrm{E}-05$ & $6.85 \mathrm{E}-05$ & $6.85 \mathrm{E}-05$ & $6.85 \mathrm{E}-05$ \\
\hline Zr99 & $7.98 \mathrm{E}-13$ & $7.98 \mathrm{E}-13$ & $7.98 \mathrm{E}-13$ & $7.98 \mathrm{E}-13$ & $7.98 \mathrm{E}-13$ \\
\hline Zr100 & 3.89E-08 & $3.89 \mathrm{E}-08$ & $3.89 \mathrm{E}-08$ & $3.88 \mathrm{E}-08$ & $3.88 \mathrm{E}-08$ \\
\hline Zr101 & $5.16 \mathrm{E}-13$ & $5.20 \mathrm{E}-13$ & $5.21 \mathrm{E}-13$ & $5.21 \mathrm{E}-13$ & $5.21 \mathrm{E}-13$ \\
\hline $\mathrm{Zr} 102$ & $4.66 \mathrm{E}-13$ & 4.69E-13 & $4.69 \mathrm{E}-13$ & $4.69 \mathrm{E}-13$ & $4.68 \mathrm{E}-13$ \\
\hline Zr103 & $6.20 \mathrm{E}-14$ & $6.27 \mathrm{E}-14$ & $6.27 \mathrm{E}-14$ & $6.26 \mathrm{E}-14$ & $6.25 \mathrm{E}-14$ \\
\hline $\mathrm{Nb94}$ & 3.37E-03 & $3.26 \mathrm{E}-03$ & $3.21 \mathrm{E}-03$ & $3.20 \mathrm{E}-03$ & $3.20 \mathrm{E}-03$ \\
\hline $\mathrm{Nb95}$ & $3.39 \mathrm{E}+01$ & $3.36 \mathrm{E}+01$ & $3.34 \mathrm{E}+01$ & $3.34 \mathrm{E}+01$ & $3.33 \mathrm{E}+01$ \\
\hline Nb95M & $3.78 \mathrm{E}-02$ & $3.75 \mathrm{E}-02$ & $3.73 \mathrm{E}-02$ & $3.73 \mathrm{E}-02$ & $3.73 \mathrm{E}-02$ \\
\hline $\mathrm{Nb96}$ & $3.40 \mathrm{E}-03$ & $3.22 \mathrm{E}-03$ & $3.08 \mathrm{E}-03$ & $3.02 \mathrm{E}-03$ & $3.01 \mathrm{E}-03$ \\
\hline $\mathrm{Nb97}$ & $5.61 \mathrm{E}-02$ & $5.59 \mathrm{E}-02$ & $5.58 \mathrm{E}-02$ & $5.58 \mathrm{E}-02$ & $5.58 \mathrm{E}-02$ \\
\hline $\mathrm{Nb98}$ & $7.06 \mathrm{E}-06$ & $7.04 \mathrm{E}-06$ & $7.03 \mathrm{E}-06$ & 7.03E-06 & $7.03 \mathrm{E}-06$ \\
\hline Nb98M & $2.30 \mathrm{E}-04$ & $2.27 \mathrm{E}-04$ & $2.26 \mathrm{E}-04$ & $2.26 \mathrm{E}-04$ & $2.26 \mathrm{E}-04$ \\
\hline $\mathrm{Nb99}$ & $6.58 \mathrm{E}-06$ & $6.59 \mathrm{E}-06$ & $6.58 \mathrm{E}-06$ & $6.58 \mathrm{E}-06$ & $6.58 \mathrm{E}-06$ \\
\hline Nb99M & $1.64 \mathrm{E}-04$ & $1.62 \mathrm{E}-04$ & $1.62 \mathrm{E}-04$ & $1.62 \mathrm{E}-04$ & $1.62 \mathrm{E}-04$ \\
\hline $\mathrm{Nb} 100$ & $1.04 \mathrm{E}-08$ & $1.04 \mathrm{E}-08$ & $1.04 \mathrm{E}-08$ & $1.04 \mathrm{E}-08$ & $1.04 \mathrm{E}-08$ \\
\hline Nb100M & $1.64 \mathrm{E}-13$ & $1.63 \mathrm{E}-13$ & $1.64 \mathrm{E}-13$ & $1.64 \mathrm{E}-13$ & $1.65 \mathrm{E}-13$ \\
\hline $\mathrm{Nb101}$ & $5.41 \mathrm{E}-08$ & $5.43 \mathrm{E}-08$ & $5.44 \mathrm{E}-08$ & $5.44 \mathrm{E}-08$ & $5.44 \mathrm{E}-08$ \\
\hline $\mathrm{Nb} 102$ & $3.75 \mathrm{E}-13$ & $3.78 \mathrm{E}-13$ & $3.79 \mathrm{E}-13$ & $3.79 \mathrm{E}-13$ & $3.79 \mathrm{E}-13$ \\
\hline $\mathrm{Nb103}$ & $3.99 \mathrm{E}-13$ & $4.03 \mathrm{E}-13$ & $4.05 \mathrm{E}-13$ & $4.05 \mathrm{E}-13$ & $4.05 \mathrm{E}-13$ \\
\hline $\mathrm{Nb104}$ & $1.77 \mathrm{E}-10$ & $1.80 \mathrm{E}-10$ & $1.81 \mathrm{E}-10$ & $1.81 \mathrm{E}-10$ & $1.81 \mathrm{E}-10$ \\
\hline $\mathrm{Nb105}$ & $2.25 \mathrm{E}-13$ & $2.28 \mathrm{E}-13$ & $2.29 \mathrm{E}-13$ & $2.29 \mathrm{E}-13$ & $2.29 \mathrm{E}-13$ \\
\hline Mo95 & $8.31 \mathrm{E}+02$ & $8.20 \mathrm{E}+02$ & $8.15 \mathrm{E}+02$ & $8.13 \mathrm{E}+02$ & $8.13 \mathrm{E}+02$ \\
\hline Mo96 & $5.76 \mathrm{E}+01$ & $5.34 \mathrm{E}+01$ & $5.00 \mathrm{E}+01$ & $4.88 \mathrm{E}+01$ & $4.84 \mathrm{E}+01$ \\
\hline Mo97 & $1.12 \mathrm{E}+03$ & $1.11 \mathrm{E}+03$ & $1.10 \mathrm{E}+03$ & $1.10 \mathrm{E}+03$ & $1.10 \mathrm{E}+03$ \\
\hline Mo98 & $1.20 \mathrm{E}+03$ & $1.19 \mathrm{E}+03$ & $1.19 \mathrm{E}+03$ & $1.18 \mathrm{E}+03$ & $1.18 \mathrm{E}+03$ \\
\hline Mo99 & $3.61 \mathrm{E}+00$ & $3.61 \mathrm{E}+00$ & $3.61 \mathrm{E}+00$ & $3.61 \mathrm{E}+00$ & $3.61 \mathrm{E}+00$ \\
\hline Mo100 & $1.42 \mathrm{E}+03$ & $1.41 \mathrm{E}+03$ & $1.41 \mathrm{E}+03$ & $1.41 \mathrm{E}+03$ & $1.41 \mathrm{E}+03$ \\
\hline Mo101 & $1.27 \mathrm{E}-02$ & $1.27 \mathrm{E}-02$ & $1.28 \mathrm{E}-02$ & $1.28 \mathrm{E}-02$ & $1.28 \mathrm{E}-02$ \\
\hline Mo102 & $9.76 \mathrm{E}-03$ & $9.82 \mathrm{E}-03$ & $9.86 \mathrm{E}-03$ & $9.87 \mathrm{E}-03$ & $9.88 \mathrm{E}-03$ \\
\hline Mo103 & $5.00 \mathrm{E}-04$ & $5.02 \mathrm{E}-04$ & $5.04 \mathrm{E}-04$ & $5.05 \mathrm{E}-04$ & $5.05 \mathrm{E}-04$ \\
\hline Mo104 & $3.55 \mathrm{E}-04$ & $3.59 \mathrm{E}-04$ & $3.61 \mathrm{E}-04$ & $3.62 \mathrm{E}-04$ & $3.62 \mathrm{E}-04$ \\
\hline Mo105 & $8.39 \mathrm{E}-05$ & $8.49 \mathrm{E}-05$ & $8.55 \mathrm{E}-05$ & $8.57 \mathrm{E}-05$ & $8.57 \mathrm{E}-05$ \\
\hline
\end{tabular}




\begin{tabular}{|c|c|c|c|c|c|}
\hline \multicolumn{6}{|c|}{ Isotopic output mass vector } \\
\hline Isotope & $1^{\text {st }}$ Cycle & $2^{\text {nd }}$ Cycle & $5^{\text {th }}$ Cycle & $10^{\text {th }}$ Cycle & $15^{\text {th }}$ Cycle \\
\hline Mo106 & $8.12 \mathrm{E}-08$ & 8.27E-08 & $8.36 \mathrm{E}-08$ & $8.38 \mathrm{E}-08$ & $8.38 \mathrm{E}-08$ \\
\hline Mo107 & $1.55 \mathrm{E}-12$ & $1.59 \mathrm{E}-12$ & $1.62 \mathrm{E}-12$ & $1.62 \mathrm{E}-12$ & $1.62 \mathrm{E}-12$ \\
\hline Tc98 & $1.28 \mathrm{E}-02$ & $1.30 \mathrm{E}-02$ & $1.32 \mathrm{E}-02$ & $1.33 \mathrm{E}-02$ & $1.33 \mathrm{E}-02$ \\
\hline Tc99 & $1.13 \mathrm{E}+03$ & $1.14 \mathrm{E}+03$ & $1.14 \mathrm{E}+03$ & $1.15 \mathrm{E}+03$ & $1.15 \mathrm{E}+03$ \\
\hline$T c 99 M$ & $2.92 \mathrm{E}-01$ & $2.91 \mathrm{E}-01$ & $2.91 \mathrm{E}-01$ & $2.91 \mathrm{E}-01$ & $2.91 \mathrm{E}-01$ \\
\hline Tc101 & $1.30 \mathrm{E}-02$ & $1.31 \mathrm{E}-02$ & $1.31 \mathrm{E}-02$ & $1.31 \mathrm{E}-02$ & $1.31 \mathrm{E}-02$ \\
\hline Tc102 & $7.66 \mathrm{E}-05$ & $7.71 \mathrm{E}-05$ & $7.74 \mathrm{E}-05$ & $7.75 \mathrm{E}-05$ & $7.75 \mathrm{E}-05$ \\
\hline Tc102M & $1.12 \mathrm{E}-05$ & $1.10 \mathrm{E}-05$ & $1.09 \mathrm{E}-05$ & $1.09 \mathrm{E}-05$ & $1.09 \mathrm{E}-05$ \\
\hline Tc103 & 7.04E-04 & 7.07E-04 & $7.10 \mathrm{E}-04$ & $7.11 \mathrm{E}-04$ & $7.12 \mathrm{E}-04$ \\
\hline Tc104 & $1.68 \mathrm{E}-02$ & $1.70 \mathrm{E}-02$ & $1.71 \mathrm{E}-02$ & $1.72 \mathrm{E}-02$ & $1.72 \mathrm{E}-02$ \\
\hline Tc105 & $5.92 \mathrm{E}-03$ & $5.98 \mathrm{E}-03$ & $6.03 \mathrm{E}-03$ & $6.04 \mathrm{E}-03$ & $6.05 \mathrm{E}-03$ \\
\hline Tc106 & $9.72 \mathrm{E}-05$ & 9.89E-05 & $9.99 \mathrm{E}-05$ & $1.00 \mathrm{E}-04$ & $1.00 \mathrm{E}-04$ \\
\hline Tc107 & $1.22 \mathrm{E}-05$ & $1.25 \mathrm{E}-05$ & $1.26 \mathrm{E}-05$ & $1.27 \mathrm{E}-05$ & $1.27 \mathrm{E}-05$ \\
\hline Tc108 & $4.35 \mathrm{E}-10$ & $4.50 \mathrm{E}-10$ & $4.58 \mathrm{E}-10$ & $4.60 \mathrm{E}-10$ & $4.60 \mathrm{E}-10$ \\
\hline Tc109 & $4.03 \mathrm{E}-14$ & 4.16E-14 & $4.22 \mathrm{E}-14$ & $4.23 \mathrm{E}-14$ & $4.23 \mathrm{E}-14$ \\
\hline Ru99 & $4.75 \mathrm{E}-02$ & 4.74E-02 & $4.75 \mathrm{E}-02$ & $4.75 \mathrm{E}-02$ & $4.75 \mathrm{E}-02$ \\
\hline Ru100 & $1.93 \mathrm{E}+02$ & $1.81 \mathrm{E}+02$ & $1.71 \mathrm{E}+02$ & $1.67 \mathrm{E}+02$ & $1.66 \mathrm{E}+02$ \\
\hline Ru101 & $1.22 \mathrm{E}+03$ & $1.23 \mathrm{E}+03$ & $1.23 \mathrm{E}+03$ & $1.23 \mathrm{E}+03$ & $1.23 \mathrm{E}+03$ \\
\hline Ru102 & $1.41 \mathrm{E}+03$ & $1.41 \mathrm{E}+03$ & $1.42 \mathrm{E}+03$ & $1.42 \mathrm{E}+03$ & $1.42 \mathrm{E}+03$ \\
\hline Ru103 & $5.84 \mathrm{E}+01$ & $5.86 \mathrm{E}+01$ & $5.88 \mathrm{E}+01$ & $5.88 \mathrm{E}+01$ & $5.88 \mathrm{E}+01$ \\
\hline Ru104 & $1.31 \mathrm{E}+03$ & $1.33 \mathrm{E}+03$ & $1.34 \mathrm{E}+03$ & $1.34 \mathrm{E}+03$ & $1.34 \mathrm{E}+03$ \\
\hline Ru105 & $2.27 \mathrm{E}-01$ & $2.29 \mathrm{E}-01$ & $2.31 \mathrm{E}-01$ & $2.31 \mathrm{E}-01$ & $2.31 \mathrm{E}-01$ \\
\hline Ru106 & $3.41 \mathrm{E}+02$ & $3.49 \mathrm{E}+02$ & $3.54 \mathrm{E}+02$ & $3.56 \mathrm{E}+02$ & $3.56 \mathrm{E}+02$ \\
\hline Ru107 & $1.70 \mathrm{E}-03$ & $1.74 \mathrm{E}-03$ & $1.76 \mathrm{E}-03$ & $1.77 \mathrm{E}-03$ & $1.77 \mathrm{E}-03$ \\
\hline Ru108 & $1.40 \mathrm{E}-03$ & $1.44 \mathrm{E}-03$ & $1.46 \mathrm{E}-03$ & $1.46 \mathrm{E}-03$ & $1.47 \mathrm{E}-03$ \\
\hline Ru109 & $2.90 \mathrm{E}-05$ & $2.97 \mathrm{E}-05$ & $3.02 \mathrm{E}-05$ & $3.03 \mathrm{E}-05$ & $3.03 \mathrm{E}-05$ \\
\hline Ru110 & $1.99 \mathrm{E}-07$ & $2.06 \mathrm{E}-07$ & $2.10 \mathrm{E}-07$ & $2.11 \mathrm{E}-07$ & $2.11 \mathrm{E}-07$ \\
\hline Ru111 & $5.17 \mathrm{E}-14$ & $5.37 \mathrm{E}-14$ & $5.48 \mathrm{E}-14$ & $5.51 \mathrm{E}-14$ & $5.52 \mathrm{E}-14$ \\
\hline Rh103 & $9.24 \mathrm{E}+02$ & $9.95 \mathrm{E}+02$ & $1.06 \mathrm{E}+03$ & $1.08 \mathrm{E}+03$ & $1.09 \mathrm{E}+03$ \\
\hline Rh103M & $5.73 \mathrm{E}-02$ & $5.75 \mathrm{E}-02$ & $5.77 \mathrm{E}-02$ & $5.78 \mathrm{E}-02$ & $5.78 \mathrm{E}-02$ \\
\hline Rh105 & $1.71 \mathrm{E}+00$ & $1.75 \mathrm{E}+00$ & $1.77 \mathrm{E}+00$ & $1.78 \mathrm{E}+00$ & $1.79 \mathrm{E}+00$ \\
\hline$R h 105 M$ & $1.72 \mathrm{E}-04$ & $1.73 \mathrm{E}-04$ & $1.74 \mathrm{E}-04$ & $1.75 \mathrm{E}-04$ & $1.75 \mathrm{E}-04$ \\
\hline Rh106 & $3.18 \mathrm{E}-04$ & $3.25 \mathrm{E}-04$ & $3.29 \mathrm{E}-04$ & $3.31 \mathrm{E}-04$ & $3.31 \mathrm{E}-04$ \\
\hline Rh106M & $5.85 \mathrm{E}-04$ & 4.59E-04 & $3.72 \mathrm{E}-04$ & $3.44 \mathrm{E}-04$ & $3.37 \mathrm{E}-04$ \\
\hline Rh107 & $1.18 \mathrm{E}-02$ & $1.20 \mathrm{E}-02$ & $1.21 \mathrm{E}-02$ & $1.22 \mathrm{E}-02$ & $1.22 \mathrm{E}-02$ \\
\hline Rh108 & $9.13 \mathrm{E}-05$ & $9.37 \mathrm{E}-05$ & $9.51 \mathrm{E}-05$ & $9.56 \mathrm{E}-05$ & $9.57 \mathrm{E}-05$ \\
\hline Rh108M & $1.14 \mathrm{E}-05$ & $1.16 \mathrm{E}-05$ & $1.18 \mathrm{E}-05$ & $1.19 \mathrm{E}-05$ & $1.20 \mathrm{E}-05$ \\
\hline
\end{tabular}




\begin{tabular}{|c|c|c|c|c|c|}
\hline \multicolumn{6}{|c|}{ Isotopic output mass vector } \\
\hline Isotope & $1^{\text {st }}$ Cycle & $2^{\text {nd }}$ Cycle & $5^{\text {th }}$ Cycle & $10^{\text {th }}$ Cycle & $15^{\text {th }}$ Cycle \\
\hline Rh109 & $2.49 \mathrm{E}-04$ & $2.55 \mathrm{E}-04$ & $2.59 \mathrm{E}-04$ & $2.60 \mathrm{E}-04$ & 2.61E-04 \\
\hline Rh110 & $7.56 \mathrm{E}-08$ & $7.83 \mathrm{E}-08$ & $7.98 \mathrm{E}-08$ & $8.02 \mathrm{E}-08$ & 8.03E-08 \\
\hline$R h 111$ & 8.24E-08 & $8.65 \mathrm{E}-08$ & $8.88 \mathrm{E}-08$ & $8.95 \mathrm{E}-08$ & 8.97E-08 \\
\hline Rh112 & $3.17 \mathrm{E}-13$ & $3.31 \mathrm{E}-13$ & $3.40 \mathrm{E}-13$ & $3.42 \mathrm{E}-13$ & $3.43 \mathrm{E}-13$ \\
\hline$P d 104$ & $4.82 \mathrm{E}+02$ & $4.12 \mathrm{E}+02$ & $3.53 \mathrm{E}+02$ & $3.31 \mathrm{E}+02$ & $3.25 \mathrm{E}+02$ \\
\hline$P d 105$ & $1.06 \mathrm{E}+03$ & $1.09 \mathrm{E}+03$ & $1.11 \mathrm{E}+03$ & $1.12 \mathrm{E}+03$ & $1.12 \mathrm{E}+03$ \\
\hline$P d 106$ & $7.31 \mathrm{E}+02$ & $7.39 \mathrm{E}+02$ & $7.42 \mathrm{E}+02$ & $7.42 \mathrm{E}+02$ & $7.42 \mathrm{E}+02$ \\
\hline$P d 107$ & $6.82 \mathrm{E}+02$ & $7.08 \mathrm{E}+02$ & $7.24 \mathrm{E}+02$ & $7.29 \mathrm{E}+02$ & $7.30 \mathrm{E}+02$ \\
\hline$P d 108$ & $4.74 \mathrm{E}+02$ & $4.99 \mathrm{E}+02$ & $5.13 \mathrm{E}+02$ & $5.18 \mathrm{E}+02$ & $5.18 \mathrm{E}+02$ \\
\hline Pd109 & $2.94 \mathrm{E}-01$ & $2.98 \mathrm{E}-01$ & $3.00 \mathrm{E}-01$ & $3.00 \mathrm{E}-01$ & $3.00 \mathrm{E}-01$ \\
\hline$P d 110$ & $1.56 \mathrm{E}+02$ & $1.68 \mathrm{E}+02$ & $1.74 \mathrm{E}+02$ & $1.76 \mathrm{E}+02$ & $1.76 \mathrm{E}+02$ \\
\hline$P d 111$ & $1.33 \mathrm{E}-03$ & $1.41 \mathrm{E}-03$ & $1.45 \mathrm{E}-03$ & $1.46 \mathrm{E}-03$ & $1.47 \mathrm{E}-03$ \\
\hline$P d 111 M$ & $4.36 \mathrm{E}-05$ & $7.62 \mathrm{E}-05$ & $9.14 \mathrm{E}-05$ & $9.49 \mathrm{E}-05$ & $9.57 \mathrm{E}-05$ \\
\hline Pd112 & $3.24 \mathrm{E}-02$ & $3.48 \mathrm{E}-02$ & $3.61 \mathrm{E}-02$ & $3.65 \mathrm{E}-02$ & $3.66 \mathrm{E}-02$ \\
\hline Pd113 & $1.55 \mathrm{E}-05$ & $1.66 \mathrm{E}-05$ & $1.71 \mathrm{E}-05$ & $1.73 \mathrm{E}-05$ & $1.73 \mathrm{E}-05$ \\
\hline Pd114 & $1.90 \mathrm{E}-05$ & $2.00 \mathrm{E}-05$ & $2.05 \mathrm{E}-05$ & $2.06 \mathrm{E}-05$ & $2.07 \mathrm{E}-05$ \\
\hline Pd115 & $3.77 \mathrm{E}-07$ & $3.90 \mathrm{E}-07$ & $3.98 \mathrm{E}-07$ & $4.00 \mathrm{E}-07$ & $4.01 \mathrm{E}-07$ \\
\hline Pd116 & $1.35 \mathrm{E}-08$ & $1.36 \mathrm{E}-08$ & $1.38 \mathrm{E}-08$ & $1.38 \mathrm{E}-08$ & $1.39 \mathrm{E}-08$ \\
\hline Ag109 & $2.30 \mathrm{E}+02$ & $2.48 \mathrm{E}+02$ & $2.60 \mathrm{E}+02$ & $2.65 \mathrm{E}+02$ & $2.66 \mathrm{E}+02$ \\
\hline Ag110M & $1.91 \mathrm{E}+00$ & $1.94 \mathrm{E}+00$ & $1.94 \mathrm{E}+00$ & $1.93 \mathrm{E}+00$ & $1.92 \mathrm{E}+00$ \\
\hline Ag111 & $6.71 \mathrm{E}-01$ & $7.03 \mathrm{E}-01$ & 7.19E-01 & $7.23 \mathrm{E}-01$ & 7.24E-01 \\
\hline $\mathrm{Ag} 111 \mathrm{M}$ & $6.20 \mathrm{E}-05$ & $6.54 \mathrm{E}-05$ & $6.72 \mathrm{E}-05$ & $6.77 \mathrm{E}-05$ & $6.78 \mathrm{E}-05$ \\
\hline Ag112 & $4.85 \mathrm{E}-03$ & $5.21 \mathrm{E}-03$ & $5.41 \mathrm{E}-03$ & $5.47 \mathrm{E}-03$ & $5.48 \mathrm{E}-03$ \\
\hline Ag113 & $3.72 \mathrm{E}-03$ & $3.99 \mathrm{E}-03$ & 4.12E-03 & $4.16 \mathrm{E}-03$ & 4.17E-03 \\
\hline $\mathrm{Ag} 113 \mathrm{M}$ & $1.66 \mathrm{E}-05$ & $1.78 \mathrm{E}-05$ & $1.84 \mathrm{E}-05$ & $1.85 \mathrm{E}-05$ & $1.86 \mathrm{E}-05$ \\
\hline Ag115 & $1.60 \mathrm{E}-04$ & $1.66 \mathrm{E}-04$ & $1.69 \mathrm{E}-04$ & $1.70 \mathrm{E}-04$ & $1.71 \mathrm{E}-04$ \\
\hline Ag116 & $1.62 \mathrm{E}-05$ & $1.63 \mathrm{E}-05$ & $1.65 \mathrm{E}-05$ & $1.66 \mathrm{E}-05$ & $1.66 \mathrm{E}-05$ \\
\hline Ag117 & $3.70 \mathrm{E}-06$ & $3.74 \mathrm{E}-06$ & $3.77 \mathrm{E}-06$ & $3.79 \mathrm{E}-06$ & $3.80 \mathrm{E}-06$ \\
\hline Cd110 & $1.61 \mathrm{E}+02$ & $1.63 \mathrm{E}+02$ & $1.60 \mathrm{E}+02$ & $1.58 \mathrm{E}+02$ & $1.57 \mathrm{E}+02$ \\
\hline Cd111 & $7.56 \mathrm{E}+01$ & $8.08 \mathrm{E}+01$ & $8.38 \mathrm{E}+01$ & $8.46 \mathrm{E}+01$ & $8.47 \mathrm{E}+01$ \\
\hline Cd112 & $3.60 \mathrm{E}+01$ & $3.82 \mathrm{E}+01$ & $3.94 \mathrm{E}+01$ & $3.98 \mathrm{E}+01$ & $3.99 \mathrm{E}+01$ \\
\hline Cd113 & $3.21 \mathrm{E}-01$ & $5.14 \mathrm{E}-01$ & 7.73E-01 & $9.05 \mathrm{E}-01$ & $9.44 \mathrm{E}-01$ \\
\hline$C d 113 M$ & $4.81 \mathrm{E}-03$ & $7.52 \mathrm{E}-03$ & $1.11 \mathrm{E}-02$ & $1.30 \mathrm{E}-02$ & $1.35 \mathrm{E}-02$ \\
\hline Cd114 & $3.74 \mathrm{E}+01$ & $3.94 \mathrm{E}+01$ & $4.04 \mathrm{E}+01$ & $4.06 \mathrm{E}+01$ & $4.07 \mathrm{E}+01$ \\
\hline Cd115 & $2.70 \mathrm{E}-02$ & $2.79 \mathrm{E}-02$ & $2.85 \mathrm{E}-02$ & $2.86 \mathrm{E}-02$ & $2.87 \mathrm{E}-02$ \\
\hline$C d 115 \mathrm{M}$ & $3.48 \mathrm{E}-02$ & $3.59 \mathrm{E}-02$ & $3.66 \mathrm{E}-02$ & $3.68 \mathrm{E}-02$ & $3.68 \mathrm{E}-02$ \\
\hline Cd116 & $1.24 \mathrm{E}+01$ & $1.22 \mathrm{E}+01$ & $1.22 \mathrm{E}+01$ & $1.23 \mathrm{E}+01$ & $1.23 \mathrm{E}+01$ \\
\hline
\end{tabular}




\begin{tabular}{|c|c|c|c|c|c|}
\hline \multicolumn{6}{|c|}{ Isotopic output mass vector } \\
\hline Isotope & $1^{\text {st }}$ Cycle & $2^{\text {nd }}$ Cycle & $5^{\text {th }}$ Cycle & $10^{\text {th }}$ Cycle & $15^{\text {th }}$ Cycle \\
\hline Cd117 & $1.09 \mathrm{E}-03$ & $1.10 \mathrm{E}-03$ & $1.11 \mathrm{E}-03$ & $1.12 \mathrm{E}-03$ & $1.12 \mathrm{E}-03$ \\
\hline$C d 117 M$ & $3.64 \mathrm{E}-05$ & $3.62 \mathrm{E}-05$ & $3.69 \mathrm{E}-05$ & $3.74 \mathrm{E}-05$ & $3.76 \mathrm{E}-05$ \\
\hline Cd118 & $3.29 \mathrm{E}-04$ & $3.31 \mathrm{E}-04$ & $3.34 \mathrm{E}-04$ & $3.36 \mathrm{E}-04$ & $3.36 \mathrm{E}-04$ \\
\hline Cd119 & $1.04 \mathrm{E}-05$ & $1.04 \mathrm{E}-05$ & $1.05 \mathrm{E}-05$ & $1.06 \mathrm{E}-05$ & $1.06 \mathrm{E}-05$ \\
\hline Cd119M & $1.45 \mathrm{E}-06$ & $1.45 \mathrm{E}-06$ & $1.46 \mathrm{E}-06$ & $1.48 \mathrm{E}-06$ & $1.48 \mathrm{E}-06$ \\
\hline Cd120 & $1.76 \mathrm{E}-06$ & $1.77 \mathrm{E}-06$ & $1.79 \mathrm{E}-06$ & $1.79 \mathrm{E}-06$ & $1.80 \mathrm{E}-06$ \\
\hline $\operatorname{In} 115$ & $3.41 \mathrm{E}+00$ & $3.91 \mathrm{E}+00$ & $4.40 \mathrm{E}+00$ & $4.62 \mathrm{E}+00$ & $4.68 \mathrm{E}+00$ \\
\hline $\operatorname{In} 117$ & $1.94 \mathrm{E}-04$ & $1.95 \mathrm{E}-04$ & $1.98 \mathrm{E}-04$ & $1.99 \mathrm{E}-04$ & $1.99 \mathrm{E}-04$ \\
\hline $\operatorname{In} 117 M$ & $6.62 \mathrm{E}-04$ & $6.67 \mathrm{E}-04$ & $6.74 \mathrm{E}-04$ & $6.78 \mathrm{E}-04$ & $6.79 \mathrm{E}-04$ \\
\hline $\operatorname{In} 119$ & 7.17E-06 & $7.19 \mathrm{E}-06$ & $7.26 \mathrm{E}-06$ & $7.30 \mathrm{E}-06$ & 7.31E-06 \\
\hline $\operatorname{In} 119 M$ & $6.26 \mathrm{E}-05$ & $6.29 \mathrm{E}-05$ & $6.33 \mathrm{E}-05$ & $6.36 \mathrm{E}-05$ & $6.37 \mathrm{E}-05$ \\
\hline $\operatorname{In} 121$ & $3.01 \mathrm{E}-07$ & $3.01 \mathrm{E}-07$ & $3.03 \mathrm{E}-07$ & $3.05 \mathrm{E}-07$ & $3.05 \mathrm{E}-07$ \\
\hline $\operatorname{In} 121 \mathrm{M}$ & $6.99 \mathrm{E}-06$ & $6.99 \mathrm{E}-06$ & $7.02 \mathrm{E}-06$ & 7.04E-06 & 7.04E-06 \\
\hline Sn115 & 5.39E-01 & $5.54 \mathrm{E}-01$ & $5.65 \mathrm{E}-01$ & $5.70 \mathrm{E}-01$ & $5.71 \mathrm{E}-01$ \\
\hline Sn116 & $4.58 \mathrm{E}+00$ & $4.41 \mathrm{E}+00$ & $4.21 \mathrm{E}+00$ & $4.13 \mathrm{E}+00$ & $4.11 \mathrm{E}+00$ \\
\hline Sn117 & $1.10 \mathrm{E}+01$ & $1.09 \mathrm{E}+01$ & $1.09 \mathrm{E}+01$ & $1.09 \mathrm{E}+01$ & $1.09 \mathrm{E}+01$ \\
\hline Sn118 & $9.63 \mathrm{E}+00$ & $9.62 \mathrm{E}+00$ & $9.68 \mathrm{E}+00$ & $9.72 \mathrm{E}+00$ & $9.74 \mathrm{E}+00$ \\
\hline Sn119 & $9.24 \mathrm{E}+00$ & $9.24 \mathrm{E}+00$ & $9.30 \mathrm{E}+00$ & $9.34 \mathrm{E}+00$ & $9.35 \mathrm{E}+00$ \\
\hline Sn 120 & $9.28 \mathrm{E}+00$ & $9.33 \mathrm{E}+00$ & $9.41 \mathrm{E}+00$ & $9.46 \mathrm{E}+00$ & $9.47 \mathrm{E}+00$ \\
\hline Sn 121 & $1.14 \mathrm{E}-02$ & $1.15 \mathrm{E}-02$ & $1.15 \mathrm{E}-02$ & $1.16 \mathrm{E}-02$ & $1.16 \mathrm{E}-02$ \\
\hline Sn $121 M$ & $6.05 \mathrm{E}-01$ & $6.00 \mathrm{E}-01$ & $6.05 \mathrm{E}-01$ & $6.08 \mathrm{E}-01$ & $6.10 \mathrm{E}-01$ \\
\hline Sn122 & $1.23 \mathrm{E}+01$ & $1.22 \mathrm{E}+01$ & $1.22 \mathrm{E}+01$ & $1.23 \mathrm{E}+01$ & $1.23 \mathrm{E}+01$ \\
\hline Sn123 & 7.13E-01 & 7.13E-01 & 7.17E-01 & $7.20 \mathrm{E}-01$ & $7.21 \mathrm{E}-01$ \\
\hline Sn123M & $1.89 \mathrm{E}-04$ & $1.89 \mathrm{E}-04$ & $1.91 \mathrm{E}-04$ & $1.92 \mathrm{E}-04$ & $1.93 \mathrm{E}-04$ \\
\hline Sn124 & $1.99 \mathrm{E}+01$ & $1.95 \mathrm{E}+01$ & $1.94 \mathrm{E}+01$ & $1.94 \mathrm{E}+01$ & $1.94 \mathrm{E}+01$ \\
\hline Sn $125 M$ & $5.35 \mathrm{E}-05$ & 5.32E-05 & 5.32E-05 & $5.33 \mathrm{E}-05$ & 5.34E-05 \\
\hline Sn126 & $4.70 \mathrm{E}+01$ & $4.58 \mathrm{E}+01$ & $4.55 \mathrm{E}+01$ & $4.55 \mathrm{E}+01$ & $4.55 \mathrm{E}+01$ \\
\hline Sn127 & $6.79 \mathrm{E}-03$ & $6.77 \mathrm{E}-03$ & $6.79 \mathrm{E}-03$ & $6.80 \mathrm{E}-03$ & $6.81 \mathrm{E}-03$ \\
\hline Sn127M & $5.83 \mathrm{E}-05$ & $5.81 \mathrm{E}-05$ & $5.83 \mathrm{E}-05$ & $5.85 \mathrm{E}-05$ & $5.85 \mathrm{E}-05$ \\
\hline Sn 128 & $5.79 \mathrm{E}-03$ & $5.79 \mathrm{E}-03$ & $5.81 \mathrm{E}-03$ & $5.83 \mathrm{E}-03$ & $5.84 \mathrm{E}-03$ \\
\hline Sn129 & $1.82 \mathrm{E}-04$ & $1.82 \mathrm{E}-04$ & $1.83 \mathrm{E}-04$ & $1.84 \mathrm{E}-04$ & $1.84 \mathrm{E}-04$ \\
\hline Sn129M & $2.98 \mathrm{E}-04$ & $2.98 \mathrm{E}-04$ & $2.98 \mathrm{E}-04$ & $2.98 \mathrm{E}-04$ & $2.99 \mathrm{E}-04$ \\
\hline Sn130 & $3.27 \mathrm{E}-04$ & $3.28 \mathrm{E}-04$ & $3.28 \mathrm{E}-04$ & $3.28 \mathrm{E}-04$ & $3.28 \mathrm{E}-04$ \\
\hline Sn131 & $3.12 \mathrm{E}-05$ & $3.14 \mathrm{E}-05$ & $3.14 \mathrm{E}-05$ & $3.14 \mathrm{E}-05$ & $3.14 \mathrm{E}-05$ \\
\hline Sn132 & $1.99 \mathrm{E}-05$ & $1.97 \mathrm{E}-05$ & $1.96 \mathrm{E}-05$ & $1.95 \mathrm{E}-05$ & $1.95 \mathrm{E}-05$ \\
\hline$S b 121$ & $9.22 \mathrm{E}+00$ & $9.23 \mathrm{E}+00$ & $9.31 \mathrm{E}+00$ & $9.36 \mathrm{E}+00$ & $9.37 \mathrm{E}+00$ \\
\hline Sb122 & 5.39E-03 & $5.04 \mathrm{E}-03$ & 4.78E-03 & $4.69 \mathrm{E}-03$ & 4.67E-03 \\
\hline
\end{tabular}




\begin{tabular}{|c|c|c|c|c|c|}
\hline \multicolumn{6}{|c|}{ Isotopic output mass vector } \\
\hline Isotope & $1^{\text {st }}$ Cycle & $2^{\text {nd }}$ Cycle & $5^{\text {th }}$ Cycle & $10^{\text {th }}$ Cycle & $15^{\text {th }}$ Cycle \\
\hline$S b 123$ & $1.12 \mathrm{E}+01$ & $1.12 \mathrm{E}+01$ & $1.13 \mathrm{E}+01$ & $1.13 \mathrm{E}+01$ & $1.13 \mathrm{E}+01$ \\
\hline$S b 124$ & $8.27 \mathrm{E}-02$ & $7.76 \mathrm{E}-02$ & $7.40 \mathrm{E}-02$ & $7.27 \mathrm{E}-02$ & $7.24 \mathrm{E}-02$ \\
\hline$S b 125$ & $1.73 \mathrm{E}+01$ & $1.69 \mathrm{E}+01$ & $1.69 \mathrm{E}+01$ & $1.69 \mathrm{E}+01$ & $1.69 \mathrm{E}+01$ \\
\hline$S b 126$ & $5.89 \mathrm{E}-03$ & $5.72 \mathrm{E}-03$ & $5.64 \mathrm{E}-03$ & $5.63 \mathrm{E}-03$ & $5.63 \mathrm{E}-03$ \\
\hline$S b 127$ & 4.17E-01 & $4.16 \mathrm{E}-01$ & 4.17E-01 & $4.18 \mathrm{E}-01$ & $4.18 \mathrm{E}-01$ \\
\hline$S b 128$ & $5.82 \mathrm{E}-02$ & $5.81 \mathrm{E}-02$ & $5.83 \mathrm{E}-02$ & $5.84 \mathrm{E}-02$ & $5.85 \mathrm{E}-02$ \\
\hline Sb128M & 7.09E-05 & $7.04 \mathrm{E}-05$ & $7.04 \mathrm{E}-05$ & $7.06 \mathrm{E}-05$ & $7.07 \mathrm{E}-05$ \\
\hline$S b 129$ & $5.91 \mathrm{E}-02$ & $5.91 \mathrm{E}-02$ & $5.93 \mathrm{E}-02$ & $5.94 \mathrm{E}-02$ & $5.95 \mathrm{E}-02$ \\
\hline$S b 130$ & $7.68 \mathrm{E}-03$ & $7.68 \mathrm{E}-03$ & 7.69E-03 & $7.69 \mathrm{E}-03$ & 7.69E-03 \\
\hline Sb130M & $1.15 \mathrm{E}-03$ & $1.15 \mathrm{E}-03$ & $1.16 \mathrm{E}-03$ & $1.16 \mathrm{E}-03$ & $1.16 \mathrm{E}-03$ \\
\hline$S b 131$ & $1.17 \mathrm{E}-02$ & $1.17 \mathrm{E}-02$ & $1.18 \mathrm{E}-02$ & $1.18 \mathrm{E}-02$ & $1.18 \mathrm{E}-02$ \\
\hline$S b 132$ & $9.56 \mathrm{E}-04$ & $9.52 \mathrm{E}-04$ & $9.48 \mathrm{E}-04$ & $9.47 \mathrm{E}-04$ & $9.47 \mathrm{E}-04$ \\
\hline Sb132M & $4.98 \mathrm{E}-04$ & $5.04 \mathrm{E}-04$ & $5.07 \mathrm{E}-04$ & $5.07 \mathrm{E}-04$ & $5.07 \mathrm{E}-04$ \\
\hline$S b 133$ & $6.38 \mathrm{E}-04$ & $6.43 \mathrm{E}-04$ & $6.43 \mathrm{E}-04$ & $6.42 \mathrm{E}-04$ & $6.41 \mathrm{E}-04$ \\
\hline Sb134M & $5.16 \mathrm{E}-08$ & $5.18 \mathrm{E}-08$ & $5.17 \mathrm{E}-08$ & $5.15 \mathrm{E}-08$ & $5.15 \mathrm{E}-08$ \\
\hline Te122 & 8.68E-01 & $8.29 \mathrm{E}-01$ & 7.97E-01 & $7.86 \mathrm{E}-01$ & $7.83 \mathrm{E}-01$ \\
\hline Te124 & $5.97 \mathrm{E}-01$ & $5.72 \mathrm{E}-01$ & $5.52 \mathrm{E}-01$ & $5.44 \mathrm{E}-01$ & $5.42 \mathrm{E}-01$ \\
\hline Te125 & $9.72 \mathrm{E}+00$ & $9.43 \mathrm{E}+00$ & $9.34 \mathrm{E}+00$ & $9.34 \mathrm{E}+00$ & $9.34 \mathrm{E}+00$ \\
\hline $\mathrm{Te} 125 \mathrm{M}$ & $2.21 \mathrm{E}-01$ & $2.16 \mathrm{E}-01$ & $2.15 \mathrm{E}-01$ & $2.15 \mathrm{E}-01$ & $2.15 \mathrm{E}-01$ \\
\hline Te126 & $1.01 \mathrm{E}+00$ & $9.54 \mathrm{E}-01$ & $9.25 \mathrm{E}-01$ & $9.19 \mathrm{E}-01$ & $9.18 \mathrm{E}-01$ \\
\hline Te127 & 4.14E-02 & 4.14E-02 & $4.16 \mathrm{E}-02$ & $4.18 \mathrm{E}-02$ & $4.18 \mathrm{E}-02$ \\
\hline $\mathrm{Te} 127 \mathrm{M}$ & $3.59 \mathrm{E}-01$ & $4.03 \mathrm{E}-01$ & $4.41 \mathrm{E}-01$ & $4.54 \mathrm{E}-01$ & $4.58 \mathrm{E}-01$ \\
\hline Te128 & $1.79 \mathrm{E}+02$ & $1.74 \mathrm{E}+02$ & $1.72 \mathrm{E}+02$ & $1.72 \mathrm{E}+02$ & $1.72 \mathrm{E}+02$ \\
\hline $\mathrm{Te} 129 \mathrm{M}$ & $6.66 \mathrm{E}-03$ & $6.68 \mathrm{E}-03$ & $6.86 \mathrm{E}-03$ & $6.97 \mathrm{E}-03$ & $7.00 \mathrm{E}-03$ \\
\hline Te130 & $6.20 \mathrm{E}+02$ & $6.12 \mathrm{E}+02$ & $6.08 \mathrm{E}+02$ & $6.08 \mathrm{E}+02$ & $6.08 \mathrm{E}+02$ \\
\hline Te131 & $1.51 \mathrm{E}-02$ & $1.51 \mathrm{E}-02$ & $1.52 \mathrm{E}-02$ & $1.52 \mathrm{E}-02$ & $1.52 \mathrm{E}-02$ \\
\hline $\mathrm{Te} 131 \mathrm{M}$ & $2.03 \mathrm{E}-01$ & $2.01 \mathrm{E}-01$ & $2.00 \mathrm{E}-01$ & $2.01 \mathrm{E}-01$ & $2.01 \mathrm{E}-01$ \\
\hline Te132 & $4.48 \mathrm{E}+00$ & $4.48 \mathrm{E}+00$ & $4.48 \mathrm{E}+00$ & $4.49 \mathrm{E}+00$ & $4.49 \mathrm{E}+00$ \\
\hline Te133 & $9.20 \mathrm{E}-03$ & $9.22 \mathrm{E}-03$ & $9.22 \mathrm{E}-03$ & $9.21 \mathrm{E}-03$ & $9.21 \mathrm{E}-03$ \\
\hline $\mathrm{Te} 133 \mathrm{M}$ & $3.04 \mathrm{E}-02$ & $3.05 \mathrm{E}-02$ & $3.05 \mathrm{E}-02$ & $3.05 \mathrm{E}-02$ & $3.05 \mathrm{E}-02$ \\
\hline Te134 & $4.52 \mathrm{E}-02$ & $4.53 \mathrm{E}-02$ & $4.53 \mathrm{E}-02$ & $4.53 \mathrm{E}-02$ & $4.52 \mathrm{E}-02$ \\
\hline Te135 & $1.04 \mathrm{E}-05$ & $1.04 \mathrm{E}-05$ & $1.04 \mathrm{E}-05$ & $1.04 \mathrm{E}-05$ & $1.04 \mathrm{E}-05$ \\
\hline Te136 & $2.89 \mathrm{E}-06$ & $2.92 \mathrm{E}-06$ & $2.91 \mathrm{E}-06$ & $2.91 \mathrm{E}-06$ & $2.90 \mathrm{E}-06$ \\
\hline Te137 & $8.76 \mathrm{E}-14$ & $8.78 \mathrm{E}-14$ & $8.72 \mathrm{E}-14$ & $8.68 \mathrm{E}-14$ & 8.67E-14 \\
\hline$I 127$ & $1.06 \mathrm{E}+02$ & $1.04 \mathrm{E}+02$ & $1.03 \mathrm{E}+02$ & $1.03 \mathrm{E}+02$ & $1.03 \mathrm{E}+02$ \\
\hline 1128 & $3.72 \mathrm{E}-04$ & $3.44 \mathrm{E}-04$ & $3.27 \mathrm{E}-04$ & $3.21 \mathrm{E}-04$ & $3.20 \mathrm{E}-04$ \\
\hline 1129 & $3.27 \mathrm{E}+02$ & $3.23 \mathrm{E}+02$ & $3.22 \mathrm{E}+02$ & $3.22 \mathrm{E}+02$ & $3.23 \mathrm{E}+02$ \\
\hline
\end{tabular}




\begin{tabular}{|c|c|c|c|c|c|}
\hline \multicolumn{6}{|c|}{ Isotopic output mass vector } \\
\hline Isotope & $1^{\text {st }}$ Cycle & $2^{\text {nd }}$ Cycle & $5^{\text {th }}$ Cycle & $10^{\text {th }}$ Cycle & $15^{\text {th }}$ Cycle \\
\hline$I 130$ & $1.33 \mathrm{E}-02$ & $1.06 \mathrm{E}-02$ & $8.86 \mathrm{E}-03$ & $8.34 \mathrm{E}-03$ & $8.22 \mathrm{E}-03$ \\
\hline$I 130 M$ & $9.14 \mathrm{E}-05$ & $7.23 \mathrm{E}-05$ & $6.03 \mathrm{E}-05$ & $5.67 \mathrm{E}-05$ & $5.58 \mathrm{E}-05$ \\
\hline$I 132$ & $1.38 \mathrm{E}-01$ & $1.38 \mathrm{E}-01$ & $1.38 \mathrm{E}-01$ & $1.38 \mathrm{E}-01$ & $1.38 \mathrm{E}-01$ \\
\hline$I 133$ & $1.70 \mathrm{E}+00$ & $1.70 \mathrm{E}+00$ & $1.70 \mathrm{E}+00$ & $1.70 \mathrm{E}+00$ & $1.70 \mathrm{E}+00$ \\
\hline$I 133 M$ & $2.23 \mathrm{E}-08$ & $2.20 \mathrm{E}-08$ & $2.20 \mathrm{E}-08$ & $2.21 \mathrm{E}-08$ & $2.21 \mathrm{E}-08$ \\
\hline$I 134$ & 7.89E-02 & 7.91E-02 & $7.91 \mathrm{E}-02$ & $7.91 \mathrm{E}-02$ & 7.91E-02 \\
\hline$I 134 M$ & 4.94E-04 & 4.92E-04 & $4.94 \mathrm{E}-04$ & $4.95 \mathrm{E}-04$ & $4.95 \mathrm{E}-04$ \\
\hline$I 135$ & $5.26 \mathrm{E}-01$ & $5.27 \mathrm{E}-01$ & $5.28 \mathrm{E}-01$ & $5.28 \mathrm{E}-01$ & $5.28 \mathrm{E}-01$ \\
\hline$I 136$ & $4.15 \mathrm{E}-04$ & 4.19E-04 & $4.20 \mathrm{E}-04$ & $4.20 \mathrm{E}-04$ & 4.20E-04 \\
\hline$I 136 M$ & $8.25 \mathrm{E}-05$ & $8.30 \mathrm{E}-05$ & $8.33 \mathrm{E}-05$ & $8.33 \mathrm{E}-05$ & $8.34 \mathrm{E}-05$ \\
\hline$I 137$ & $2.71 \mathrm{E}-05$ & $2.72 \mathrm{E}-05$ & $2.72 \mathrm{E}-05$ & $2.72 \mathrm{E}-05$ & $2.72 \mathrm{E}-05$ \\
\hline 1138 & $5.11 \mathrm{E}-09$ & $5.13 \mathrm{E}-09$ & $5.12 \mathrm{E}-09$ & $5.11 \mathrm{E}-09$ & 5.11E-09 \\
\hline$I 139$ & $1.43 \mathrm{E}-13$ & $1.44 \mathrm{E}-13$ & $1.43 \mathrm{E}-13$ & $1.43 \mathrm{E}-13$ & $1.42 \mathrm{E}-13$ \\
\hline Xe128 & $9.68 \mathrm{E}+00$ & $9.08 \mathrm{E}+00$ & $8.68 \mathrm{E}+00$ & $8.56 \mathrm{E}+00$ & $8.53 \mathrm{E}+00$ \\
\hline Xe130 & $1.28 \mathrm{E}+01$ & $1.06 \mathrm{E}+01$ & $9.15 \mathrm{E}+00$ & $8.70 \mathrm{E}+00$ & $8.59 \mathrm{E}+00$ \\
\hline Xe131 & $6.81 \mathrm{E}+02$ & $6.98 \mathrm{E}+02$ & $7.17 \mathrm{E}+02$ & $7.25 \mathrm{E}+02$ & $7.27 \mathrm{E}+02$ \\
\hline Xe132 & $1.81 \mathrm{E}+03$ & $1.77 \mathrm{E}+03$ & $1.74 \mathrm{E}+03$ & $1.73 \mathrm{E}+03$ & $1.73 \mathrm{E}+03$ \\
\hline Xe133 & $9.90 \mathrm{E}+00$ & $9.90 \mathrm{E}+00$ & $9.90 \mathrm{E}+00$ & $9.90 \mathrm{E}+00$ & $9.90 \mathrm{E}+00$ \\
\hline Xe133M & $5.95 \mathrm{E}-02$ & $5.92 \mathrm{E}-02$ & $5.91 \mathrm{E}-02$ & $5.91 \mathrm{E}-02$ & $5.92 \mathrm{E}-02$ \\
\hline Xe134 & $2.21 \mathrm{E}+03$ & $2.21 \mathrm{E}+03$ & $2.21 \mathrm{E}+03$ & $2.21 \mathrm{E}+03$ & $2.20 \mathrm{E}+03$ \\
\hline Xe135 & $3.00 \mathrm{E}-01$ & $3.84 \mathrm{E}-01$ & $4.65 \mathrm{E}-01$ & $4.96 \mathrm{E}-01$ & $5.04 \mathrm{E}-01$ \\
\hline Xe135M & $3.49 \mathrm{E}-03$ & $3.48 \mathrm{E}-03$ & $3.49 \mathrm{E}-03$ & $3.50 \mathrm{E}-03$ & $3.50 \mathrm{E}-03$ \\
\hline Xe136 & $3.19 \mathrm{E}+03$ & $3.00 \mathrm{E}+03$ & $2.83 \mathrm{E}+03$ & $2.76 \mathrm{E}+03$ & $2.74 \mathrm{E}+03$ \\
\hline Xe137 & $3.96 \mathrm{E}-03$ & $3.97 \mathrm{E}-03$ & $3.97 \mathrm{E}-03$ & $3.97 \mathrm{E}-03$ & $3.97 \mathrm{E}-03$ \\
\hline Xe138 & $1.51 \mathrm{E}-02$ & $1.51 \mathrm{E}-02$ & $1.51 \mathrm{E}-02$ & $1.51 \mathrm{E}-02$ & $1.51 \mathrm{E}-02$ \\
\hline Xe139 & $1.32 \mathrm{E}-04$ & $1.32 \mathrm{E}-04$ & $1.32 \mathrm{E}-04$ & $1.32 \mathrm{E}-04$ & $1.32 \mathrm{E}-04$ \\
\hline Xe140 & $1.95 \mathrm{E}-06$ & $1.96 \mathrm{E}-06$ & $1.95 \mathrm{E}-06$ & $1.95 \mathrm{E}-06$ & $1.94 \mathrm{E}-06$ \\
\hline Xe141 & $1.72 \mathrm{E}-13$ & $1.73 \mathrm{E}-13$ & $1.72 \mathrm{E}-13$ & $1.72 \mathrm{E}-13$ & $1.71 \mathrm{E}-13$ \\
\hline Cs 133 & $1.63 \mathrm{E}+03$ & $1.64 \mathrm{E}+03$ & $1.65 \mathrm{E}+03$ & $1.66 \mathrm{E}+03$ & $1.66 \mathrm{E}+03$ \\
\hline Cs 134 & $2.13 \mathrm{E}+02$ & $2.02 \mathrm{E}+02$ & $1.92 \mathrm{E}+02$ & $1.88 \mathrm{E}+02$ & $1.87 \mathrm{E}+02$ \\
\hline Cs $134 M$ & 7.99E-03 & 7.35E-03 & $6.84 \mathrm{E}-03$ & $6.66 \mathrm{E}-03$ & $6.61 \mathrm{E}-03$ \\
\hline Cs 135 & $9.91 \mathrm{E}+02$ & $1.16 \mathrm{E}+03$ & $1.32 \mathrm{E}+03$ & $1.38 \mathrm{E}+03$ & $1.40 \mathrm{E}+03$ \\
\hline Cs $135 M$ & $2.01 \mathrm{E}-04$ & $2.19 \mathrm{E}-04$ & $2.39 \mathrm{E}-04$ & $2.47 \mathrm{E}-04$ & $2.49 \mathrm{E}-04$ \\
\hline Cs 136 & $1.13 \mathrm{E}+00$ & $1.19 \mathrm{E}+00$ & $1.24 \mathrm{E}+00$ & $1.26 \mathrm{E}+00$ & $1.27 \mathrm{E}+00$ \\
\hline Cs 137 & $1.83 \mathrm{E}+03$ & $1.83 \mathrm{E}+03$ & $1.83 \mathrm{E}+03$ & $1.83 \mathrm{E}+03$ & $1.83 \mathrm{E}+03$ \\
\hline Cs 138 & $4.21 \mathrm{E}-02$ & $4.22 \mathrm{E}-02$ & 4.22E-02 & $4.23 \mathrm{E}-02$ & $4.23 \mathrm{E}-02$ \\
\hline Cs $138 M$ & $1.89 \mathrm{E}-04$ & $1.88 \mathrm{E}-04$ & $1.89 \mathrm{E}-04$ & $1.89 \mathrm{E}-04$ & $1.90 \mathrm{E}-04$ \\
\hline
\end{tabular}




\begin{tabular}{|c|c|c|c|c|c|}
\hline \multicolumn{6}{|c|}{ Isotopic output mass vector } \\
\hline Isotope & $1^{\text {st }}$ Cycle & $2^{\text {nd }}$ Cycle & $5^{\text {th }}$ Cycle & $10^{\text {th }}$ Cycle & $15^{\text {th }}$ Cycle \\
\hline Cs139 & $1.03 \mathrm{E}-02$ & $1.03 \mathrm{E}-02$ & $1.03 \mathrm{E}-02$ & $1.03 \mathrm{E}-02$ & $1.03 \mathrm{E}-02$ \\
\hline Cs 140 & 4.84E-04 & 4.87E-04 & 4.87E-04 & 4.87E-04 & 4.87E-04 \\
\hline Cs 141 & $3.59 \mathrm{E}-05$ & $3.60 \mathrm{E}-05$ & $3.59 \mathrm{E}-05$ & $3.59 \mathrm{E}-05$ & $3.59 \mathrm{E}-05$ \\
\hline Cs 142 & $3.76 \mathrm{E}-13$ & $3.79 \mathrm{E}-13$ & $3.79 \mathrm{E}-13$ & $3.78 \mathrm{E}-13$ & $3.78 \mathrm{E}-13$ \\
\hline Cs 143 & $2.11 \mathrm{E}-13$ & $2.13 \mathrm{E}-13$ & $2.12 \mathrm{E}-13$ & $2.12 \mathrm{E}-13$ & $2.11 \mathrm{E}-13$ \\
\hline Ba134 & $1.02 \mathrm{E}+02$ & $9.78 \mathrm{E}+01$ & $9.33 \mathrm{E}+01$ & $9.15 \mathrm{E}+01$ & $9.10 \mathrm{E}+01$ \\
\hline Ba135 & $9.11 \mathrm{E}-01$ & $8.33 \mathrm{E}-01$ & $7.62 \mathrm{E}-01$ & $7.35 \mathrm{E}-01$ & $7.28 \mathrm{E}-01$ \\
\hline Ba136 & $5.82 \mathrm{E}+01$ & $5.95 \mathrm{E}+01$ & $6.13 \mathrm{E}+01$ & $6.20 \mathrm{E}+01$ & $6.22 \mathrm{E}+01$ \\
\hline Ba137 & $8.45 \mathrm{E}+01$ & $8.43 \mathrm{E}+01$ & $8.43 \mathrm{E}+01$ & $8.42 \mathrm{E}+01$ & $8.42 \mathrm{E}+01$ \\
\hline Ba137M & $2.80 \mathrm{E}-04$ & $2.80 \mathrm{E}-04$ & $2.80 \mathrm{E}-04$ & $2.80 \mathrm{E}-04$ & $2.80 \mathrm{E}-04$ \\
\hline Ba138 & $1.83 \mathrm{E}+03$ & $1.83 \mathrm{E}+03$ & $1.83 \mathrm{E}+03$ & $1.83 \mathrm{E}+03$ & $1.83 \mathrm{E}+03$ \\
\hline Ba139 & $1.00 \mathrm{E}-01$ & $1.01 \mathrm{E}-01$ & $1.01 \mathrm{E}-01$ & $1.01 \mathrm{E}-01$ & $1.01 \mathrm{E}-01$ \\
\hline Bal40 & $2.13 \mathrm{E}+01$ & $2.13 \mathrm{E}+01$ & $2.13 \mathrm{E}+01$ & $2.13 \mathrm{E}+01$ & $2.13 \mathrm{E}+01$ \\
\hline Ba141 & $1.91 \mathrm{E}-02$ & $1.91 \mathrm{E}-02$ & $1.91 \mathrm{E}-02$ & $1.91 \mathrm{E}-02$ & $1.91 \mathrm{E}-02$ \\
\hline Bal42 & $9.69 \mathrm{E}-03$ & $9.69 \mathrm{E}-03$ & $9.69 \mathrm{E}-03$ & $9.69 \mathrm{E}-03$ & $9.69 \mathrm{E}-03$ \\
\hline Bal43 & 4.64E-06 & 4.65E-06 & 4.64E-06 & 4.63E-06 & 4.63E-06 \\
\hline Bal44 & $9.80 \mathrm{E}-07$ & $9.82 \mathrm{E}-07$ & $9.79 \mathrm{E}-07$ & $9.77 \mathrm{E}-07$ & $9.77 \mathrm{E}-07$ \\
\hline Bal45 & $5.62 \mathrm{E}-11$ & $5.65 \mathrm{E}-11$ & $5.64 \mathrm{E}-11$ & $5.63 \mathrm{E}-11$ & $5.62 \mathrm{E}-11$ \\
\hline Ba146 & $1.38 \mathrm{E}-13$ & $1.38 \mathrm{E}-13$ & $1.38 \mathrm{E}-13$ & $1.37 \mathrm{E}-13$ & $1.37 \mathrm{E}-13$ \\
\hline La138 & $3.19 \mathrm{E}-02$ & $3.20 \mathrm{E}-02$ & $3.24 \mathrm{E}-02$ & $3.26 \mathrm{E}-02$ & $3.26 \mathrm{E}-02$ \\
\hline La139 & $1.70 \mathrm{E}+03$ & $1.70 \mathrm{E}+03$ & $1.71 \mathrm{E}+03$ & $1.71 \mathrm{E}+03$ & $1.71 \mathrm{E}+03$ \\
\hline La140 & $3.00 \mathrm{E}+00$ & $2.99 \mathrm{E}+00$ & $2.98 \mathrm{E}+00$ & $2.98 \mathrm{E}+00$ & $2.98 \mathrm{E}+00$ \\
\hline La141 & $2.57 \mathrm{E}-01$ & $2.57 \mathrm{E}-01$ & $2.57 \mathrm{E}-01$ & $2.57 \mathrm{E}-01$ & $2.57 \mathrm{E}-01$ \\
\hline La142 & $9.43 \mathrm{E}-02$ & $9.44 \mathrm{E}-02$ & $9.44 \mathrm{E}-02$ & $9.44 \mathrm{E}-02$ & $9.44 \mathrm{E}-02$ \\
\hline La143 & $1.31 \mathrm{E}-02$ & $1.31 \mathrm{E}-02$ & $1.31 \mathrm{E}-02$ & $1.31 \mathrm{E}-02$ & $1.31 \mathrm{E}-02$ \\
\hline La144 & $1.91 \mathrm{E}-04$ & $1.91 \mathrm{E}-04$ & $1.91 \mathrm{E}-04$ & $1.90 \mathrm{E}-04$ & $1.90 \mathrm{E}-04$ \\
\hline La145 & 2.99E-05 & 2.99E-05 & $2.99 \mathrm{E}-05$ & $2.99 \mathrm{E}-05$ & $2.99 \mathrm{E}-05$ \\
\hline La146 & 5.19E-09 & $5.22 \mathrm{E}-09$ & $5.21 \mathrm{E}-09$ & $5.20 \mathrm{E}-09$ & 5.19E-09 \\
\hline La147 & $1.66 \mathrm{E}-11$ & $1.68 \mathrm{E}-11$ & $1.69 \mathrm{E}-11$ & $1.68 \mathrm{E}-11$ & $1.68 \mathrm{E}-11$ \\
\hline La148 & $5.29 \mathrm{E}-14$ & $5.39 \mathrm{E}-14$ & $5.41 \mathrm{E}-14$ & $5.40 \mathrm{E}-14$ & $5.39 \mathrm{E}-14$ \\
\hline $\mathrm{Ce} 140$ & $1.72 \mathrm{E}+03$ & $1.72 \mathrm{E}+03$ & $1.72 \mathrm{E}+03$ & $1.72 \mathrm{E}+03$ & $1.72 \mathrm{E}+03$ \\
\hline Ce141 & $5.09 \mathrm{E}+01$ & $5.09 \mathrm{E}+01$ & $5.09 \mathrm{E}+01$ & $5.09 \mathrm{E}+01$ & $5.09 \mathrm{E}+01$ \\
\hline $\mathrm{Ce} 142$ & $1.51 \mathrm{E}+03$ & $1.50 \mathrm{E}+03$ & $1.50 \mathrm{E}+03$ & $1.49 \mathrm{E}+03$ & $1.49 \mathrm{E}+03$ \\
\hline $\mathrm{Ce} 143$ & $1.93 \mathrm{E}+00$ & $1.93 \mathrm{E}+00$ & $1.93 \mathrm{E}+00$ & $1.93 \mathrm{E}+00$ & $1.93 \mathrm{E}+00$ \\
\hline $\mathrm{Ce} 144$ & $3.45 \mathrm{E}+02$ & $3.45 \mathrm{E}+02$ & $3.44 \mathrm{E}+02$ & $3.44 \mathrm{E}+02$ & $3.44 \mathrm{E}+02$ \\
\hline $\mathrm{Ce} 145$ & $1.73 \mathrm{E}-03$ & $1.73 \mathrm{E}-03$ & $1.73 \mathrm{E}-03$ & $1.73 \mathrm{E}-03$ & $1.73 \mathrm{E}-03$ \\
\hline $\mathrm{Ce} 146$ & 7.29E-03 & $7.31 \mathrm{E}-03$ & 7.32E-03 & 7.32E-03 & 7.31E-03 \\
\hline
\end{tabular}




\begin{tabular}{|c|c|c|c|c|c|}
\hline \multicolumn{6}{|c|}{ Isotopic output mass vector } \\
\hline Isotope & $1^{\text {st }}$ Cycle & $2^{\text {nd }}$ Cycle & $5^{\text {th }}$ Cycle & $10^{\text {th }}$ Cycle & $15^{\text {th }}$ Cycle \\
\hline Cel47 & $1.62 \mathrm{E}-04$ & $1.63 \mathrm{E}-04$ & $1.64 \mathrm{E}-04$ & $1.64 \mathrm{E}-04$ & $1.64 \mathrm{E}-04$ \\
\hline $\mathrm{Ce} 148$ & $1.05 \mathrm{E}-04$ & $1.06 \mathrm{E}-04$ & $1.06 \mathrm{E}-04$ & $1.06 \mathrm{E}-04$ & $1.06 \mathrm{E}-04$ \\
\hline Cel49 & $5.06 \mathrm{E}-10$ & $5.13 \mathrm{E}-10$ & $5.15 \mathrm{E}-10$ & $5.15 \mathrm{E}-10$ & $5.15 \mathrm{E}-10$ \\
\hline Ce150 & $7.10 \mathrm{E}-12$ & $7.20 \mathrm{E}-12$ & $7.23 \mathrm{E}-12$ & $7.23 \mathrm{E}-12$ & $7.22 \mathrm{E}-12$ \\
\hline $\operatorname{Pr} 141$ & $1.50 \mathrm{E}+03$ & $1.49 \mathrm{E}+03$ & $1.49 \mathrm{E}+03$ & $1.49 \mathrm{E}+03$ & $1.49 \mathrm{E}+03$ \\
\hline $\operatorname{Pr} 142$ & $5.02 \mathrm{E}-02$ & $4.18 \mathrm{E}-02$ & $3.63 \mathrm{E}-02$ & $3.46 \mathrm{E}-02$ & $3.41 \mathrm{E}-02$ \\
\hline $\operatorname{Pr} 143$ & $1.84 \mathrm{E}+01$ & $1.84 \mathrm{E}+01$ & $1.83 \mathrm{E}+01$ & $1.83 \mathrm{E}+01$ & $1.83 \mathrm{E}+01$ \\
\hline $\operatorname{Pr} 144$ & $1.46 \mathrm{E}-02$ & $1.46 \mathrm{E}-02$ & $1.46 \mathrm{E}-02$ & $1.45 \mathrm{E}-02$ & $1.45 \mathrm{E}-02$ \\
\hline $\operatorname{Pr} 145$ & $2.47 \mathrm{E}-01$ & $2.47 \mathrm{E}-01$ & $2.47 \mathrm{E}-01$ & $2.47 \mathrm{E}-01$ & $2.47 \mathrm{E}-01$ \\
\hline $\operatorname{Pr} 146$ & $1.39 \mathrm{E}-02$ & $1.39 \mathrm{E}-02$ & $1.39 \mathrm{E}-02$ & $1.39 \mathrm{E}-02$ & $1.39 \mathrm{E}-02$ \\
\hline $\operatorname{Pr} 147$ & $6.12 \mathrm{E}-03$ & $6.14 \mathrm{E}-03$ & $6.15 \mathrm{E}-03$ & $6.15 \mathrm{E}-03$ & $6.15 \mathrm{E}-03$ \\
\hline $\operatorname{Pr} 148$ & $6.66 \mathrm{E}-04$ & $6.71 \mathrm{E}-04$ & $6.74 \mathrm{E}-04$ & $6.74 \mathrm{E}-04$ & $6.74 \mathrm{E}-04$ \\
\hline $\operatorname{Pr} 149$ & $4.40 \mathrm{E}-04$ & 4.43E-04 & $4.45 \mathrm{E}-04$ & $4.45 \mathrm{E}-04$ & $4.45 \mathrm{E}-04$ \\
\hline $\operatorname{Pr} 150$ & 5.37E-09 & $5.44 \mathrm{E}-09$ & $5.46 \mathrm{E}-09$ & $5.46 \mathrm{E}-09$ & $5.46 \mathrm{E}-09$ \\
\hline $\operatorname{Pr} 151$ & $2.16 \mathrm{E}-06$ & $2.19 \mathrm{E}-06$ & $2.20 \mathrm{E}-06$ & $2.20 \mathrm{E}-06$ & $2.20 \mathrm{E}-06$ \\
\hline $\operatorname{Pr} 152$ & $1.11 \mathrm{E}-12$ & $1.14 \mathrm{E}-12$ & $1.15 \mathrm{E}-12$ & $1.15 \mathrm{E}-12$ & $1.15 \mathrm{E}-12$ \\
\hline $\operatorname{Pr} 153$ & 4.74E-12 & $4.87 \mathrm{E}-12$ & $4.92 \mathrm{E}-12$ & $4.92 \mathrm{E}-12$ & $4.92 \mathrm{E}-12$ \\
\hline$N d 142$ & $2.61 \mathrm{E}+01$ & $2.26 \mathrm{E}+01$ & $2.02 \mathrm{E}+01$ & $1.94 \mathrm{E}+01$ & $1.92 \mathrm{E}+01$ \\
\hline$N d 143$ & $1.08 \mathrm{E}+03$ & $1.14 \mathrm{E}+03$ & $1.18 \mathrm{E}+03$ & $1.19 \mathrm{E}+03$ & $1.20 \mathrm{E}+03$ \\
\hline $\mathrm{Nd144}$ & $1.18 \mathrm{E}+03$ & $1.11 \mathrm{E}+03$ & $1.07 \mathrm{E}+03$ & $1.05 \mathrm{E}+03$ & $1.05 \mathrm{E}+03$ \\
\hline $\mathrm{Nd145}$ & $8.50 \mathrm{E}+02$ & $8.61 \mathrm{E}+02$ & $8.69 \mathrm{E}+02$ & $8.72 \mathrm{E}+02$ & $8.73 \mathrm{E}+02$ \\
\hline$N d 146$ & $9.45 \mathrm{E}+02$ & $9.35 \mathrm{E}+02$ & $9.26 \mathrm{E}+02$ & $9.22 \mathrm{E}+02$ & $9.21 \mathrm{E}+02$ \\
\hline$N d 147$ & $7.40 \mathrm{E}+00$ & $7.43 \mathrm{E}+00$ & $7.45 \mathrm{E}+00$ & $7.45 \mathrm{E}+00$ & $7.45 \mathrm{E}+00$ \\
\hline $\mathrm{Nd148}$ & $5.44 \mathrm{E}+02$ & $5.48 \mathrm{E}+02$ & $5.50 \mathrm{E}+02$ & $5.50 \mathrm{E}+02$ & $5.50 \mathrm{E}+02$ \\
\hline$N d 149$ & $3.13 \mathrm{E}-02$ & $3.14 \mathrm{E}-02$ & $3.15 \mathrm{E}-02$ & $3.15 \mathrm{E}-02$ & $3.15 \mathrm{E}-02$ \\
\hline$N d 150$ & $3.17 \mathrm{E}+02$ & $3.23 \mathrm{E}+02$ & $3.25 \mathrm{E}+02$ & $3.26 \mathrm{E}+02$ & $3.26 \mathrm{E}+02$ \\
\hline$N d 151$ & $2.10 \mathrm{E}-03$ & $2.12 \mathrm{E}-03$ & $2.13 \mathrm{E}-03$ & $2.13 \mathrm{E}-03$ & $2.13 \mathrm{E}-03$ \\
\hline$N d 152$ & $1.37 \mathrm{E}-03$ & $1.39 \mathrm{E}-03$ & $1.40 \mathrm{E}-03$ & $1.41 \mathrm{E}-03$ & $1.41 \mathrm{E}-03$ \\
\hline$N d 153$ & $7.74 \mathrm{E}-06$ & 7.91E-06 & $8.00 \mathrm{E}-06$ & $8.02 \mathrm{E}-06$ & $8.03 \mathrm{E}-06$ \\
\hline Nd154 & $2.43 \mathrm{E}-06$ & $2.48 \mathrm{E}-06$ & $2.51 \mathrm{E}-06$ & $2.52 \mathrm{E}-06$ & $2.52 \mathrm{E}-06$ \\
\hline Nd155 & 5.92E-09 & $6.11 \mathrm{E}-09$ & $6.20 \mathrm{E}-09$ & $6.22 \mathrm{E}-09$ & $6.22 \mathrm{E}-09$ \\
\hline Pm147 & $2.14 \mathrm{E}+02$ & $2.24 \mathrm{E}+02$ & $2.33 \mathrm{E}+02$ & $2.36 \mathrm{E}+02$ & $2.37 \mathrm{E}+02$ \\
\hline Pm148 & $1.23 \mathrm{E}+00$ & $1.18 \mathrm{E}+00$ & $1.13 \mathrm{E}+00$ & $1.11 \mathrm{E}+00$ & $1.10 \mathrm{E}+00$ \\
\hline Pm148M & $2.50 \mathrm{E}+00$ & $3.03 \mathrm{E}+00$ & $3.52 \mathrm{E}+00$ & $3.71 \mathrm{E}+00$ & $3.75 \mathrm{E}+00$ \\
\hline Pm149 & $1.31 \mathrm{E}+00$ & $1.25 \mathrm{E}+00$ & $1.20 \mathrm{E}+00$ & $1.18 \mathrm{E}+00$ & $1.18 \mathrm{E}+00$ \\
\hline Pm150 & $5.68 \mathrm{E}-04$ & $4.30 \mathrm{E}-04$ & $3.40 \mathrm{E}-04$ & $3.12 \mathrm{E}-04$ & $3.05 \mathrm{E}-04$ \\
\hline Pm151 & $3.06 \mathrm{E}-01$ & $3.09 \mathrm{E}-01$ & $3.11 \mathrm{E}-01$ & $3.12 \mathrm{E}-01$ & $3.12 \mathrm{E}-01$ \\
\hline
\end{tabular}




\begin{tabular}{|c|c|c|c|c|c|}
\hline \multicolumn{6}{|c|}{ Isotopic output mass vector } \\
\hline Isotope & $1^{\text {st }}$ Cycle & $2^{\text {nd }}$ Cycle & $5^{\text {th }}$ Cycle & $10^{\text {th }}$ Cycle & $15^{\text {th }}$ Cycle \\
\hline Pm152 & $5.36 \mathrm{E}-04$ & $5.44 \mathrm{E}-04$ & $5.49 \mathrm{E}-04$ & $5.50 \mathrm{E}-04$ & $5.51 \mathrm{E}-04$ \\
\hline$P m 152 M$ & $2.23 \mathrm{E}-05$ & $2.22 \mathrm{E}-05$ & $2.23 \mathrm{E}-05$ & $2.24 \mathrm{E}-05$ & $2.25 \mathrm{E}-05$ \\
\hline Pm153 & 4.38E-04 & 4.47E-04 & $4.52 \mathrm{E}-04$ & $4.54 \mathrm{E}-04$ & 4.54E-04 \\
\hline Pm154 & $1.19 \mathrm{E}-04$ & $1.21 \mathrm{E}-04$ & $1.23 \mathrm{E}-04$ & $1.23 \mathrm{E}-04$ & $1.23 \mathrm{E}-04$ \\
\hline Pm154M & $7.93 \mathrm{E}-06$ & 8.04E-06 & $8.14 \mathrm{E}-06$ & $8.19 \mathrm{E}-06$ & $8.21 \mathrm{E}-06$ \\
\hline Pm155 & $7.76 \mathrm{E}-06$ & $7.97 \mathrm{E}-06$ & $8.10 \mathrm{E}-06$ & $8.14 \mathrm{E}-06$ & $8.14 \mathrm{E}-06$ \\
\hline Pm156 & $1.36 \mathrm{E}-06$ & $1.40 \mathrm{E}-06$ & $1.42 \mathrm{E}-06$ & $1.42 \mathrm{E}-06$ & $1.43 \mathrm{E}-06$ \\
\hline Pm157 & $1.11 \mathrm{E}-08$ & $1.15 \mathrm{E}-08$ & $1.17 \mathrm{E}-08$ & $1.18 \mathrm{E}-08$ & $1.18 \mathrm{E}-08$ \\
\hline $\operatorname{Sm} 147$ & $1.10 \mathrm{E}+02$ & $1.16 \mathrm{E}+02$ & $1.21 \mathrm{E}+02$ & $1.23 \mathrm{E}+02$ & $1.23 \mathrm{E}+02$ \\
\hline $\operatorname{Sm} 148$ & $2.33 \mathrm{E}+02$ & $2.35 \mathrm{E}+02$ & $2.35 \mathrm{E}+02$ & $2.34 \mathrm{E}+02$ & $2.34 \mathrm{E}+02$ \\
\hline $\operatorname{Sm} 149$ & $3.99 \mathrm{E}+00$ & $5.88 \mathrm{E}+00$ & $8.40 \mathrm{E}+00$ & $9.66 \mathrm{E}+00$ & $1.00 \mathrm{E}+01$ \\
\hline$S m 150$ & $4.49 \mathrm{E}+02$ & $4.45 \mathrm{E}+02$ & $4.38 \mathrm{E}+02$ & $4.35 \mathrm{E}+02$ & $4.34 \mathrm{E}+02$ \\
\hline$S m 151$ & $2.41 \mathrm{E}+01$ & $3.28 \mathrm{E}+01$ & $4.34 \mathrm{E}+01$ & $4.82 \mathrm{E}+01$ & $4.96 \mathrm{E}+01$ \\
\hline Sm152 & $1.69 \mathrm{E}+02$ & $1.76 \mathrm{E}+02$ & $1.82 \mathrm{E}+02$ & $1.84 \mathrm{E}+02$ & $1.85 \mathrm{E}+02$ \\
\hline Sm153 & $1.31 \mathrm{E}+00$ & $1.24 \mathrm{E}+00$ & $1.18 \mathrm{E}+00$ & $1.16 \mathrm{E}+00$ & $1.15 \mathrm{E}+00$ \\
\hline Sm154 & $8.79 \mathrm{E}+01$ & $9.04 \mathrm{E}+01$ & $9.18 \mathrm{E}+01$ & $9.21 \mathrm{E}+01$ & $9.22 \mathrm{E}+01$ \\
\hline Sm155 & $9.81 \mathrm{E}-04$ & $9.98 \mathrm{E}-04$ & $1.01 \mathrm{E}-03$ & $1.01 \mathrm{E}-03$ & $1.01 \mathrm{E}-03$ \\
\hline Sm156 & $1.67 \mathrm{E}-02$ & $1.71 \mathrm{E}-02$ & $1.74 \mathrm{E}-02$ & $1.75 \mathrm{E}-02$ & $1.75 \mathrm{E}-02$ \\
\hline Sm157 & $1.40 \mathrm{E}-04$ & $1.45 \mathrm{E}-04$ & $1.48 \mathrm{E}-04$ & $1.49 \mathrm{E}-04$ & $1.49 \mathrm{E}-04$ \\
\hline Sm158 & $5.01 \mathrm{E}-05$ & $5.23 \mathrm{E}-05$ & $5.36 \mathrm{E}-05$ & $5.39 \mathrm{E}-05$ & $5.40 \mathrm{E}-05$ \\
\hline Eu151 & $4.26 \mathrm{E}-02$ & $7.87 \mathrm{E}-02$ & $1.36 \mathrm{E}-01$ & $1.67 \mathrm{E}-01$ & $1.77 \mathrm{E}-01$ \\
\hline Eu152 & $3.83 \mathrm{E}-02$ & $7.45 \mathrm{E}-02$ & $1.30 \mathrm{E}-01$ & $1.59 \mathrm{E}-01$ & $1.67 \mathrm{E}-01$ \\
\hline Eu153 & $2.26 \mathrm{E}+02$ & $2.30 \mathrm{E}+02$ & $2.30 \mathrm{E}+02$ & $2.30 \mathrm{E}+02$ & $2.30 \mathrm{E}+02$ \\
\hline Eu154 & $6.58 \mathrm{E}+01$ & $7.23 \mathrm{E}+01$ & $7.57 \mathrm{E}+01$ & $7.62 \mathrm{E}+01$ & $7.62 \mathrm{E}+01$ \\
\hline Eu155 & $1.78 \mathrm{E}+01$ & $1.79 \mathrm{E}+01$ & $1.78 \mathrm{E}+01$ & $1.77 \mathrm{E}+01$ & $1.77 \mathrm{E}+01$ \\
\hline Eu156 & $6.56 \mathrm{E}+00$ & $5.47 \mathrm{E}+00$ & $4.56 \mathrm{E}+00$ & $4.24 \mathrm{E}+00$ & $4.16 \mathrm{E}+00$ \\
\hline Eu157 & $2.45 \mathrm{E}-02$ & $2.35 \mathrm{E}-02$ & $2.27 \mathrm{E}-02$ & $2.24 \mathrm{E}-02$ & $2.23 \mathrm{E}-02$ \\
\hline Eu158 & $5.55 \mathrm{E}-04$ & $5.79 \mathrm{E}-04$ & $5.93 \mathrm{E}-04$ & $5.97 \mathrm{E}-04$ & $5.97 \mathrm{E}-04$ \\
\hline Eu159 & $1.07 \mathrm{E}-04$ & $1.13 \mathrm{E}-04$ & $1.16 \mathrm{E}-04$ & $1.17 \mathrm{E}-04$ & $1.17 \mathrm{E}-04$ \\
\hline Eu160 & $4.83 \mathrm{E}-07$ & $5.16 \mathrm{E}-07$ & $5.34 \mathrm{E}-07$ & $5.39 \mathrm{E}-07$ & $5.40 \mathrm{E}-07$ \\
\hline Gd152 & $9.36 \mathrm{E}-02$ & $1.33 \mathrm{E}-01$ & $1.74 \mathrm{E}-01$ & $1.90 \mathrm{E}-01$ & $1.95 \mathrm{E}-01$ \\
\hline Gd154 & $7.39 \mathrm{E}+00$ & $7.86 \mathrm{E}+00$ & $8.00 \mathrm{E}+00$ & $7.97 \mathrm{E}+00$ & $7.95 \mathrm{E}+00$ \\
\hline Gd155 & $2.53 \mathrm{E}-01$ & $3.78 \mathrm{E}-01$ & $5.37 \mathrm{E}-01$ & $6.13 \mathrm{E}-01$ & $6.35 \mathrm{E}-01$ \\
\hline Gd156 & $1.84 \mathrm{E}+02$ & $1.64 \mathrm{E}+02$ & $1.48 \mathrm{E}+02$ & $1.42 \mathrm{E}+02$ & $1.40 \mathrm{E}+02$ \\
\hline Gd157 & $2.99 \mathrm{E}-01$ & $4.25 \mathrm{E}-01$ & $5.96 \mathrm{E}-01$ & $6.82 \mathrm{E}-01$ & 7.07E-01 \\
\hline Gd158 & $5.13 \mathrm{E}+01$ & $5.23 \mathrm{E}+01$ & $5.25 \mathrm{E}+01$ & $5.25 \mathrm{E}+01$ & $5.24 \mathrm{E}+01$ \\
\hline Gd159 & $1.01 \mathrm{E}-02$ & $1.03 \mathrm{E}-02$ & $1.04 \mathrm{E}-02$ & $1.04 \mathrm{E}-02$ & $1.04 \mathrm{E}-02$ \\
\hline
\end{tabular}




\begin{tabular}{|c|c|c|c|c|c|}
\hline \multicolumn{7}{|c|}{ Isotopic output mass vector } \\
\hline Isotope & $\mathbf{1}^{\text {st }} \mathbf{C y c l e}$ & $\mathbf{2}^{\text {nd }} \mathbf{C y c l e}$ & $\mathbf{5}^{\text {th }}$ Cycle & $\mathbf{1 0}^{\text {th }}$ Cycle & $\mathbf{1 5}^{\text {th }}$ Cycle \\
\hline Gd160 & $3.50 \mathrm{E}+00$ & $3.81 \mathrm{E}+00$ & $4.00 \mathrm{E}+00$ & $4.06 \mathrm{E}+00$ & $4.07 \mathrm{E}+00$ \\
\hline$G d 161$ & $4.27 \mathrm{E}-06$ & $4.64 \mathrm{E}-06$ & $4.85 \mathrm{E}-06$ & $4.91 \mathrm{E}-06$ & $4.93 \mathrm{E}-06$ \\
\hline$T b 159$ & $7.49 \mathrm{E}+00$ & $8.09 \mathrm{E}+00$ & $8.45 \mathrm{E}+00$ & $8.56 \mathrm{E}+00$ & $8.58 \mathrm{E}+00$ \\
\hline$T b 160$ & $2.32 \mathrm{E}-01$ & $2.40 \mathrm{E}-01$ & $2.40 \mathrm{E}-01$ & $2.38 \mathrm{E}-01$ & $2.38 \mathrm{E}-01$ \\
\hline Tb161 & $1.77 \mathrm{E}-02$ & $1.83 \mathrm{E}-02$ & $1.85 \mathrm{E}-02$ & $1.85 \mathrm{E}-02$ & $1.85 \mathrm{E}-02$ \\
\hline$D y 160$ & $8.35 \mathrm{E}-01$ & $9.09 \mathrm{E}-01$ & $9.45 \mathrm{E}-01$ & $9.53 \mathrm{E}-01$ & $9.54 \mathrm{E}-01$ \\
\hline$D y 161$ & $1.22 \mathrm{E}+00$ & $1.39 \mathrm{E}+00$ & $1.51 \mathrm{E}+00$ & $1.55 \mathrm{E}+00$ & $1.56 \mathrm{E}+00$ \\
\hline$D y 162$ & $8.37 \mathrm{E}-01$ & $8.67 \mathrm{E}-01$ & $8.79 \mathrm{E}-01$ & $8.83 \mathrm{E}-01$ & $8.83 \mathrm{E}-01$ \\
\hline$D y 163$ & $7.33 \mathrm{E}-01$ & $7.37 \mathrm{E}-01$ & $7.36 \mathrm{E}-01$ & $7.36 \mathrm{E}-01$ & $7.36 \mathrm{E}-01$ \\
\hline$D y 164$ & $2.65 \mathrm{E}-01$ & $3.02 \mathrm{E}-01$ & $3.28 \mathrm{E}-01$ & $3.37 \mathrm{E}-01$ & $3.39 \mathrm{E}-01$ \\
\hline$D y 166$ & $8.77 \mathrm{E}-05$ & $1.03 \mathrm{E}-04$ & $1.12 \mathrm{E}-04$ & $1.15 \mathrm{E}-04$ & $1.16 \mathrm{E}-04$ \\
\hline$H o 165$ & $3.05 \mathrm{E}-01$ & $2.46 \mathrm{E}-01$ & $2.00 \mathrm{E}-01$ & $1.85 \mathrm{E}-01$ & $1.81 \mathrm{E}-01$ \\
\hline$E r 166$ & $1.01 \mathrm{E}-01$ & $8.48 \mathrm{E}-02$ & $7.40 \mathrm{E}-02$ & $7.10 \mathrm{E}-02$ & $7.03 \mathrm{E}-02$ \\
\hline$E r 167$ & $5.37 \mathrm{E}-03$ & $5.40 \mathrm{E}-03$ & $5.63 \mathrm{E}-03$ & $5.80 \mathrm{E}-03$ & $5.85 \mathrm{E}-03$ \\
\hline$E r 168$ & $1.59 \mathrm{E}-02$ & $1.55 \mathrm{E}-02$ & $1.53 \mathrm{E}-02$ & $1.53 \mathrm{E}-02$ & $1.53 \mathrm{E}-02$ \\
\hline
\end{tabular}




\section{Appendix 3: Mid-cycle 1-group cross sections of most important} actinides

\begin{tabular}{|c|c|c|c|c|c|}
\hline Isotope & $1^{\text {st }}$ Cycle & $2^{\text {nd }}$ Cycle & $5^{\text {th }}$ Cycle & $10^{\text {th }}$ Cycle & $15^{\text {th }}$ Cycle \\
\hline IDs & \multicolumn{5}{|c|}{ Weighted using the total neutron flux } \\
\hline U232 & 13.03000 & 12.58000 & 12.16000 & 11.99000 & 11.95000 \\
\hline$U 233$ & 36.62000 & 32.72000 & 29.69000 & 28.60000 & 28.31000 \\
\hline$U 234$ & 0.53730 & 0.54750 & 0.55640 & 0.55990 & 0.56090 \\
\hline$U 235$ & 19.49000 & 16.46000 & 14.19000 & 13.41000 & 13.21000 \\
\hline$U 236$ & 0.34140 & 0.34410 & 0.34560 & 0.34620 & 0.34630 \\
\hline$U 238$ & 0.11510 & 0.11740 & 0.11950 & 0.12040 & 0.12060 \\
\hline Np237 & 0.59220 & 0.60380 & 0.61380 & 0.61780 & 0.61890 \\
\hline Pu238 & 1.99100 & 1.94600 & 1.90700 & 1.89200 & 1.88800 \\
\hline Pu239 & 37.21000 & 30.57000 & 25.24000 & 23.35000 & 22.85000 \\
\hline Pu240 & 0.63200 & 0.63350 & 0.64140 & 0.64480 & 0.64580 \\
\hline Pu241 & 44.62000 & 37.34000 & 31.66000 & 29.68000 & 29.16000 \\
\hline Pu242 & 0.47570 & 0.48550 & 0.49370 & 0.49700 & 0.49790 \\
\hline Pu244 & 0.39960 & 0.40710 & 0.41380 & 0.41660 & 0.41730 \\
\hline Am241 & 0.88110 & 0.84230 & 0.81370 & 0.80380 & 0.80120 \\
\hline$A m 242 m$ & 225.30000 & 178.10000 & 142.40000 & 130.10000 & 126.90000 \\
\hline Am243 & 0.48200 & 0.48670 & 0.49130 & 0.49320 & 0.49380 \\
\hline $\mathrm{Cm} 242$ & 0.46030 & 0.45180 & 0.44670 & 0.44540 & 0.44520 \\
\hline $\mathrm{Cm} 243$ & 54.19000 & 48.18000 & 43.39000 & 41.61000 & 41.14000 \\
\hline $\mathrm{Cm} 244$ & 0.85870 & 0.86690 & 0.87310 & 0.87550 & 0.87620 \\
\hline $\mathrm{Cm} 245$ & 56.16000 & 46.60000 & 39.38000 & 36.88000 & 36.23000 \\
\hline $\mathrm{Cm} 246$ & 0.63800 & 0.64370 & 0.64820 & 0.65000 & 0.65060 \\
\hline Cm247 & 31.66000 & 27.41000 & 24.71000 & 23.75000 & 23.51000 \\
\hline Cm248 & 0.79750 & 0.80700 & 0.81290 & 0.81490 & 0.81540 \\
\hline $\mathrm{Cm} 250$ & 0.51710 & 0.52760 & 0.53670 & 0.54040 & 0.54140 \\
\hline$B k 249$ & 1.23600 & 1.10700 & 1.00800 & 0.97290 & 0.96390 \\
\hline$C f 249$ & 99.24000 & 83.99000 & 73.21000 & 69.39000 & 68.41000 \\
\hline$C f 250$ & 14.16000 & 10.94000 & 9.61600 & 9.19400 & 9.08800 \\
\hline$C f 251$ & 281.40000 & 233.90000 & 200.10000 & 188.20000 & 185.10000 \\
\hline$C f 252$ & 4.89000 & 4.65100 & 4.46400 & 4.39500 & 4.37700 \\
\hline
\end{tabular}




\begin{tabular}{|c|c|c|c|c|c|}
\hline Isotope & $1^{\text {st }}$ Cycle & $2^{\text {nd }}$ Cycle & $5^{\text {th }}$ Cycle & $10^{\text {th }}$ Cycle & $15^{\text {th }}$ Cycle \\
\hline IDs & \multicolumn{5}{|c|}{ Weighted using the total neutron flux } \\
\hline$U 232$ & 9.93600 & 9.47600 & 9.05300 & 8.89100 & 8.84800 \\
\hline$U 233$ & 5.13200 & 4.70300 & 4.34400 & 4.20800 & 4.17200 \\
\hline$U 234$ & 18.25000 & 17.52000 & 16.80000 & 16.51000 & 16.44000 \\
\hline$U 235$ & 5.77700 & 5.23500 & 4.80800 & 4.65400 & 4.61400 \\
\hline$U 236$ & 8.78900 & 8.62200 & 8.40400 & 8.31100 & 8.28600 \\
\hline$U 238$ & 0.86360 & 0.85360 & 0.84440 & 0.84120 & 0.84040 \\
\hline Np237 & 25.32000 & 23.07000 & 21.20000 & 20.48000 & 20.29000 \\
\hline Pu238 & 13.80000 & 11.52000 & 9.67000 & 9.01600 & 8.84600 \\
\hline Pu239 & 20.28000 & 16.77000 & 13.90000 & 12.86000 & 12.59000 \\
\hline Pu240 & 96.03000 & 43.53000 & 31.49000 & 28.26000 & 27.52000 \\
\hline Pu241 & 15.51000 & 12.82000 & 10.73000 & 10.00000 & 9.81000 \\
\hline Pu242 & 35.01000 & 24.37000 & 15.76000 & 12.99000 & 12.27000 \\
\hline Pu244 & 3.17500 & 3.18300 & 3.16500 & 3.15200 & 3.14800 \\
\hline Am241 & 57.23000 & 48.59000 & 41.91000 & 39.47000 & 38.83000 \\
\hline Am242m & 42.33000 & 33.36000 & 26.52000 & 24.16000 & 23.55000 \\
\hline Am243 & 49.34000 & 44.65000 & 40.91000 & 39.46000 & 39.08000 \\
\hline $\mathrm{Cm} 242$ & 3.64700 & 3.53400 & 3.42200 & 3.37600 & 3.36400 \\
\hline $\mathrm{Cm} 243$ & 8.22500 & 7.32300 & 6.60000 & 6.33400 & 6.26400 \\
\hline $\mathrm{Cm} 244$ & 16.09000 & 15.78000 & 15.27000 & 15.02000 & 14.95000 \\
\hline $\mathrm{Cm} 245$ & 8.34800 & 6.92000 & 5.82900 & 5.45300 & 5.35500 \\
\hline $\mathrm{Cm} 246$ & 3.41600 & 3.34000 & 3.25200 & 3.21700 & 3.20700 \\
\hline $\mathrm{Cm} 247$ & 15.69000 & 13.38000 & 11.92000 & 11.40000 & 11.26000 \\
\hline $\mathrm{Cm} 248$ & 6.65400 & 6.59000 & 6.45700 & 6.39100 & 6.37100 \\
\hline $\mathrm{Cm} 250$ & 50.64000 & 49.42000 & 47.97000 & 47.36000 & 47.19000 \\
\hline$B k 249$ & 157.10000 & 132.70000 & 113.80000 & 107.10000 & 105.40000 \\
\hline$C f 249$ & 34.82000 & 29.50000 & 25.78000 & 24.45000 & 24.11000 \\
\hline$C f 250$ & 342.00000 & 292.80000 & 258.80000 & 246.30000 & 243.00000 \\
\hline$C f 251$ & 121.70000 & 100.30000 & 84.83000 & 79.42000 & 78.02000 \\
\hline$C f 252$ & 2.02900 & 1.91500 & 1.81900 & 1.78300 & 1.77400 \\
\hline
\end{tabular}

